\title{
Study of the $\Lambda_{b} \rightarrow N^{*} \ell^{+} \ell^{-}$decay in light cone sum rules
}

\author{
T. M. Aliev ${ }^{1, \mathrm{a}}$, T. Barakat $^{2, \mathrm{~b}}$, M. Savci ${ }^{1, \mathrm{c}}$ \\ ${ }_{1}^{1}$ Physics Department, Middle East Technical University, 06531 Ankara, Turkey \\ 2 Physics Department, King Saud University, Riyadh 11451, Saudi Arabia
}

Received: 18 January 2019 / Accepted: 15 April 2019 / Published online: 3 May 2019

(C) The Author(s) 2019

\begin{abstract}
The form factors of the $\Lambda_{b} \rightarrow N^{*} \ell^{+} \ell^{-}$decay are calculated in the framework of the light cone QCD sum rules. In the calculations the contribution of the negative parity $\Lambda_{b}^{*}$ baryon is eliminated by constructing the sum rules for different Lorentz structures. Furthermore the branching ratio of the semileptonic $\Lambda_{b} \rightarrow N^{*} \ell^{+} \ell^{-}$decay is calculated. The numerical study for the branching ratio of the $\Lambda_{b} \rightarrow N^{*} \ell^{+} \ell^{-}$decay indicates that it is quite large and could be measurable at future planned experiments to be conducted at LHCb.
\end{abstract}

\section{Introduction}

Lately, exciting experimental results have been obtained in study of the rare decays of the heavy $\Lambda_{b}$ baryon induced by the flavor changing neutral currents. The rare $\Lambda_{b} \rightarrow \Lambda \ell^{+} \ell^{-}$ decays induced by the $b \rightarrow s$ transition were observed by the CDF [1] and LHCb collaborations [2]. Later the detailed analyses of the differential branching ratio and various symmetries of the $\Lambda_{b} \rightarrow \Lambda \ell^{+} \ell^{-}$decay have been performed performed at $\mathrm{LHCb}$ [3]. The LHCb collaboration firstly observed the rare $\Lambda_{b} \rightarrow p \pi^{-} \mu^{+} \mu^{-}$decay induced by the $b \rightarrow d$ transition [4]. This observation motivated the theoretical study of the $\Lambda_{b} \rightarrow N \ell^{+} \ell^{-}$decay, induced also by the $b \rightarrow d$ transition. This decay was studied within the framework of the light cone QCD sum rules method (LCSR) in [5]. The light cone QCD sum rules method (LCSR) [6,7] is hybrid of the traditional SVZ sum rules [8] and the methods used in hard exclusive processes. The other interesting decays induced by the $b \rightarrow d$ transition are the $\Lambda_{b} \rightarrow$ nucleon resonance decays. The analysis of these decays can provide complementary information about the properties of the nucleon resonances in principle which could experimentally be stud-

\footnotetext{
a e-mail: taliev@metu.edu.tr

b e-mail: tbarakat@ksu.edu.sa

ce-mail: savci@metu.edu.tr
}

ied at LHCb. It should be noted here that the comprehensive study of the nucleon resonance constitutes one of the main research directions of the research program that is planned for future study at Jefferson Laboratory [9]. The properties of the nucleon resonance $N^{*}$ in the $\Lambda_{b(c)} \rightarrow N^{*} \ell v$ decay is investigated in framework of the LCSR in [10]. The present work is devoted to the study of the rare $\Lambda_{b} \rightarrow N^{*} \ell^{+} \ell^{-}$ decay in the framework of the LCSR method.

The paper is organized as follows: In Sect. 2 the LCSR for the relevant form factors appearing in the $\Lambda_{b}\left(\Lambda_{b}^{*}\right) \rightarrow$ $N^{*}$ transitions are obtained. In Sect. 3 present the numerical analysis of the sum rules for the form factors. Using then the obtained results for the form factors we estimate the decay widths of the $\Lambda_{b}\left(\Lambda_{b}^{*}\right) \rightarrow N^{*} \ell^{+} \ell^{-}$decays. This section ends with a conclusion.

\section{Form factors of the $\Lambda_{b}\left(\Lambda_{b}^{*}\right) \rightarrow N^{*} \ell^{+} \ell^{-}$decay in LCSR}

In the present section we derive the LCSR for the transition form factors of the $\Lambda_{b}\left(\Lambda_{b}^{*}\right) \rightarrow N^{*} \ell^{+} \ell^{-}$decay. Before giving the details of the calculations few words about the notation should be mentioned. In all further discussions the negative parity states of the $\Lambda_{b}$ and $N$ baryons are denoted as $\Lambda_{b}^{*}$ and $N^{*}$, respectively. The $\Lambda_{b}\left(\Lambda_{b}^{*}\right) \rightarrow N^{*} \ell^{+} \ell^{-}$decay at the quark level is described by the $b \rightarrow d$ transition. At the hadronic level $\Lambda_{b}\left(\Lambda_{b}^{*}\right) \rightarrow N^{*} \ell^{+} \ell^{-}$decay is obtained by sandwiching the transition current between the $\Lambda_{b}\left(\Lambda_{b}^{*}\right)$ and $N^{*}$ states. The corresponding form factors of the vector, axial vector and tensor currents are defined as,

$$
\begin{aligned}
& \left\langle\Lambda_{b}(p-q)\left|\bar{b} \gamma_{\mu} d\right| N^{*}(p)\right\rangle \\
& =\bar{u}_{\Lambda_{b}}(p-q)\left[f_{1}\left(q^{2}\right) \gamma_{\mu}+i \frac{f_{2}\left(q^{2}\right)}{m_{\Lambda_{b}}} \sigma_{\mu \nu} q^{\nu}\right. \\
& \left.\quad+\frac{f_{3}\left(q^{2}\right)}{m_{\Lambda_{b}}} q^{\mu}\right] \gamma_{5} u_{N^{*}}(p),
\end{aligned}
$$




$$
\begin{aligned}
& \left\langle\Lambda_{b}(p-q)\left|\bar{b} \gamma_{\mu} \gamma_{5} d\right| N^{*}(p)\right\rangle \\
& =\bar{u}_{\Lambda_{b}}(p-q)\left[g_{1}\left(q^{2}\right) \gamma_{\mu} \gamma_{5}+i \frac{g_{2}\left(q^{2}\right)}{m_{\Lambda_{b}}} \sigma_{\mu \nu} q^{\nu} \gamma_{5}\right. \\
& \left.\quad+\frac{g_{3}\left(q^{2}\right)}{m_{\Lambda_{b}}} q^{\mu} \gamma_{5}\right] \gamma_{5} u_{N^{*}}(p), \\
& \left\langle\Lambda_{b}(p-q)\left|\bar{b} i \sigma_{\mu \nu} q^{\nu}\left(1+\gamma_{5}\right) d\right| N^{*}(p)\right\rangle \\
& =\bar{u}_{\Lambda_{b}}(p-q)\left[\frac{f_{1}^{T}\left(q^{2}\right)}{m_{\Lambda_{b}}}\left(\gamma_{\mu} q^{2}-q_{\mu} \not\right)+i f_{2}^{T}\left(q^{2}\right) \sigma_{\mu \nu} q^{\nu}\right. \\
& \left.\quad+\frac{g_{1}^{T}\left(q^{2}\right)}{m_{\Lambda_{b}}}\left(\gamma_{\mu} q^{2}-q_{\mu} \not q\right) \gamma_{5}+i g_{2}^{T}\left(q^{2}\right) \sigma_{\mu \nu} q^{v} \gamma_{5}\right] \gamma_{5} u_{N^{*}}(p) .
\end{aligned}
$$

The form factors responsible for $\Lambda_{b}^{*} \rightarrow N^{*}$ transition can be obtained from Eqs. (1)-(3) with the help of the following replacements: $f_{i} \rightarrow \widetilde{f}_{i}, g_{i} \rightarrow \widetilde{g}_{i}, f_{i}^{T} \rightarrow \widetilde{f}_{i}^{T}, g_{i}^{T} \rightarrow \widetilde{g}_{i}^{T}$, $m_{\Lambda_{b}} \rightarrow m_{\Lambda_{b}^{*}}$, and $\bar{u}_{\Lambda}(p-q) \rightarrow \bar{u}_{\Lambda^{*}}(p-q) \gamma_{5}$.

In order to derive the LCSR for these form factors we introduce the correlation function

$\Pi_{\mu}^{j}(p, q)=i \int d^{4} x e^{i q x}\left\langle 0\left|T\left\{\eta_{\Lambda_{b}}(0) J_{\mu}^{j}(x)\right\}\right| N^{*}(p)\right\rangle$,

where $\eta_{\Lambda_{b}}$ is the interpolating current of the $\Lambda_{b}\left(\Lambda_{b}^{*}\right)$ baryon, $J_{\mu}^{j}(x)$ is the heavy-light transition current which is set to,

$J_{\mu}^{j}= \begin{cases}\bar{b} \gamma_{\mu}\left(1-\gamma_{5}\right) d, & j=I \\ \bar{b} i \sigma_{\alpha \nu} q^{\nu}\left(1+\gamma_{5}\right) d, & j=I I .\end{cases}$

In the calculations we use the following most general form of the interpolating current for the $\Lambda_{b}\left(\Lambda_{b}^{*}\right)$ baryon,

$$
\begin{aligned}
\eta_{\Lambda_{b}}= & \frac{1}{\sqrt{6}} \epsilon_{a b c}\left\{2 \left(\left[u^{a T}(x) C d^{b}(x)\right] \gamma_{5} b^{c}(x)\right.\right. \\
& \left.+\beta\left[u^{a T}(x) C \gamma_{5} d^{b}(x)\right] b^{c}(x)\right) \\
& +\left[u^{a T}(x) C b^{b}(x)\right] \gamma_{5} d^{c}(x) \\
& +\beta\left[u^{a T}(x) C \gamma_{5} b^{b}(x)\right] d^{c}(x) \\
& +\left[b^{a T}(x) C d^{b}(x)\right] \gamma_{5} u^{c}(x) \\
& \left.+\beta\left[b^{a T}(x) C \gamma_{5} d^{b}(x)\right] u^{c}(x)\right\}
\end{aligned}
$$

where $a, b$ and $c$ are the color indices, $C$ is the charge conjugation operator, and $\beta$ is an arbitrary parameter with $\beta=-1$ corresponding to the Ioffe current.

The usual procedure in deriving the LCSR is to calculate the correlation function given in Eq. (4) in two different domains. On one side, insert a complete set of states with the quantum numbers of $\Lambda_{b}$ between the two currents, and isolate the ground state contribution. On the other side use the operator product expansion (OPE) around the light cone where $(p=q)^{2}, q^{2} \ll 0$. These two representations of the correlation function are then matched using the dispersion relations and quark-gluon duality ansatz. Finally, applying the Borel transformation in order to kill the the possible subtraction terms which could appear in the dispersion relations, and to suppress the contributions from higher states.

It should be remarked here that the interpolating current $\eta_{\Lambda_{b}}$ has nonzero overlap not only with the $J^{P}=\frac{1}{2}^{+}$state but also with the $J^{P}=\frac{1}{2}^{-}$state. It is shown in [11] that the mass difference between between the $J^{P}=\frac{1}{2}^{+}$and $J^{P}=\frac{1}{2}^{-}$states is about (200-300) MeV. For this reason the contribution of the negative parity $\Lambda_{b}$ baryon should properly be taken into account.

After having mentioned these cautionary remarks we proceed to calculate the physical part of the correlation function. Saturating Eq. (4) with the ground and first excited $\Lambda_{b}$ baryon we get,

$$
\begin{aligned}
& \Pi_{\mu}^{j}(p, q) \\
& =\sum_{i} \frac{\left\langle 0\left|\eta_{\Lambda_{b}}(0)\right| \Lambda_{b}^{i}(p-q, s)\right\rangle\left\langle\Lambda_{b}^{i}(p-q, s)\left|\bar{b} \Gamma_{\mu}^{j} d\right| N^{*}(p)\right\rangle}{m_{i}^{2}-(p-q)^{2}},
\end{aligned}
$$

where

$\Gamma_{\mu}^{j}= \begin{cases}\gamma_{\mu}\left(1-\gamma_{5}\right), & j=I, \\ i \sigma_{\mu \nu} q^{v}\left(1+\gamma_{5}\right), & j=I I,\end{cases}$

and summation is performed over the ground and first excited states of the $\Lambda_{b}$ baryon. The decay constants of the positive and negative parity $\Lambda_{b}$ baryons are determined as,

$$
\begin{aligned}
\left\langle 0\left|\eta_{\Lambda_{b}}\right| \Lambda_{b}(p-q)\right\rangle & =\lambda_{\Lambda_{b}} u_{\Lambda_{b}}(p-q), \\
\left\langle 0\left|\eta_{\Lambda_{b}^{*}}\right| \Lambda_{b}^{*}(p-q)\right\rangle & =\lambda_{\Lambda_{b}^{*}} \gamma_{5} u_{\Lambda_{b}^{*}}(p-q) .
\end{aligned}
$$

Using the equation of motion

$\left(\not p-m_{N^{*}}\right) u_{N^{*}}(p)=0$,

and Eqs. (1)-(3) and (7), for the phenomenological part of the correlation function we get,

$$
\begin{aligned}
\Pi_{\mu}^{I}(p, q)= & \frac{\lambda_{\Lambda_{b}}}{m_{\Lambda_{b}}^{2}-(p-q)^{2}}\left\{f _ { 1 } ( q ^ { 2 } ) \left[\left(m_{\Lambda_{b}}+m_{N^{*}}\right) \gamma_{\mu}\right.\right. \\
& \left.+\gamma_{\mu} \not q+2\left(p_{\mu}-q_{\mu}\right) I\right] \gamma_{5} \\
& -\frac{f_{2}\left(q^{2}\right)}{m_{\Lambda_{b}}}\left[( m _ { \Lambda _ { b } } - m _ { N ^ { * } } ) \left[\left(m_{\Lambda_{b}}+m_{N^{*}}\right) \gamma_{\mu}\right.\right. \\
& \left.+\gamma_{\mu} \not 1\right] \gamma_{5}+2 \not q \gamma_{5} p_{\mu} \\
& \left.+\left[\left(m_{\Lambda_{b}}-m_{N^{*}}\right)+\not\right] \gamma_{5} q_{\mu}\right] \\
& +\frac{f_{3}\left(q^{2}\right)}{m_{\Lambda_{b}}}\left[\left(m_{\Lambda_{b}}-m_{N^{*}}\right)-\not q\right] \gamma_{5} q_{\mu}
\end{aligned}
$$




$$
\begin{aligned}
& -g_{1}\left(q^{2}\right)\left[\left(m_{\Lambda_{b}}-m_{N^{*}}\right) \gamma_{\mu}+\gamma_{\mu} \not 1\right. \\
& \left.+2\left(p_{\mu}-q_{\mu}\right) I\right] \\
& +\frac{g_{2}\left(q^{2}\right)}{m_{\Lambda_{b}}}\left[\left(2 p_{\mu}-q_{\mu}\right) \not 1\right. \\
& +\left(m_{\Lambda_{b}}+m_{N^{*}}\right)\left[\left(m_{\Lambda_{b}}-m_{N^{*}}\right) \gamma_{\mu}\right. \\
& \left.\left.+\gamma_{\mu} \not 1-q_{\mu} I\right]\right] \\
& \left.+\frac{g_{3}\left(q^{2}\right)}{m_{\Lambda_{b}}}\left[\not q-\left(m_{\Lambda_{b}}+m_{N^{*}}\right)\right] q_{\mu} I\right\} \\
& +\frac{\lambda_{\Lambda_{b}^{*}}}{m_{\Lambda_{b}^{*}}^{2}-(p-q)^{2}}\left\{\widetilde { f _ { 1 } } ( q ^ { 2 } ) \left[\left(m_{\Lambda_{b}^{*}}-m_{N^{*}}\right) \gamma_{\mu}\right.\right. \\
& \left.-\gamma_{\mu} \not q-2\left(p_{\mu}-q_{\mu}\right) I\right] \gamma_{5} \\
& +\frac{\tilde{f}_{2}\left(q^{2}\right)}{m_{\Lambda_{b}^{*}}}\left[( m _ { \Lambda _ { b } ^ { * } } + m _ { N ^ { * } } ) \left[\left(m_{\Lambda_{b}^{*}}-m_{N^{*}}\right) \gamma_{\mu}\right.\right. \\
& \left.-\gamma_{\mu} \not\right] \gamma_{5}+2 \not p p_{\mu} \\
& \left.+\left[\left(m_{\Lambda_{b}^{*}}+m_{N^{*}}\right)-\not\right] \gamma_{5} q_{\mu}\right] \\
& +\frac{\widetilde{f_{3}}\left(q^{2}\right)}{m_{\Lambda_{b}^{*}}}\left[\left(m_{\Lambda_{b}^{*}}+m_{N^{*}}\right) I+\not 1\right] q_{\mu} \\
& -\widetilde{g}_{1}\left(q^{2}\right)\left[\left(m_{\Lambda_{b}^{*}}+m_{N^{*}}\right) \gamma_{\mu}\right. \\
& \left.-\gamma_{\mu} \not 1-2\left(p_{\mu}-q_{\mu}\right) I\right] \\
& -\frac{\widetilde{g}_{2}\left(q^{2}\right)}{m_{\Lambda_{b}^{*}}}\left[\left(2 p_{\mu}-q_{\mu}\right) \not 1\right. \\
& +\left(m_{\Lambda_{b}^{*}}-m_{N^{*}}\right)\left[\left(m_{\Lambda_{b}^{*}}+m_{N^{*}}\right) \gamma_{\mu}\right. \\
& \left.\left.-\gamma_{\mu} \not 1+q_{\mu} I\right]\right] \\
& -\frac{\widetilde{g}_{3}\left(q^{2}\right)}{m_{\Lambda_{b}^{*}}}\left[\not q+\left(m_{\Lambda_{b}^{*}}-m_{N^{*}}\right) I\right] q_{\mu},
\end{aligned}
$$$$
\Pi_{\mu}^{I I}(p, q)=\frac{\lambda_{\Lambda_{b}}}{m_{\Lambda_{b}}^{2}-(p-q)^{2}}\left\{\frac { f _ { 1 } ^ { T } ( q ^ { 2 } ) } { m _ { \Lambda _ { b } } } \left[\left[\left(m_{\Lambda_{b}}+m_{N^{*}}\right) \gamma_{\mu}\right.\right.\right.
$$$$
\left.+\gamma_{\mu} \not 1+2 I p_{\mu}\right] \gamma_{5} q^{2}
$$$$
+\left[\left(m_{\Lambda_{b}}^{2}-m_{N^{*}}^{2}-2 q^{2}\right)\right.
$$$$
\left.\left.\left.-\left(m_{\Lambda_{b}}+m_{N^{*}}\right) \not\right]\right]\right] \gamma_{5} q_{\mu}
$$$$
-f_{2}^{T}\left(q^{2}\right)\left[( m _ { \Lambda _ { b } } - m _ { N ^ { * } } ) \left[\left(m_{\Lambda_{b}}+m_{N^{*}}\right) \gamma_{\mu}\right.\right.
$$$$
\left.+\gamma_{\mu} \not\right] \gamma_{5}+2 \not \gamma_{5} p_{\mu}
$$$$
\left.-\left[\left(m_{\Lambda_{b}}-m_{N^{*}}\right)+\not q\right] \gamma_{5} q_{\mu}\right]
$$$$
+\frac{g_{1}^{T}\left(q^{2}\right)}{m_{\Lambda_{b}}}\left[\left[\left(m_{\Lambda_{b}}-m_{N^{*}}\right) \gamma_{\mu}\right.\right.
$$$$
\left.+\gamma_{\mu} \not q+2 I p_{\mu}\right] q^{2}-\left[\left(m_{\Lambda_{b}}-m_{N^{*}}\right) \not 1\right.
$$$$
\left.\left.-\left(m_{\Lambda_{b}}^{2}-m_{N^{*}}^{2}-2 q^{2}\right)\right] q_{\mu}\right]
$$

$$
\begin{aligned}
& -g_{2}^{T}\left(q^{2}\right)\left[\left(2 p_{\mu}-q_{\mu}\right) \not q+\left(m_{\Lambda_{b}}+m_{N^{*}}\right)\right. \\
& \left.\left.\left[\left(m_{\Lambda_{b}}-m_{N^{*}}\right) \gamma_{\mu}+\gamma_{\mu} \not q-q_{\mu}\right]\right]\right\} \\
& +\frac{\lambda_{\Lambda_{b}^{*}}}{m_{\Lambda_{b}^{*}}^{2}-(p-q)^{2}} \\
& \left\{\frac { \tilde { f } _ { 1 } ^ { T } ( q ^ { 2 } ) } { m _ { \Lambda _ { b } ^ { * } } } \left[\left[\left(m_{\Lambda_{b}^{*}}-m_{N^{*}}\right) \gamma_{\mu}\right.\right.\right. \\
& \left.-\gamma_{\mu} \not 1-2 I p_{\mu}\right] q^{2} \gamma_{5} \\
& -\left[\left(m_{\Lambda_{b}^{*}}^{2}-m_{N^{*}}^{2}-2 q^{2}\right)\right. \\
& \left.\left.+\left(m_{\Lambda_{b}^{*}}-m_{N^{*}}\right) \not 1\right] \gamma_{5} q_{\mu}\right] \\
& +\widetilde{f}_{2}^{T}\left(q^{2}\right)\left[( m _ { \Lambda _ { b } ^ { * } } + m _ { \Lambda _ { b } } ^ { * } ) \left[\left(m_{\Lambda_{b}^{*}}-m_{\Lambda_{b}}^{*}\right) \gamma_{\mu}\right.\right. \\
& \left.-\gamma_{\mu} \not\right] \gamma_{5}+2 \not 1 \gamma_{5} p_{\mu} \\
& +\left[\left(m_{\Lambda_{b}^{*}}+m_{\Lambda_{b}}^{*}\right)-\not q \gamma_{5} q_{\mu}\right] \\
& +\frac{\widetilde{g}_{1}^{T}\left(q^{2}\right)}{m_{\Lambda_{b}^{*}}}\left[\left[\left(m_{\Lambda_{b}^{*}}+m_{N^{*}}\right) \gamma_{\mu}-\gamma_{\mu} \not\right.\right. \\
& \left.-2 I p_{\mu}\right] q^{2}-\left[\left(m_{\Lambda_{b}^{*}}+m_{N^{*}}\right) \not 1\right. \\
& \left.\left.+\left(m_{\Lambda_{b}^{*}}^{2}-m_{N^{*}}^{2}-2 q^{2}\right)\right] q_{\mu}\right] \\
& +\widetilde{g}_{2}^{T}\left(q^{2}\right)\left[\left(2 p_{\mu}-q_{\mu}\right) \not 1\right. \\
& +\left(m_{\Lambda_{b}^{*}}-m_{N^{*}}\right)\left[\left(m_{\Lambda_{b}^{*}}+m_{N^{*}}\right) \gamma_{\mu}\right. \\
& \left.\left.\left.-\gamma_{\mu} \not 1+I q_{\mu}\right]\right]\right\} \text {. }
\end{aligned}
$$

Now we turn our attention to the calculation of the correlation function from the QCD side. At deep Eucledian domain $(p-q)^{2}, q^{2} \ll 0$ the product of the two currents can be expanded around the light-cone $x^{2} \simeq 0$. After contracting the heavy quark fields which give the heavy quark propagator, the matrix element

$\epsilon^{a b c}\left\langle 0\left|u_{\alpha}^{a}(0) d_{\beta}^{b}(x) d_{\lambda}^{c}(0)\right| N^{*}(p)\right\rangle$,

of the three quarks between the vacuum and the $N^{*}$ state is revealed. Decomposition of this matrix element in terms of the distribution amplitudes (DAs) with increasing twist is given in [12] (see Appendix A).

After contracting the heavy b-quark fields, the correlation takes the form,

$$
\begin{aligned}
\Pi_{\mu}^{j}= & \frac{i}{\sqrt{6}} \epsilon^{a b c} \int d^{4} x e^{i q x} \\
& \left\{\left[2(C)_{\alpha \lambda}\left(\gamma_{5}\right)_{\rho \xi}+(C)_{\alpha \xi}\left(\gamma_{5}\right)_{\rho \lambda}+(C)_{\xi \lambda}\left(\gamma_{5}\right)_{\alpha \rho}\right]\right. \\
& +\beta\left[2\left(C \gamma_{5}\right)_{\alpha \lambda}(I)_{\rho \xi}+\left(C \gamma_{5}\right)_{\alpha \xi}(I)_{\rho \lambda}\right.
\end{aligned}
$$




$$
\begin{aligned}
&+\left.\left.\left(C \gamma_{5}\right)_{\rho \lambda}(I)_{\alpha \rho}\right]\right\} \times\left(\Gamma_{\mu}^{j}\right)_{\sigma \beta}\left[S_{b}(-x)\right]_{\xi \sigma} \\
&\left\langle 0\left|u_{\alpha}^{a}(0) d_{\beta}^{b}(x) d_{\lambda}^{c}(0)\right| N^{*}(p)\right\rangle \\
& S_{b}(x)= \frac{m_{b}^{2}}{4 \pi^{2}} \frac{K_{1}\left(m_{b} \sqrt{-x^{2}}\right)}{\sqrt{-x^{2}}}-i \frac{m_{b}^{2} \not x}{4 \pi^{2} x^{2}} K_{2}\left(m_{b} \sqrt{-x^{2}}\right) \\
&-i g_{s} \int \frac{d^{4} k}{(2 \pi)^{4}} e^{-i k x} \int_{0}^{1} d v \\
& {\left[\frac{\not k+m_{b}}{\left(m_{b}^{2}-k^{2}\right)^{2}} G^{\alpha \beta}(v x) \sigma_{\alpha \beta}+\frac{1}{m_{b}^{2}-k^{2}} v x_{\alpha} G^{\alpha \beta} \gamma_{\beta}\right], }
\end{aligned}
$$

where $K_{i}$ are the modified Bessel functions of the second kind, and $G_{\alpha \beta}$ is the gluon field strength tensor.

Using the expression of the heavy quark propagator and definition of the matrix

$\epsilon^{a b c}\left\langle 0\left|u_{\alpha}^{a}(0) d_{\beta}^{b}(x) d_{\lambda}^{c}(0)\right| N^{*}(p)\right\rangle$,

in terms of the DAs of the $N^{*}$ baryon, we can calculate the correlation function from the QCD side. Note that, using the equation of motion $\left(\not p-m_{N^{*}}\right) u_{N^{*}}(p)=0$, the correlation function can be decomposed into six independent functions as follows,

$$
\begin{aligned}
\Pi_{\mu}^{j}(p, q)= & {\left[\Pi_{1}^{j} p_{\mu}+\Pi_{2}^{j} p_{\mu} \not 1+\Pi_{3}^{j} \gamma_{\mu}\right.} \\
& \left.+\Pi_{4}^{j} \gamma_{\mu} \not 1+\Pi_{5}^{j} q_{\mu}+\Pi_{6}^{j} q_{\mu} \not 1\right] \gamma_{5} u_{N^{*}} .
\end{aligned}
$$

After performing Borel transformation, these invariant functions in the correlation function can in general be written as,

$$
\Pi_{i}^{j}\left[(p-q)^{2}, q^{2}\right]=\sum_{n=1,2,3} \int_{0}^{1} \frac{\mathcal{D}_{i n}^{j}\left(x, q^{2}\right)}{\Delta^{n}},
$$

where

$$
\Delta=m_{b}^{2}-(1-x) q^{2}+x(1-x) m_{N^{*}}^{2}-x(p-q)^{2} .
$$

We can write (13) as a dispersion integral in $(p-q)^{2}$ as follows,

$$
\begin{aligned}
& \Pi_{i}^{j}\left[(p-q)^{2}, q^{2}\right] \\
& \quad=\frac{1}{\pi} \int_{m_{b}^{2}}^{\infty} \frac{d s}{s-(p-q)^{2}} \operatorname{Im} \Pi_{i}^{j}\left[(p-q)^{2}, q^{2}\right] .
\end{aligned}
$$

Making the replacement $s(x)=m_{b}^{2}-(1-x) q^{2}+x(1-$ $x) m_{N^{*}}^{2}-x(p-q)^{2}$, the denominator of Eq. (13) takes the form,

$$
\Delta=x\left[s(x)-(p-q)^{2}\right] \text {. }
$$

Using the quark-hadron ansatz the contribution of the hadronic states can be represented as,

$$
\begin{aligned}
& \int_{s_{0}}^{\infty} \frac{d s}{s-(p-q)^{2}} \rho_{i}\left(q^{2}\right) \\
& \quad \simeq \frac{1}{\pi} \int_{s_{0}}^{\infty} \frac{d s}{s-(p-q)^{2}} \operatorname{Im} \Pi_{i}^{j}\left(q^{2}\right)
\end{aligned}
$$

where $s_{0}$ is the continuum contribution. Finally Borel transformation can be performed on the hadronic and physical sides with the help of the replacement

$\frac{1}{s-(p-q)^{2}} \rightarrow e^{-s / M^{2}}$.

In implementing the Borel transformation and the continuum subtraction we use the following relations,

$$
\begin{aligned}
& \int d x \frac{\mathcal{D}_{i 1}^{j}(x)}{\Delta} \rightarrow \int_{x_{0}}^{1} \frac{d x}{x} \mathcal{D}_{i 1}^{j}(x) e^{-s(x) / M^{2}} \\
& \int d x \frac{\mathcal{D}_{i 2}^{j}(x)}{\Delta^{2}} \rightarrow \frac{1}{M^{2}} \int_{x_{0}}^{1} \frac{d x}{x^{2}} \mathcal{D}_{i 2}^{j}(x) e^{-s(x) / M^{2}} \\
& +\frac{\mathcal{D}_{i 2}^{j}\left(x_{0}\right) e^{-s_{0} / M^{2}}}{m_{b}^{2}+x_{0}^{2} m_{N^{*}}^{2}-q^{2}} \\
& \int d x \frac{\mathcal{D}_{i 3}^{j}(x)}{\Delta^{3}} \rightarrow \frac{1}{2 M^{4}} \int_{x_{0}}^{1} \frac{d x}{x^{3}} \mathcal{D}_{i 3}^{j}(x) e^{-s(x) / M^{2}} \\
& +\frac{1}{2 M^{2}} \frac{\mathcal{D}_{i 3}^{j}\left(x_{0}\right) e^{-s_{0} / M^{2}}}{x_{0}\left(m_{b}^{2}+x_{0}^{2} m_{N^{*}}^{2}-q^{2}\right)} \\
& -\left.\frac{1}{2} \frac{x_{0}^{2} e^{-s_{0} / M^{2}}}{m_{b}^{2}+x_{0}^{2} m_{N^{*}}^{2}-q^{2}} \frac{d}{d x}\left(\frac{\mathcal{D}_{i 3}^{j}(x)}{x\left(m_{b}^{2}+x^{2} m_{N^{*}}^{2}-q^{2}\right)}\right)\right|_{x=x_{0}}
\end{aligned}
$$

where $x_{0}$ is the solution of the equation

$s_{0}=\frac{m_{b}^{2}-\bar{x} q^{2}+x \bar{x} m_{N^{*}}^{2}}{x}$.

Equating the coefficients of the structures $p_{\mu} \gamma_{5}, p_{\mu} q \gamma_{5}$, $\gamma_{\mu} \gamma_{5}, \gamma_{\mu} q_{\gamma_{5}}, q_{\mu} \gamma_{5}$, and $q_{\mu} q \gamma_{5}$ we get the following sum rules for the invariant functions of the transition current $\left(\bar{b} \gamma_{\mu} d\right)$,

$$
\begin{aligned}
- & 2 \lambda_{\Lambda_{b}} g_{1}\left(q^{2}\right) e^{-m_{\Lambda_{b}}^{2} / M^{2}}+2 \lambda_{\Lambda_{b}^{*}} \widetilde{g}_{1}\left(q^{2}\right) e^{-m_{\Lambda_{b}^{*}}^{2} / M^{2}} \\
= & \Pi_{1}^{I}(p, q) \\
& 2 \lambda_{\Lambda_{b}} \frac{g_{2}\left(q^{2}\right)}{m_{\Lambda_{b}}} e^{-m_{\Lambda_{b}}^{2} / M^{2}}-2 \lambda_{\Lambda_{b}^{*}} \frac{\widetilde{g}_{2}\left(q^{2}\right)}{m_{\Lambda_{b}^{*}}} e^{-m_{\Lambda_{b}^{*}}^{2} / M^{2}} \\
= & \Pi_{2}^{I}(p, q) \\
& -\lambda_{\Lambda_{b}} e^{-m_{\Lambda_{b}}^{2} / M^{2}}\left\{( m _ { \Lambda _ { b } } - m _ { N ^ { * } } ) \left[g_{1}\left(q^{2}\right)\right.\right. \\
& \left.\left.-\frac{g_{2}\left(q^{2}\right)}{m_{\Lambda_{b}}}\left(m_{\Lambda_{b}}+m_{N^{*}}\right)\right]\right\} \\
- & \lambda_{\Lambda_{b}^{*}} e^{-m_{\Lambda_{b}^{*}}^{2} / M^{2}}\left\{( m _ { \Lambda _ { b } ^ { * } } + m _ { N ^ { * } } ) \left[\widetilde{g}_{1}\left(q^{2}\right)\right.\right.
\end{aligned}
$$




$$
\begin{aligned}
& \left.\left.+\frac{\widetilde{g}_{2}\left(q^{2}\right)}{m_{\Lambda_{b}^{*}}}\left(m_{\Lambda_{b}^{*}}-m_{N^{*}}\right)\right]\right\}=\Pi_{3}^{I}(p, q) \\
& -\lambda_{\Lambda_{b}} e^{-m_{\Lambda_{b}}^{2} / M^{2}}\left[g_{1}\left(q^{2}\right)-\frac{g_{2}\left(q^{2}\right)}{m_{\Lambda_{b}}}\left(m_{\Lambda_{b}}+m_{N^{*}}\right)\right] \\
& +\lambda_{\Lambda_{b}^{*}} e^{-m_{\Lambda_{b}^{*}}^{2} / M^{2}}\left[\widetilde{g}_{1}\left(q^{2}\right)+\frac{\tilde{g}_{2}\left(q^{2}\right)}{m_{\Lambda_{b}^{*}}}\left(m_{\Lambda_{b}^{*}}-m_{N^{*}}\right)\right] \\
& =\Pi_{4}^{I}(p, q) \\
& \lambda_{\Lambda_{b}} e^{-m_{\Lambda_{b}}^{2} / M^{2}}\left[2 g_{1}\left(q^{2}\right)\right. \\
& \left.-\frac{g_{2}\left(q^{2}\right)+g_{3}\left(q^{2}\right)}{m_{\Lambda_{b}}}\left(m_{\Lambda_{b}^{*}}+m_{N^{*}}\right)\right]
\end{aligned}
$$

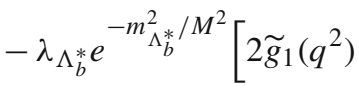

$$
\begin{aligned}
& \left.+\frac{\widetilde{g}_{2}\left(q^{2}\right)+\widetilde{f}_{3}\left(q^{2}\right)}{m_{\Lambda_{b}^{*}}}\left(m_{\Lambda_{b}^{*}}-m_{N^{*}}\right)\right]=\Pi_{5}^{I}(p, q) \\
& -\frac{\lambda_{\Lambda_{b}}}{m_{\Lambda_{b}}} e^{-m_{\Lambda_{b}}^{2} / M^{2}}\left[g_{2}\left(q^{2}\right)-g_{3}\left(q^{2}\right)\right] \\
& +\frac{\lambda_{\Lambda_{b}^{*}}}{m_{\Lambda_{b}^{*}}} e^{-m_{\Lambda_{b}^{*}}^{2} / M^{2}}\left[\tilde{g}_{2}\left(q^{2}\right)-\widetilde{g}_{3}\left(q^{2}\right)\right]=\Pi_{6}^{I}(p, q) \text {. }
\end{aligned}
$$

The results for the form factors induced by the $\bar{b} \gamma_{\mu} \gamma_{5} d$ current can be obtained from Eq. (18) by making the following replacements: $g_{i} \rightarrow-f_{i}, \widetilde{g}_{i} \rightarrow-\widetilde{f}_{i}, m_{N^{*}} \rightarrow-m_{N^{*}}$, and $\Pi_{i}^{I} \rightarrow \Pi_{i}^{I \prime}$.

The sum rules of the $\left(\bar{b} i \sigma_{\mu \nu} q^{v} d\right)$ transition current can be obtained in similar manner, which are given below,

$$
\begin{aligned}
- & 2 \lambda_{\Lambda_{b}} g_{2}^{T}\left(q^{2}\right) e^{-m_{\Lambda_{b}}^{2} / M^{2}}+2 \lambda_{\Lambda_{b}^{*}} \widetilde{g}_{2}^{T}\left(q^{2}\right) e^{-m_{\Lambda_{b}^{*}}^{2} / M^{2}} \\
= & \Pi_{1}^{I I}(p, q) \\
& -\lambda_{\Lambda_{b}} e^{-m_{\Lambda_{b}}^{2} / M^{2}}\left[\frac{g_{1}^{T}\left(q^{2}\right)}{m_{\Lambda_{b}}}\left(m_{\Lambda_{b}}-m_{N^{*}}\right)-g_{2}^{T}\left(q^{2}\right)\right] \\
& -\lambda_{\Lambda_{b}^{*}} e^{-m_{\Lambda_{b}^{*}}^{2} / M^{2}}\left[\frac{\widetilde{g}_{1}^{T}\left(q^{2}\right)}{m_{\Lambda_{b}^{*}}}\left(m_{\Lambda_{b}^{*}}+m_{N^{*}}\right)+\widetilde{g}_{2}^{T}\left(q^{2}\right)\right] \\
= & \Pi_{2}^{I I}(p, q) \\
& \lambda_{\Lambda_{b}} e^{-m_{\Lambda_{b}}^{2} / M^{2}}\left[\frac{g_{1}^{T}\left(q^{2}\right)}{m_{\Lambda_{b}}}\left(m_{\Lambda_{b}}^{2}-m_{N^{*}}^{2}-2 q^{2}\right)\right. \\
& \left.+g_{2}^{T}\left(q^{2}\right)\left(m_{\Lambda_{b}}+m_{N^{*}}\right)\right] \\
& -\lambda_{\Lambda_{b}^{*}} e^{-m_{\Lambda_{b}^{*} / M^{2}}^{2}}\left[\frac{\widetilde{g}_{1}^{T}\left(q^{2}\right)}{m_{\Lambda_{b}^{*}}}\left(m_{\Lambda_{b}^{*}}^{2}-m_{N^{*}}^{2}-2 q^{2}\right)\right. \\
& \left.-\widetilde{g}_{2}^{T}\left(q^{2}\right)\left(m_{\Lambda_{b}^{*}}-m_{N^{*}}\right)\right]=\Pi_{3}^{I I}(p, q) \\
& \lambda_{\Lambda_{b}} e^{-m_{\Lambda_{b} / M^{2}}^{2}\left(m_{\Lambda_{b}}-m_{N^{*}}\right)\left[\frac{g_{1}^{T}\left(q^{2}\right)}{m_{\Lambda_{b}}} q^{2}\right.} \\
& \left.-g_{2}^{T}\left(q^{2}\right)\left(m_{\Lambda_{b}}+m_{N^{*}}\right)\right]
\end{aligned}
$$

$$
\begin{aligned}
& +\lambda_{\Lambda_{b}^{*}} e^{\frac{-m_{\Lambda_{b}^{*}}^{2}}{M^{2}}}\left(m_{\Lambda_{b}^{*}}+m_{N^{*}}\right)\left[\frac{\widetilde{g}_{1}^{T}\left(q^{2}\right)}{m_{\Lambda_{b}^{*}}} q^{2}\right. \\
& \left.+\widetilde{g}_{2}^{T}\left(q^{2}\right)\left(m_{\Lambda_{b}^{*}}-m_{N^{*}}\right)\right]=\Pi_{4}^{I I}(p, q),
\end{aligned}
$$

where $\Pi_{1}^{I I}, \Pi_{2}^{I I}, \Pi_{3}^{I I}$, and $\Pi_{4}^{I I}$ are the invariant functions for the structures $p_{\mu} q \gamma_{5}, q_{\mu} q \gamma_{5}, q_{\mu} \gamma_{5}$, and $\gamma_{\mu} \gamma_{5}$, respectively. The sum rules for the $\left(\bar{b} i \sigma_{\mu \nu} q^{v} \gamma_{5} d\right)$ transition current can be obtained from Eq. (19) by making the replacements $g_{i}^{T} \rightarrow f_{i}^{T}, \widetilde{g}_{i}^{T} \rightarrow \tilde{f}_{i}^{T}, m_{N^{*}} \rightarrow-m_{N^{*}}$, and $\Pi_{i}^{I I} \rightarrow \Pi_{i}^{I I \prime}$. The explicit form of these invariant functions for the aforementioned structures are presented in Appendix-B.

Solving these equations we can eliminate the $\Lambda^{*}$ pole from the sum rules. As the result we obtain the desired sum rules responsible for the $\Lambda_{b} \rightarrow N^{*}$ transition. In the next section we present our numerical results on these form factors.

In full QCD for describing $\Lambda_{b} \rightarrow N^{*}$ transition we have 10 independent form factors. The number of independent form factors for $\Lambda_{b} \rightarrow N^{*}$ transitions reduces considerably in the heavy quark limit. Using the heavy quark symmetry and the soft collinear effective theory in [13-15] it is obtained that the matrix element for $\Lambda_{b} \rightarrow N^{*}$ transition can be in terms of only one universal function $\xi(p v)$.

$$
\left\langle N^{*}(p)|d \Gamma b| \Lambda_{b}\left(p^{\prime}\right)\right\rangle=\xi(p v) \bar{u}_{N^{*}}(p) \Gamma u_{\Lambda_{b}}(p)
$$

to the leading order. Using this relation and the definitions of the matrix elements in full QCD presented in Eqs. (1)-(3), we immediately get the following relations among the form factors:

$$
\begin{aligned}
f_{1}\left(q^{2}\right) & =g_{1}\left(q^{2}\right)=f_{2}^{T}\left(q^{2}\right)=g_{2}^{T}\left(q^{2}\right)=\xi(p v), \\
f_{2}\left(q^{2}\right) & =g_{2}\left(q^{2}\right)=f_{3}\left(q^{2}\right)=g_{3}\left(q^{2}\right) \\
& =f_{1}^{T}\left(q^{2}\right)=f_{2}^{T}\left(q^{2}\right)=0
\end{aligned}
$$

At the end of this section, we would like to make the following remark. For a complete description of the heavy-to-light baryon transition form factors, it is necessary to take into account non-factorizable contributions. The non-factorizable contribution is described by following non-local matrix element:

$h_{\mu}=i \int d^{4} x e^{i q x}\left\langle\Lambda_{b}\left(p^{\prime}\right)\left|j_{\mu}^{\mathrm{el}}(x) H_{\mathrm{eff}}(0)\right| N^{*}(p)\right\rangle$.

The $h_{\mu}$ generated by the including to the matrix element of the four-quark operators with $\Delta B=1$ effective weak Hamiltonian together with electromagnetic current for relevant quarks. The matrix element $h_{\mu}$ does not factorize into multiplication of leptonic current and form factors. The contributions can be described by "hard spectator scattering", "weak annihilation", "hard vertex corrections", "soft gluon radiations" (see Fig. 3. These figures are taken from 
Table 1 The parametrization of the form factors of the $\Lambda_{b} \rightarrow N^{*} \ell^{+} \ell^{-}$decay for LQSR-1

\begin{tabular}{lcrrr}
\hline & \multicolumn{1}{l}{$f_{i}(0)$} & \multicolumn{1}{c}{$a_{0}$} & \multicolumn{1}{c}{$a_{1}$} \\
\hline$f_{1}$ & $-0.297 \pm 0.080$ & $0.44 \pm 0.11$ & $-0.17 \pm 0.03$ & $4.16 \pm 0.40$ \\
$f_{2}$ & $-0.213 \pm 0.064$ & $-0.15 \pm 0.03$ & $-1.36 \pm 0.26$ & $5.22 \pm 0.90$ \\
$f_{3}$ & $-0.060 \pm 0.018$ & $0.74 \pm 0.10$ & $-7.24 \pm 1.20$ & $16.22 \pm 2.20$ \\
$g_{1}$ & $-0.028 \pm 0.084$ & $-0.35 \pm 0.06$ & $2.97 \pm 0.60$ & $-6.87 \pm 0.95$ \\
$g_{2}$ & $0.106 \pm 0.031$ & $0.31 \pm 0.05$ & $-0.93 \pm 0.16$ & $-0.37 \pm 0.04$ \\
$g_{3}$ & $-0.017 \pm 0.005$ & $-0.19 \pm 0.04$ & $1.66 \pm 0.31$ & $-3.98 \pm 0.70$ \\
$f_{1}^{T}$ & $-0.0030 \pm 0.0008$ & $4.5 \pm 0.9$ & $-43 \pm 8$ & $102 \pm 20$ \\
$f_{2}^{T}$ & $-0.190 \pm 0.057$ & $-3.3 \pm 0.6$ & $29 \pm 6$ & $-68 \pm 13$ \\
$g_{1}^{T}$ & $0.384 \pm 0.110$ & $-1.66 \pm 0.30$ & $19.5 \pm 3.5$ & $46 \pm 9$ \\
$g_{2}^{T}$ & $-0.190 \pm 0.056$ & $1.9 \pm 0.36$ & $-20 \pm 4$ & $47 \pm 9$ \\
\hline
\end{tabular}

Table 2 The same as Table 1, but for LQSR-2

\begin{tabular}{|c|c|c|c|c|}
\hline & $f_{i}(0)$ & $a_{0}$ & $a_{1}$ & $a_{2}$ \\
\hline$f_{1}$ & $-0.202 \pm 0.060$ & $-0.27 \pm 0.06$ & $-0.17 \pm 0.03$ & $2.37 \pm 0.33$ \\
\hline$f_{2}$ & $-0.0640 \pm 0.0018$ & $-0.060 \pm 0.012$ & $-2.87 \pm 0.50$ & $1.20 \pm 0.20$ \\
\hline$f_{3}$ & $0.0500 \pm 0.0015$ & $0.60 \pm 0.14$ & $-4.52 \pm 0.80$ & $9.07 \pm 1.50$ \\
\hline$g_{1}$ & $-0.144 \pm 0.043$ & $-0.38 \pm 0.06$ & $1.65 \pm 0.22$ & $-2.51 \pm 0.42$ \\
\hline$g_{2}$ & $0.062 \pm 0.002$ & $0.24 \pm 0.04$ & $-1.08 \pm 0.15$ & $-1.17 \pm 0.20$ \\
\hline$g_{3}$ & $-0.032 \pm 0.001$ & $-0.20 \pm 0.03$ & $1.47 \pm 0.23$ & $-3.11 \pm 0.50$ \\
\hline$f_{1}^{T}$ & $0.0210 \pm 0.0063$ & $0.27 \pm 0.05$ & $-1.3 \pm 0.26$ & $0.55 \pm 0.10$ \\
\hline$f_{2}^{T}$ & $-0.176 \pm 0.052$ & $-0.57 \pm 0.11$ & $1.86 \pm 0.35$ & $0.04 \pm 0.008$ \\
\hline$g_{1}^{T}$ & $0.203 \pm 0.060$ & $0.56 \pm 0.10$ & $-1.71 \pm 0.34$ & $0.023 \pm 0.004$ \\
\hline$g_{2}^{T}$ & $-0.176 \pm 0.052$ & $-0.19 \pm 0.03$ & $0.078 \pm 0.015$ & $-0.030 \pm 0.006$ \\
\hline
\end{tabular}

[16]). For calculations of these contributions some nonperturbative approaches are needed (More details about the non-factorizable effects, see [15]).

In the present work, we calculate only factorizable contributions and we plan to present a detailed analysis of the non-factorizable contributions elsewhere in future.

\section{Numerical analysis}

In this section we present our numerical results on the form factors that describe the $\Lambda_{b} \rightarrow N^{*}$ transition. First let us specify the input parameters which are needed in performing the numerical calculations. The masses of the $\Lambda_{b}$ and $\Lambda_{b}^{*}$ baryons which we use in our calculations are $\Lambda_{b}=5.62 \mathrm{GeV}$ and $\Lambda_{b}^{*}=5.85 \mathrm{GeV}$, and the mass of the nucleon is $m_{N^{*}}=1.52 \mathrm{GeV}$ [17]. The residues $\lambda_{\Lambda_{b}}$ and $\lambda_{\Lambda_{b}^{*}}$ of the relevant baryons are taken from [5] having the values $\lambda_{\Lambda_{b}}=(6.5 \pm 1.5) \times 10^{-2} \mathrm{GeV}^{3}$ and $\lambda_{\Lambda_{b}^{*}}=(7.5 \pm 2.0) \times 10^{-2} \mathrm{GeV}^{3}$. The mass of the $b$ quark is assigned to its $\overline{M S}$ given as $m_{b}=(4.16 \pm 0.03) \mathrm{GeV}$ [17]. The values of the quark condensates of the light quarks are taken as, $\langle\bar{u} u\rangle(1 \mathrm{GeV})=\langle\bar{d} d\rangle(1 \mathrm{GeV})=-\left(246_{-19}^{+28} \mathrm{MeV}\right)^{3}$. As has already been noted the main nonperturbative param- eters are DAs of the $N^{*}$ baryon. The expressions of the $N^{*}$ DAs, as well as the coefficients $\phi_{i}^{( \pm, 0)}, \psi_{i}^{( \pm, 0)}$, and $\xi_{i}^{( \pm, 0)}$, appearing in the DAs are obtained in [12] (also in [1823]), and for completeness they are presented in Appendix A.

The sum rules for the transition form factors contain three auxiliary parameters: The Borel mass parameter $M^{2}$, the continuum threshold $s_{0}$ and the arbitrary parameter $\beta$. The Borel mass parameter and the continuum threshold $s_{0}$ are determined from the criteria that the sum rule dictates, i.e., the suppression of the contributions coming from the continuum states and the higher twist contributions should be satisfied. Our analysis shows that the working regions of $M^{2}$ and $s_{0}$ lie in the region $M^{2}=(10 \pm 5) \mathrm{GeV}^{2}, s_{0}=(40 \pm 1) \mathrm{GeV}^{2}$, when aforementioned conditions are fulfilled, and hence sum rules predictions are reliable. The final step of our analysis is is the determination of the working region of the parameter $\beta$. Our numerical study shows that when $-1 \leq \cos \theta \leq-0.5$, where $\tan \theta=\beta$ the results for the residues and masses are rather stable with respect to the variation of $\beta$, and we choose $\beta=-1$.

The LCSR predictions are reliable up to the range $q^{2} \leq$ $q_{\max }^{2}=\left(m_{\Lambda_{b}}-m_{N^{*}}\right)^{2}$. In order to calculate the decay width the LCSR predictions for the form factors need to be extrap- 
Fig. 1 The dependence of the differential branching ratio for the $\Lambda_{b} \rightarrow N^{*} \mu^{+} \mu^{-}$transition on $s$, at $s_{0}=40 \mathrm{GeV}^{2}$, and $M^{2}=25 \mathrm{GeV}^{2}$

Fig. 2 The same as in Fig. 1, but for the $\Lambda_{b} \rightarrow N^{*} \tau^{+} \tau^{-}$ transition
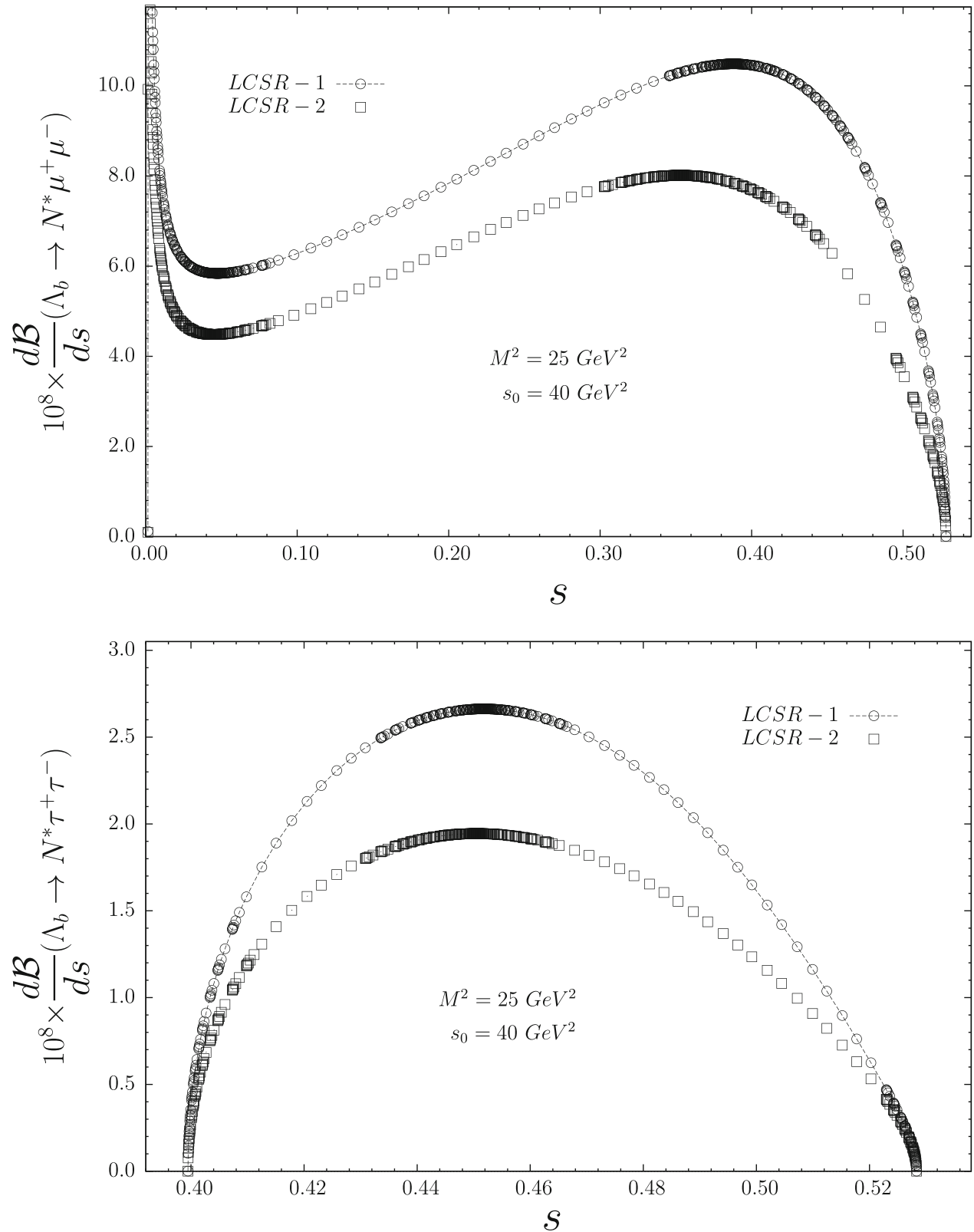

olated to the whole physical region. For this purpose we use the $\mathrm{z}$-series parametrization that is proposed in [24],

$z\left(q^{2}, t_{0}\right)=\frac{\sqrt{t_{+}-q^{2}}-\sqrt{t_{+}-t_{0}}}{\sqrt{t_{+}-q^{2}}+\sqrt{t_{+}-t_{0}}}$,

where $t_{0}=\left(m_{\Lambda_{b}}-m_{N^{*}}\right)^{2}, t_{+}=\left(m_{B}+m_{\pi}\right)^{2}$. The parametrization that best reproduces the form factors predicted by the LCSR in the region $q^{2} \leq 11 \mathrm{GeV}^{2}$, is given as

$$
\begin{aligned}
f\left(q^{2}\right)= & \frac{1}{1-q^{2} /\left(m_{\text {pole }}^{f}\right)^{2}} \\
& \left\{a_{0}^{f}+a_{1}^{f} z\left(q^{2}, t_{0}\right)+a_{2}^{f}\left[z\left(q^{2}, t_{0}\right)\right]^{2}\right\} .
\end{aligned}
$$

For the pole masses we use,

$$
m_{\text {pole }}=\left\{\begin{array}{l}
m_{B^{*}}=5.325 \mathrm{GeV} \text { for } f_{1}, f_{2}, f_{1}^{T}, f_{2}^{T} ; \widetilde{g}_{1}, \widetilde{g}_{2}, \widetilde{g}_{1}^{T}, \widetilde{g}_{2}^{T} \\
m_{B_{1}}=5.723 \mathrm{GeV} \text { for } g_{1}, g_{2}, g_{1}^{T}, g_{2}^{T} ; \widetilde{f}_{1}, \widetilde{f}_{2}, \widetilde{f}_{1}^{T}, \widetilde{f}_{2}^{T} \\
m_{B_{0}}=5.749 \mathrm{GeV} \text { for } f_{3} ; \widetilde{g}_{3} \\
m_{B}=5.280 \mathrm{GeV} \text { for } g_{3} ; \widetilde{f}_{3}
\end{array}\right.
$$

In Tables 1 and 2 we present the fit parameters $a_{0}, a_{1}$ and $a_{2}$ that results from our numerical analysis.

From the results for the form factors (see Tables 1 and 2), we observe that the LQSR-2 predictions nicely reproduce the relations among form factors given in Eq. (23). 
Fig. 3 Diagrams describing non-factorizable contributions to $\Lambda_{b} \rightarrow N^{*} \ell^{+} \ell^{-}$decay. Here, the black square corresponds to the four quark operators in the weak Hamiltonian and the crossed circle indicates possible radiation of the virtual photon
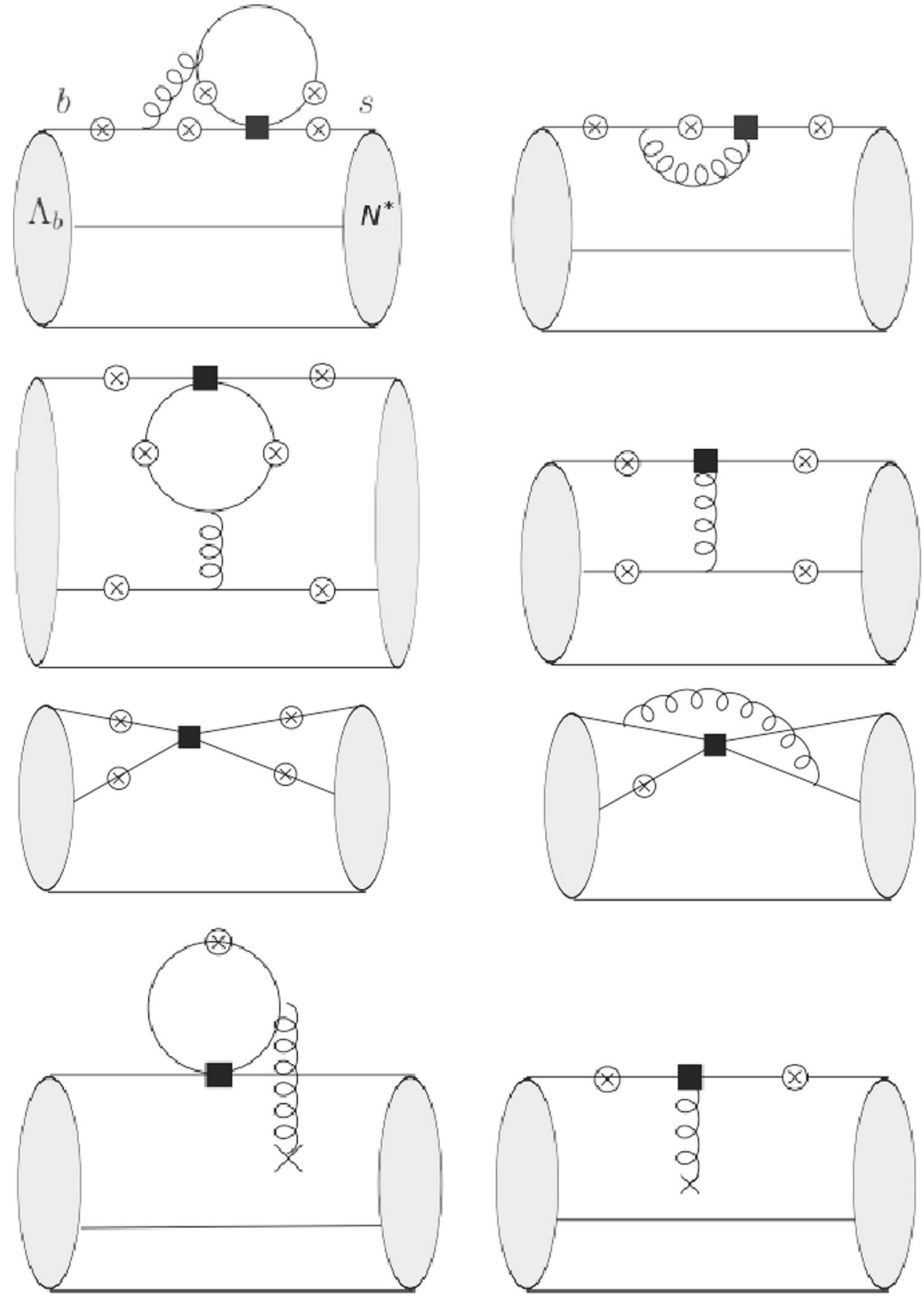

Using the definition of the form factors the differential decay width is calculated in the standard manner whose result is given below:

$\frac{d \Gamma(s)}{d s}=\frac{G^{2} \alpha^{2} m_{\Lambda_{b}}}{4096 \pi^{5}}\left|V_{t b} V_{t d}^{*}\right|^{2} v \sqrt{\lambda(1, r, s)}\left[T_{1}(s)+\frac{1}{3} T_{2}(s)\right]$,

where $v_{\ell}=\sqrt{1-4 m_{\ell}^{2} / q^{2}}$ is the lepton velocity, $\lambda(1, r, s)=$ $1+r^{2}+s^{2}-2 r-2 s-2 r s, s=q^{2} / m_{\Lambda_{b}}$, and $r=m_{N^{*}}^{2} / m_{\Lambda_{b}}^{2}$, $\alpha$ is the fine structural constant, and the expressions of $\Gamma_{1}(s)$ and $\Gamma_{2}(s)$ are given in the Appendix-C.

The dependence of the differential branching ratios on $q^{2}$ for the $\Lambda_{b} \rightarrow N^{*} \mu^{+} \mu^{-}$and $\Lambda_{b} \rightarrow N^{*} \tau^{+} \tau^{-}$decays, at $s_{0}=40 \mathrm{GeV}^{2}, M^{2}=25 \mathrm{GeV}^{2}$ and $\beta=-1$ are presented in Figs. 1 and 2, respectively. In these figures the graphical results predicted by the LCSR-1 and LCSR- 2 cases are shown together (Fig. 3).

Performing integration over $s$ in the range $4 m_{\ell}^{2} / m_{\Lambda_{b}}^{2} \leq$ $s \leq(1-\sqrt{r})^{2}$, we obtain the branching ratios for the 
Table 3 Branching ratios for the $\Lambda_{b} \rightarrow N^{*} \ell^{+} \ell^{-}$, $(\ell=e, \mu, \tau)$ decays

LCSR-1 LCSR-2

\begin{tabular}{lll}
\hline $\operatorname{Br}\left(\Lambda_{b} \rightarrow N^{*} e^{+} e^{-}\right)$ & $(4.62 \pm 1.85) \times 10^{-8}$ & $(3.56 \pm 1.42) \times 10^{-8}$ \\
$\operatorname{Br}\left(\Lambda_{b} \rightarrow N^{*} \mu^{+} \mu^{-}\right)$ & $(4.25 \pm 1.50) \times 10^{-8}$ & $(3.25 \pm 1.24) \times 10^{-8}$ \\
$\operatorname{Br}\left(\Lambda_{b} \rightarrow N^{*} \tau^{+} \tau^{-}\right)$ & $(0.25 \pm 0.09) \times 10^{-8}$ & $(0.180 \pm 0.067) \times 10^{-8}$ \\
\hline
\end{tabular}

$\Lambda_{b} \rightarrow N^{*} \ell^{+} \ell^{-}(\ell=e, \mu, \tau)$ decays in the case when long distance effects are due to the $J / \psi$ family, and these results are given in Table 3.

It follows from these results that, especially for the $e$ and $\mu$ channels, the branching ratios are quite large and could potentially be measurable at LHCb. The discovery of these decays would provide useful information about the inner structure of the $N^{*}$ baryon.

Finally, a comparison of the $\Lambda_{b} \rightarrow N \ell^{+} \ell^{-}$and $\Lambda_{b} \rightarrow$ $N^{*} \ell^{+} \ell^{-}$decays shows that the central values of the branching ratio of the former is approximately two (eight) times larger than the $\Lambda_{b} \rightarrow N^{*} e^{+} e^{-}\left(\Lambda_{b} \rightarrow N^{*} \tau^{+} \tau^{-}\right)$ decays.

\section{Conclusion}

In this work the transition form factors of the $\Lambda_{b} \rightarrow N^{*} \ell^{+} \ell^{-}$ decay are estimated, which is an alternative approach to extract information about the inner properties of the $N^{*}$ baryon. The contribution coming from the $\Lambda_{b}^{*}$ baryon is eliminated by constructing the sum rules with the choice of several different Lorentz structures. Using this result, we also calculate the branching ratio of the $\Lambda_{b} \rightarrow N \ell^{+} \ell^{-}$and decays. We see that the branching ratios of the $\Lambda_{b} \rightarrow N^{*} e^{+} e^{-}$and $\Lambda_{b} \rightarrow N^{*} \mu^{+} \mu^{-}$seem to be large enough to be detected at $\mathrm{LHCb}$.

Acknowledgements We sincerely thank Dr. M. Emmerich for providing us explicit forms of the DAs of the $N^{*}$ baryon. One of the authors, T. Barakat, thanks to the International Scientific Partnership Program ISPP at the King Saud University for funding his research work through ISPP no: 0038.

Data Availability Statement This manuscript has no associated data or the data will not be deposited. [Authors' comment: All the data that were used in the manuscript are available in the current literature.]

Open Access This article is distributed under the terms of the Creative Commons Attribution 4.0 International License (http://creativecomm ons.org/licenses/by/4.0/), which permits unrestricted use, distribution, and reproduction in any medium, provided you give appropriate credit to the original author(s) and the source, provide a link to the Creative Commons license, and indicate if changes were made.

Funded by SCOAP ${ }^{3}$.

\section{Appendix A: $N^{*}$ distribution amplitudes}

In this Appendix, we present the $N^{*}$ DAs, which are necessary to calculate the $\Lambda \rightarrow N^{*}$ transition form factors. The DAs of the $N^{*}$ baryon are defined from the matrix element $\left\langle 0\left|\epsilon^{a b c} u_{\alpha}^{a}\left(a_{1} x\right) d_{\beta}^{b}\left(a_{2} x\right) d_{\gamma}^{c}\left(a_{3} x\right)\right| N^{*}(p)\right\rangle$. The general decomposition of this matrix in terms of the DAs of the $N^{*}$ baryon is given below (see [12]).

$$
\begin{aligned}
& 4\left\langle 0\left|\epsilon^{a b c} u_{\alpha}^{a}\left(a_{1} x\right) d_{\beta}^{b}\left(a_{2} x\right) d_{\gamma}^{c}\left(a_{3} x\right)\right| N^{*}(p)\right\rangle \\
& =\mathcal{S}_{1} m_{N^{*}} C_{\alpha \beta} N_{\gamma}^{*}-\mathcal{S}_{2} m_{N^{*}}^{2} C_{\alpha \beta}\left(k N^{*}\right)_{\gamma} \\
& +\mathcal{P}_{1} m_{N^{*}}\left(\gamma_{5} C\right)_{\alpha \beta}\left(\gamma_{5} N^{*}\right)_{\gamma} \\
& +\mathcal{P}_{2} m_{N^{*}}^{2}\left(\gamma_{5} C\right)_{\alpha \beta}\left(\gamma_{5} k N^{*}\right)_{\gamma} \\
& -\left(\mathcal{V}_{1}+\frac{x^{2} m_{N^{*}}^{2}}{4} \mathcal{V}_{1}^{M}\right)(p C)_{\alpha \beta} N_{\gamma}^{*} \\
& +\mathcal{V}_{2} m_{N^{*}}(p C)_{\alpha \beta}\left(k N^{*}\right)_{\gamma} \\
& +\mathcal{V}_{3} m_{N^{*}}\left(\gamma_{\mu} C\right)_{\alpha \beta}\left(\gamma^{\mu} N^{*}\right)_{\gamma}-\mathcal{V}_{4} m_{N^{*}}^{2}(k C)_{\alpha \beta} N_{\gamma}^{*} \\
& -\mathcal{V}_{5} m_{N^{*}}^{2}\left(\gamma_{\mu} C\right)_{\alpha \beta}\left(i \sigma^{\mu \nu} x_{v} N^{*}\right)_{\gamma} \\
& +\mathcal{V}_{6} m_{N^{*}}^{3}(k C)_{\alpha \beta}\left(k N^{*}\right)_{\gamma} \\
& -\left(\mathcal{A}_{1}+\frac{x^{2} m_{N^{*}}^{2}}{4} \mathcal{A}_{1}^{M}\right)\left(p \gamma_{5} C\right)_{\alpha \beta}\left(\gamma N^{*}\right)_{\gamma} \\
& +\mathcal{A}_{2} m_{N^{*}}\left(p \gamma_{5} C\right)_{\alpha \beta}\left(k \gamma_{5} N^{*}\right)_{\gamma} \\
& +\mathcal{A}_{3} m_{N^{*}}\left(\gamma_{\mu} \gamma_{5} C\right)_{\alpha \beta}\left(\gamma^{\mu} \gamma_{5} N^{*}\right)_{\gamma} \\
& -\mathcal{A}_{4} m_{N^{*}}^{2}\left(k \gamma_{5} C\right)_{\alpha \beta}\left(\gamma_{5} N^{*}\right)_{\gamma} \\
& -\mathcal{A}_{5} m_{N^{*}}^{2}\left(\gamma_{\mu} \gamma_{5} C\right)_{\alpha \beta}\left(i \sigma^{\mu \nu} x_{\nu} \gamma_{5} N^{*}\right)_{\gamma} \\
& +\mathcal{A}_{6} m_{N^{*}}^{3}\left(k \gamma_{5} C\right)_{\alpha \beta}\left(k \gamma_{5} N^{*}\right)_{\gamma} \\
& -\left(\mathcal{T}_{1}+\frac{x^{2} m_{N^{*}}^{2}}{4} \mathcal{T}_{1}^{M}\right)\left(i \sigma_{\mu \nu} p_{\nu} C\right)_{\alpha \beta}\left(\gamma^{\mu} N^{*}\right)_{\gamma} \\
& +\mathcal{T}_{2} m_{N^{*}}\left(i \sigma_{\mu \nu} x^{\mu} p^{\nu} C\right)_{\alpha \beta} N_{\gamma}^{*} \\
& +\mathcal{T}_{3} m_{N^{*}}\left(\sigma_{\mu \nu} C\right)_{\alpha \beta}\left(\sigma^{\mu \nu} N^{*}\right)_{\gamma} \\
& +\mathcal{T}_{4} m_{N^{*}}\left(\sigma_{\mu \nu} p^{\nu} C\right)_{\alpha \beta}\left(\sigma^{\mu \rho} x_{\rho} N^{*}\right)_{\gamma} \\
& -\mathcal{T}_{5} m_{N^{*}}^{2}\left(i \sigma_{\mu \nu} x^{\nu} C\right)_{\alpha \beta}\left(\gamma^{\mu} N^{*}\right)_{\gamma} \\
& -\mathcal{T}_{6} m_{N^{*}}^{2}\left(i \sigma_{\mu \nu} x^{\mu} p^{\nu} C\right)_{\alpha \beta}\left(k N^{*}\right)_{\gamma} \\
& -\mathcal{T}_{7} m_{N^{*}}^{2}\left(\sigma_{\mu \nu} C\right)_{\alpha \beta}\left(\sigma^{\mu \nu} k N^{*}\right)_{\gamma} \\
& +\mathcal{T}_{8} m_{N^{*}}^{3}\left(\sigma_{\mu \nu} x^{\nu} C\right)_{\alpha \beta}\left(\sigma^{\mu \rho} x_{\rho} N^{*}\right)_{\gamma} .
\end{aligned}
$$

The functions labeled with calligraphic letters in the above expression do not possess definite twists but they can be written in terms of the $N^{*}$ distribution amplitudes (DAs) with definite and increasing twists via the scalar product $p \cdot x$ and the parameters $a_{i}, i=1,2,3$. The relations between the two sets of DAs for the $N^{*}$, and for the scalar, pseudo-scalar, vector, axial vector and tensor DAs for nucleons are: 


$$
\begin{aligned}
\mathcal{S}_{1} & =S_{1} \\
2(p \cdot x) \mathcal{S}_{2} & =S_{1}-S_{2} \\
\mathcal{P}_{1} & =P_{1} \\
2(p \cdot x) \mathcal{P}_{2} & =P_{2}-P_{1} \\
\mathcal{V}_{1} & =V_{1} \\
2(p \cdot x) \mathcal{V}_{2} & =V_{1}-V_{2}-V_{3} \\
2 V_{3} & =V_{3} \\
4(p \cdot x) \mathcal{V}_{4} & =-2 V_{1}+V_{3}+V_{4}+2 V_{5} \\
4(p \cdot x) \mathcal{V}_{5} & =V_{4}-V_{3} \\
4(p \cdot x)^{2} \mathcal{V}_{6} & =-V_{1}+V_{2}+V_{3}+V_{4}+V_{5}-V_{6} \\
\mathcal{A}_{1} & =A_{1} \\
2(p \cdot x) \mathcal{A}_{2} & =-A_{1}+A_{2}-A_{3} \\
2 \mathcal{A}_{3} & =A_{3} \\
4(p \cdot x) \mathcal{A}_{4} & =-2 A_{1}-A_{3}-A_{4}+2 A_{5} \\
4(p \cdot x) \mathcal{A}_{5} & =A_{3}-A_{4} \\
4(p \cdot x)^{2} \mathcal{A}_{6} & =A_{1}-A_{2}+A_{3}+A_{4}-A_{5}+A_{6} \\
\mathcal{T}_{1} & =T_{1} \\
2(p \cdot x) \mathcal{T}_{2} & =T_{1}+T_{2}-2 T_{3} \\
2 \mathcal{T}_{3} & =T_{7} \\
2(p \cdot x) \mathcal{T}_{4} & =T_{1}-T_{2}-2 T_{7} \\
2(p \cdot x) \mathcal{T}_{5} & =-T_{1}+T_{5}+2 T_{8} \\
4(p \cdot x)^{2} \mathcal{T}_{6} & =2 T_{2}-2 T_{3}-2 T_{4}+2 T_{5}+2 T_{7}+2 T_{8} \\
4(p \cdot x) \mathcal{T}_{7} & =T_{7}-T_{8} \\
4(p \cdot x)^{2} \mathcal{T}_{8} & =-T_{1}+T_{2}+T_{5}-T_{6}+2 T_{7}+2 T_{8}
\end{aligned}
$$

where the terms in $x^{2}, \mathcal{V}_{1}^{M}, \mathcal{A}_{1}^{M}$ and $\mathcal{T}_{1}^{M}$ are left aside.

The distribution amplitudes $F\left[a_{i}(p \cdot x)\right]=S_{i}, P_{i}, V_{i}, A_{i}$, $T_{i}$ can be represented as:

$$
\begin{aligned}
& F\left[a_{i}(p \cdot x)\right]=\int d x_{1} d x_{2} d x_{3} \delta\left(x_{1}+x_{2}\right. \\
& \left.\quad+x_{3}-1\right) e^{i p \cdot x \Sigma_{i} x_{i} a_{i}} F\left(x_{i}\right) .
\end{aligned}
$$

where, $x_{i}$ with $i=1,2$ and 3 are longitudinal momentum fractions carried by the participating quarks.

The explicit expressions for the $\Lambda$ DAs up to twist 6 are given as: Twist-3 DAs:

$$
\begin{aligned}
V_{1}\left(x_{i}, \mu\right)= & 120 x_{1} x_{2} x_{3}\left[\phi_{3}^{0}(\mu)+\phi_{3}^{+}(\mu)\left(1-3 x_{3}\right)\right], \\
A_{1}\left(x_{i}, \mu\right)= & 120 x_{1} x_{2} x_{3}\left(x_{2}-x_{1}\right) \phi_{3}^{-}(\mu), \\
T_{1}\left(x_{i}, \mu\right)= & 120 x_{1} x_{2} x_{3}\left[\phi_{3}^{0}(\mu)\right. \\
& \left.-\frac{1}{2}\left(\phi_{3}^{+}-\phi_{3}^{-}\right)(\mu)\left(1-3 x_{3}\right)\right] .
\end{aligned}
$$

Twist-4 DAs:

$$
\begin{aligned}
& V_{2}\left(x_{i}, \mu\right)=24 x_{1} x_{2}\left[\phi_{4}^{0}(\mu)+\phi_{4}^{+}(\mu)\left(1-5 x_{3}\right)\right], \\
& A_{2}\left(x_{i}, \mu\right)=24 x_{1} x_{2}\left(x_{2}-x_{1}\right) \phi_{4}^{-}(\mu),
\end{aligned}
$$

$$
\begin{aligned}
& T_{2}\left(x_{i}, \mu\right)=24 x_{1} x_{2}\left[\xi_{4}^{0}(\mu)+\xi_{4}^{+}(\mu)\left(1-5 x_{3}\right)\right], \\
& V_{3}\left(x_{i}, \mu\right)=12 x_{3}\left[\psi_{4}^{0}(\mu)\left(1-x_{3}\right)\right. \\
& +\psi_{4}^{+}(\mu)\left(1-x_{3}-10 x_{1} x_{2}\right) \\
& \left.+\psi_{4}^{-}(\mu)\left(x_{1}^{2}+x_{2}^{2}-x_{3}\left(1-x_{3}\right)\right)\right], \\
& A_{3}\left(x_{i}, \mu\right)=12 x_{3}\left(x_{2}-x_{1}\right)\left[\left(\psi_{4}^{0}\right.\right. \\
& \left.\left.+\psi_{4}^{+}\right)(\mu)+\psi_{4}^{-}(\mu)\left(1-2 x_{3}\right)\right], \\
& T_{3}\left(x_{i}, \mu\right)=6 x_{3}\left[\left(\phi_{4}^{0}+\psi_{4}^{0}+\xi_{4}^{0}\right)(\mu)\left(1-x_{3}\right)\right. \\
& +\left(\phi_{4}^{+}+\psi_{4}^{+}+\xi_{4}^{+}\right)(\mu)\left(1-x_{3}-10 x_{1} x_{2}\right) \\
& +\left(\phi_{4}^{-}-\psi_{4}^{-}+\xi_{4}^{-}\right)(\mu)\left(x_{1}^{2}\right. \\
& \left.\left.+x_{2}^{2}-x_{3}\left(1-x_{3}\right)\right)\right] \text {, } \\
& T_{7}\left(x_{i}, \mu\right)=6 x_{3}\left[\left(\phi_{4}^{0}+\psi_{4}^{0}-\xi_{4}^{0}\right)(\mu)\left(1-x_{3}\right)\right. \\
& +\left(\phi_{4}^{+}+\psi_{4}^{+}-\xi_{4}^{+}\right)(\mu)\left(1-x_{3}-10 x_{1} x_{2}\right) \\
& +\left(\phi_{4}^{-}-\psi_{4}^{-}-\xi_{4}^{-}\right)(\mu)\left(x_{1}^{2}+x_{2}^{2}\right. \\
& \left.\left.-x_{3}\left(1-x_{3}\right)\right)\right] \text {, } \\
& S_{1}\left(x_{i}, \mu\right)=6 x_{3}\left(x_{2}-x_{1}\right)\left[\left(\phi_{4}^{0}+\psi_{4}^{0}+\xi_{4}^{0}+\phi_{4}^{+}\right.\right. \\
& \left.+\psi_{4}^{+}+\xi_{4}^{+}\right)(\mu) \\
& \left.+\left(\phi_{4}^{-}-\psi_{4}^{-}+\xi_{4}^{-}\right)(\mu)\left(1-2 x_{3}\right)\right], \\
& P_{1}\left(x_{i}, \mu\right)=6 x_{3}\left(x_{1}-x_{2}\right)\left[\left(\phi_{4}^{0}+\psi_{4}^{0}-\xi_{4}^{0}+\phi_{4}^{+}\right.\right. \\
& \left.+\psi_{4}^{+}-\xi_{4}^{+}\right)(\mu) \\
& \left.+\left(\phi_{4}^{-}-\psi_{4}^{-}-\xi_{4}^{-}\right)(\mu)\left(1-2 x_{3}\right)\right] \text {. }
\end{aligned}
$$

Twist-5 DAs:

$$
\begin{aligned}
& V_{4}\left(x_{i}, \mu\right)=3\left[\psi_{5}^{0}(\mu)\left(1-x_{3}\right)+\psi_{5}^{+}(\mu)\left(1-x_{3}\right.\right. \\
&\left.-2\left(x_{1}^{2}+x_{2}^{2}\right)\right) \\
&\left.+\psi_{5}^{-}(\mu)\left(2 x_{1} x_{2}-x_{3}\left(1-x_{3}\right)\right)\right], \\
& A_{4}\left(x_{i}, \mu\right)=3\left(x_{2}-x_{1}\right) \\
& {\left[-\psi_{5}^{0}(\mu)+\psi_{5}^{+}(\mu)\left(1-2 x_{3}\right)+\psi_{5}^{-}(\mu) x_{3}\right], } \\
& T_{4}\left(x_{i}, \mu\right)= \frac{3}{2}\left[\left(\phi_{5}^{0}+\psi_{5}^{0}+\xi_{5}^{0}\right)(\mu)\left(1-x_{3}\right)\right. \\
&+\left(\phi_{5}^{+}+\psi_{5}^{+}+\xi_{5}^{+}\right)(\mu)\left(1-x_{3}-2\left(x_{1}^{2}+x_{2}^{2}\right)\right) \\
&\left.+\left(\phi_{5}^{-}-\psi_{5}^{-}+\xi_{5}^{-}\right)(\mu)\left(2 x_{1} x_{2}-x_{3}\left(1-x_{3}\right)\right)\right], \\
& 3 \\
& T_{8}\left(x_{i}, \mu\right)= \frac{2}{2}\left[\phi_{5}^{0}+\psi_{5}^{0}-\xi_{5}^{0}\right)(\mu)\left(1-x_{3}\right) \\
&+\left(\phi_{5}^{+}+\psi_{5}^{+}-\xi_{5}^{+}\right)(\mu)\left(1-x_{3}-2\left(x_{1}^{2}+x_{2}^{2}\right)\right) \\
&\left.+\left(\phi_{5}^{-}-\psi_{5}^{-}-\xi_{5}^{-}\right)(\mu)\left(2 x_{1} x_{2}-x_{3}\left(1-x_{3}\right)\right)\right], \\
& V_{5}\left(x_{i}, \mu\right)= 6 x_{3}\left[\phi_{5}^{0}(\mu)+\phi_{5}^{+}(\mu)\left(1-2 x_{3}\right)\right], \\
& A_{5}\left(x_{i}, \mu\right)= 6 x_{3}\left(x_{2}-x_{1}\right) \phi_{5}^{-}(\mu), \\
& T_{5}\left(x_{i}, \mu\right)= 6 x_{3}\left[\xi_{5}^{0}(\mu)+\xi_{5}^{+}(\mu)\left(1-2 x_{3}\right)\right],
\end{aligned}
$$




$$
\begin{aligned}
S_{2}\left(x_{i}, \mu\right)= & \frac{3}{2}\left(x_{2}-x_{1}\right)\left[-\left(\phi_{5}^{0}+\psi_{5}^{0}+\xi_{5}^{0}\right)(\mu)\right. \\
& +\left(\phi_{5}^{+}+\psi_{5}^{+}+\xi_{5}^{+}\right)(\mu)\left(1-2 x_{3}\right) \\
& \left.+\left(\phi_{5}^{-}-\psi_{5}^{-}+\xi_{5}^{-}\right)(\mu) x_{3}\right], \\
P_{2}\left(x_{i}, \mu\right)= & \frac{3}{2}\left(x_{2}-x_{1}\right)\left[-\left(-\phi_{5}^{0}-\psi_{5}^{0}+\xi_{5}^{0}\right)(\mu)\right. \\
& +\left(-\phi_{5}^{+}-\psi_{5}^{+}+\xi_{5}^{+}\right)(\mu)\left(1-2 x_{3}\right) \\
& \left.+\left(-\phi_{5}^{-}+\psi_{5}^{-}+\xi_{5}^{-}\right)(\mu) x_{3}\right] .
\end{aligned}
$$

Twist-6:

$$
\begin{aligned}
& V_{6}\left(x_{i}, \mu\right)=2\left[\phi_{6}^{0}(\mu)+\phi_{6}^{+}(\mu)\left(1-3 x_{3}\right)\right], \\
& A_{6}\left(x_{i}, \mu\right)=2\left(x_{2}-x_{1}\right) \phi_{6}^{-}, \\
& T_{6}\left(x_{i}, \mu\right)=2\left[\phi_{6}^{0}(\mu)-\frac{1}{2}\left(\phi_{6}^{+}-\phi_{6}^{-}\right)\left(1-3 x_{3}\right)\right] .
\end{aligned}
$$

Finally the $x^{2}$ corrections to the corresponding expressions $\mathcal{V}_{1}^{M}, \mathcal{A}_{1}^{M}, \mathcal{T}_{1}^{M}$ for the leading twist DAs $V_{1}, A_{1}$ and $T_{1}$ in the momentum fraction space are given as:

$$
\begin{aligned}
\mathcal{V}_{1}^{M}\left(x_{2}\right) & =\int_{0}^{1-x_{2}} d x_{1} V_{1}^{M}\left(x_{1}, x_{2}, 1-x_{1}-x_{2}\right) \\
& =\frac{x_{2}^{2}}{24}\left[f_{N^{*}} C_{f}^{u}\left(x_{2}\right)+\lambda_{1}^{N^{*}} C_{\lambda}^{u}\left(x_{2}\right)\right],
\end{aligned}
$$

where

$$
\begin{aligned}
C_{f}^{u}\left(x_{2}\right)= & \left(1-x_{2}\right)^{3}\left[113+495 x_{2}\right. \\
& -552 x_{2}^{2}-10 A_{1}^{u}\left(1-3 x_{2}\right) \\
& \left.+2 V_{1}^{d}\left(113-951 x_{2}+828 x_{2}^{2}\right)\right], \\
C_{\lambda}^{u}\left(x_{2}\right)= & -\left(1-x_{2}\right)^{3}\left[13-20 f_{1}^{d}+3 x_{2}+10 f_{1}^{u}\left(1-3 x_{2}\right)\right] .
\end{aligned}
$$

The expression for the axial-vector function $\mathcal{A}_{1}^{M(u)}\left(x_{2}\right)$ is given as:

$$
\begin{aligned}
\mathcal{A}_{1}^{M(u)}\left(x_{2}\right) & =\int_{0}^{1-x_{2}} d x_{1} A_{1}^{M}\left(x_{1}, x_{2}, 1-x_{1}-x_{2}\right), \\
& =\frac{x_{2}^{2}}{24}\left(1-x_{2}\right)^{3}\left[f_{N^{*}} D_{f}^{u}\left(x_{2}\right)+\lambda_{1}^{N^{*}} D_{\lambda}^{u}\left(x_{2}\right)\right],
\end{aligned}
$$

with

$$
\begin{aligned}
D_{f}^{u}\left(x_{2}\right)= & 11+45 x_{2}-2 A_{1}^{u}\left(113-951 x_{2}+828 x_{2}^{2}\right) \\
& +10 V_{1}^{d}\left(1-30 x_{2}\right), \\
D_{\lambda}^{u}\left(x_{2}\right)= & 29-45 x_{2}-10 f_{1}^{u}\left(7-9 x_{2}\right)-20 f_{1}^{d}\left(5-6 x_{2}\right) .
\end{aligned}
$$

Similarly, we get for the function $\mathcal{T}_{1}^{M(u)}\left(x_{2}\right)$ :

$$
\begin{aligned}
\mathcal{T}_{1}^{M(u)}\left(x_{2}\right) & =\int_{0}^{1-x_{2}} d x_{1} T_{1}^{M}\left(x_{1}, x_{2}, 1-x_{1}-x_{2}\right), \\
& =\frac{x^{2}}{48}\left[f_{N^{*}} E_{f}^{u}\left(x_{2}\right)+\lambda_{1}^{N^{*}} E_{\lambda}^{u}\left(x_{2}\right)\right],
\end{aligned}
$$

where

$$
\begin{aligned}
E_{f}^{u}\left(x_{2}\right)= & -\left\{( 1 - x _ { 2 } ) \left[3 \left(439+71 x_{2}\right.\right.\right. \\
& \left.-621 x_{2}^{2}+587 x_{2}^{3}-184 x_{2}^{4}\right) \\
& +4 A_{1}^{u}\left(1-x_{2}\right)^{2}\left(59-483 x_{2}+414 x_{2}^{2}\right) \\
& -4 V_{1}^{d}\left(1301-619 x_{2}-769 x_{2}^{2}\right. \\
& \left.\left.\left.+1161 x_{2}^{3}-414 x_{2}^{4}\right)\right]\right\} \\
& -12\left(73-220 V_{1}^{d}\right) \ln \left[x_{2}\right], \\
E_{\lambda}^{u}\left(x_{2}\right)= & -\left\{( 1 - x _ { 2 } ) \left[5-211 x_{2}+281 x_{2}^{2}\right.\right. \\
& -111 x_{2}^{3}+10\left(1+61 x_{2}-83 x_{2}^{2}+33 x_{2}^{3}\right) f_{1}^{d} \\
& \left.\left.-40\left(1-x_{2}\right)^{2}\left(2-3 x_{2}\right) f_{1}^{u}\right]\right\} \\
& -12\left(3-10 f_{1}^{d}\right) \ln \left[x_{2}\right] .
\end{aligned}
$$

The following functions are encountered to the above amplitudes and they can be defined in terms of the 8 independent parameters, namely $f_{N^{*}}, \lambda_{1}, \lambda_{2}$ and $f_{1}^{u}, f_{1}^{d}, f_{2}^{d}, A_{1}^{u}, V_{1}^{d}$ :

$$
\begin{aligned}
& \phi_{3}^{0}=\phi_{6}^{0}=f_{N^{*}} \\
& \phi_{4}^{0}=\phi_{5}^{0}=\frac{1}{2}\left(f_{N^{*}}+\lambda_{1}^{N^{*}}\right) \\
& \xi_{4}^{0}=\xi_{5}^{0}=\frac{1}{6} \lambda_{2}^{N^{*}} \\
& \psi_{4}^{0}=\psi_{5}^{0}=\frac{1}{2}\left(f_{N^{*}}-\lambda_{1}^{N^{*}}\right), \\
& \phi_{3}^{-}=\frac{21}{2} f_{N^{*}} A_{1}^{u}, \quad \phi_{3}^{+}=\frac{7}{2} f_{N^{*}}\left(1-V_{1}^{d}\right), \\
& \phi_{4}^{+}=\frac{1}{4}\left[f_{N^{*}}\left(3-10 V_{1}^{d}\right)+\lambda_{1}^{N^{*}}\left(3-10 f_{1}^{d}\right)\right] \text {, } \\
& \phi_{4}^{-}=-\frac{5}{4}\left[f_{N^{*}}\left(1-2 A_{1}^{u}\right)-\lambda_{1}^{N^{*}}\left(1-2 f_{1}^{d}-4 f_{1}^{u}\right)\right] \text {, } \\
& \psi_{4}^{+}=-\frac{1}{4}\left[f_{N^{*}}\left(2+5 A_{1}^{u}-5 V_{1}^{d}\right)\right. \\
& \left.-\lambda_{1}^{N^{*}}\left(2-5 f_{1}^{d}-5 f_{1}^{u}\right)\right] \text {, } \\
& \psi_{4}^{-}=\frac{5}{4}\left[f_{N^{*}}\left(2-A_{1}^{u}-3 V_{1}^{d}\right)-\lambda_{1}^{N^{*}}\left(2-7 f_{1}^{d}+f_{1}^{u}\right)\right] \text {, } \\
& \xi_{4}^{+}=\frac{1}{16} \lambda_{2}^{N^{*}}\left(4-15 f_{2}^{d}\right), \\
& \xi_{4}^{-}=\frac{5}{16} \lambda_{2}^{N^{*}}\left(4-15 f_{2}^{d}\right), \\
& \phi_{5}^{+}=-\frac{5}{6}\left[f_{N^{*}}\left(3+4 V_{1}^{d}\right)-\lambda_{1}^{N^{*}}\left(1-4 f_{1}^{d}\right)\right] \text {, } \\
& \phi_{5}^{-}=-\frac{5}{3}\left[f_{N^{*}}\left(1-2 A_{1}^{u}\right)-\lambda_{1}^{N^{*}}\left(f_{1}^{d}-f_{1}^{u}\right)\right] \text {, } \\
& \psi_{5}^{+}=-\frac{5}{6}\left[f_{N^{*}}\left(5+2 A_{1}^{u}-2 V_{1}^{d}\right)-\lambda_{1}^{N^{*}}\left(1-2 f_{1}^{d}-2 f_{1}^{u}\right)\right] \text {, } \\
& \psi_{5}^{-}=\frac{5}{3}\left[f_{N^{*}}\left(2-A_{1}^{u}-3 V_{1}^{d}\right)+\lambda_{1}^{N^{*}}\left(f_{1}^{d}-f_{1}^{u}\right)\right] \text {, } \\
& \xi_{5}^{+}=\frac{5}{36} \lambda_{2}^{N^{*}}\left(2-9 f_{2}^{d}\right),
\end{aligned}
$$


Table 4 Parameters of the DAs for the $N^{*}(1535)$ baryon at $\mu^{2}=2 \mathrm{GeV}^{2}$

\begin{tabular}{lllllllllll}
\hline & Model & $\lambda_{1}^{N^{*}} / \lambda_{1}^{N}$ & $f_{N^{*}} / \lambda_{1}^{N^{*}}$ & $\varphi_{10}$ & $\varphi_{11}$ & $\varphi_{20}$ & $\varphi_{21}$ & $\varphi_{22}$ & $\eta_{10}$ & $\eta_{11}$ \\
\hline$[17]$ & LCSR-1 & 0.633 & 0.027 & 0.36 & -0.95 & 0 & 0 & 0 & 0 \\
{$[17]$} & LCSR-2 & 0.633 & 0.027 & 0.37 & -0.96 & 0 & 0 & 0 & -0.29 & 0.23 \\
{$[18]$} & LATTICE & 0.633 & 0.027 & 0.28 & -0.86 & 1.7 & -2 & 1.7 & 0 & 0 \\
\hline
\end{tabular}

$\xi_{5}^{-}=-\frac{5}{4} \lambda_{2}^{N^{*}} f_{2}^{d}$,

$\phi_{6}^{+}=\frac{1}{2}\left[f_{N^{*}}\left(1-4 V_{1}^{d}\right)-\lambda_{1}^{N^{*}}\left(1-2 f_{1}^{d}\right)\right]$,

$\phi_{6}^{-}=\frac{1}{2}\left[f_{N^{*}}\left(1+4 A_{1}^{d}\right)+\lambda_{1}^{N^{*}}\left(1-4 f_{1}^{d}-2 f_{1}^{u}\right)\right]$,

where the parameters $A_{1}^{u}, V_{1}^{d}, f_{1}^{d}, f_{1}^{u}$ and $f_{2}^{d}$ are defined as [17],

$A_{1}^{u}=\varphi_{10}+\varphi_{11}$,

$V_{1}^{d}=\frac{1}{3}-\varphi_{10}+\frac{1}{3} \varphi_{11}$,

$f_{1}^{u}=\frac{1}{10}-\frac{1}{6} \frac{f_{N^{*}}}{\lambda_{1}^{N^{*}}}-\frac{3}{5} \eta_{10}-\frac{1}{3} \eta_{11}$,

$f_{1}^{d}=\frac{3}{10}-\frac{1}{6} \frac{f_{N^{*}}}{\lambda_{1}^{N^{*}}}+\frac{1}{5} \eta_{10}-\frac{1}{3} \eta_{11}$,

$f_{2}^{d}=\frac{4}{15}+\frac{2}{5} \xi_{10}$

The numerical values of the parameters $\varphi_{10}, \varphi_{11}, \varphi_{20}, \varphi_{21}$, $\varphi_{22}, \eta_{10}, \eta_{11}$ and $f_{N^{*}} / \lambda_{1}^{N^{*}}$, and $\lambda_{1}^{N^{*}} / \lambda_{1}^{N}$ are presented in Table 4 (this table is taken from [10]).

\section{Appendix B}

\section{Invariant functions of the transition current $\left(\bar{b} \gamma_{\mu} d\right)$}

In this section we present the expressions for the invariant functions $\Pi_{1}^{I}(p, q), \Pi_{2}^{I}(p, q), \Pi_{3}^{I}(p, q) \Pi_{4}^{I}(p, q), \Pi_{5}^{I}(p, q)$, and $\Pi_{6}^{I}(p, q)$ for coefficients of the structures $p_{\mu} \gamma_{5}, p_{\mu} q \gamma_{5}$, $\gamma_{\mu} \gamma_{5}, \gamma_{\mu} q \gamma_{5}, q_{\mu} \gamma_{5}$, and $q_{\mu} q \gamma_{5}$, respectively, for the transition current $\left(\bar{b} \gamma_{\mu} d\right)$ which appear in the sum rules.

Invariant function $\Pi_{1}^{I}(p, q)$ for the structure $p_{\mu} \gamma_{5}$

$$
\begin{aligned}
\mathcal{D}_{13}^{I}(x)= & -m_{N^{*}}^{2}\left\{x m_{N^{*}}\left(x^{2} m_{N^{*}}^{2}+Q^{2}\right)(2+\beta) \widetilde{\widetilde{\mathcal{T}}}_{6}\right. \\
& +m_{b}\left[\widetilde{\mathcal{\mathcal { A }}}_{6} x^{2} m_{N^{*}}^{2}(1+3 \beta)+Q^{2}(-1+\beta) \widetilde{\widetilde{\mathcal{T}}}_{6}\right. \\
& +x^{2} m_{N^{*}}^{2}\left(\widetilde{\widetilde{\mathcal{V}}}_{6}(3+\beta)+2(-1+\beta) \widetilde{\widetilde{\mathcal{T}}}_{8}\right) \\
& +x m_{N^{*}}\left(\widetilde{\widetilde{\mathcal{A}}}_{6}-3 \widetilde{\widetilde{\mathcal{V}}}_{6}(-1+\beta)\right. \\
& -\widetilde{\widetilde{\mathcal{A}}}_{6} \beta-(2+\beta) \widetilde{\widetilde{\mathcal{T}}}_{6}
\end{aligned}
$$

$$
\begin{aligned}
& \left.\left.\left.+2(5+4 \beta) \widetilde{\widetilde{\mathcal{T}}}_{8}\right) m_{b}\right]\right\}
\end{aligned}
$$

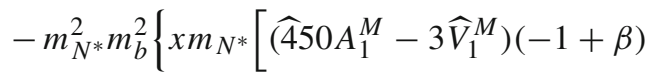

$$
\begin{aligned}
& \left.+2(5+4 \beta) \widehat{T}_{1}^{M}\right] \\
& +\left(\widehat{V}_{1}^{M}(3+\beta)-\widehat{A}_{1}^{M}(1+3 \beta)\right. \\
& \left.\left.+2(-1+\beta) \widehat{T}_{1}^{M}\right) m_{b}\right\} \text {. } \\
& \mathcal{D}_{12}^{I}(x)=\frac{m_{N^{*}}^{2}}{2}\left\{x m _ { N ^ { * } } \left[\widetilde{\widetilde{\mathcal{A}}}_{6}(-1+\beta)\right.\right. \\
& +3 \widetilde{\widetilde{V}}_{6}(-1+\beta)+2(2+\beta) \widetilde{\widetilde{\mathcal{T}}}_{6} \\
& \left.\left.-2(5+4 \beta) \widetilde{\widetilde{\mathcal{T}}}_{8}\right]+(-1+\beta) \widetilde{\widetilde{\mathcal{T}}}_{6} m_{b}\right\} \\
& +\frac{m_{N^{*}}}{4}\left\{-3 x^{2} m_{N^{*}}^{2} \tilde{\mathcal{V}}_{4}+7 x^{2} m_{N^{*}}^{2} \tilde{\mathcal{V}}_{5}\right. \\
& -4 x^{2} m_{N^{*}}^{2} \widetilde{\mathcal{P}}_{2}+4\left(\widetilde{\mathcal{A}}_{2}+\widetilde{\mathcal{V}}_{2}\right) Q^{2}+8 x^{2} m_{N^{*}}^{2} \widetilde{\mathcal{S}}_{2} \\
& -\widetilde{\mathcal{A}}_{4} x^{2} m_{N^{*}}^{2}(-1+\beta)+5 \widetilde{\mathcal{A}}_{5} x^{2} m_{N^{*}}^{2}(-1+\beta) \\
& +3 x^{2} m_{N^{*}}^{2} \widetilde{\mathcal{V}}_{4} \beta-7 x^{2} m_{N^{*}}^{2} \widetilde{\mathcal{V}}_{5} \beta \\
& -8 x^{2} m_{N^{*}}^{2} \widetilde{\mathcal{P}}_{2} \beta \\
& -4 \widetilde{\mathcal{A}}_{2} Q^{2} \beta-4 \widetilde{\mathcal{V}}_{2} Q^{2} \beta+4 x^{2} m_{N^{*}}^{2} \widetilde{\mathcal{S}}_{2} \beta \\
& -4 x^{2} m_{N^{*}}^{2} \widetilde{\mathcal{T}}_{2}+2 Q^{2} \widetilde{\mathcal{T}}_{2} \\
& -2 x^{2} m_{N^{*}}^{2} \beta \widetilde{\mathcal{T}}_{2}+4 Q^{2} \beta \widetilde{\mathcal{T}}_{2}+4 x^{2} m_{N^{*}}^{2} \widetilde{\mathcal{T}}_{4} \\
& +14 Q^{2} \widetilde{\mathcal{T}}_{4}+2 x^{2} m_{N^{*}}^{2} \beta \widetilde{\mathcal{T}}_{4}+16 Q^{2} \beta \widetilde{\mathcal{T}}_{4} \\
& -20 x^{2} m_{N^{*}}^{2} \widetilde{\mathcal{T}}_{5}-16 x^{2} m_{N^{*}}^{2} \beta \widetilde{\mathcal{T}}_{5} \\
& -36 x^{2} m_{N^{*}}^{2}(1+\beta) \widetilde{\mathcal{T}}_{7} \\
& +m_{b}\left[x m _ { N ^ { * } } \left(\widetilde{\mathcal{A}}_{4}+\widetilde{\mathcal{A}}_{5}+6 \widetilde{\mathcal{V}}_{2}\right.\right. \\
& -3 \widetilde{\mathcal{V}}_{4}-3 \widetilde{\mathcal{V}}_{5}+\widetilde{\mathcal{A}}_{2}(2+6 \beta)-4 \widetilde{\mathcal{T}}_{4} \\
& +\beta\left(3 \tilde{\mathcal{A}}_{4}+3 \tilde{\mathcal{A}}_{5}+2 \tilde{\mathcal{V}}_{2}-\tilde{\mathcal{V}}_{4}-\tilde{\mathcal{V}}_{5}+4 \widetilde{\mathcal{T}}_{4}-4 \widetilde{\mathcal{T}}_{5}\right) \\
& \left.+4 \widetilde{\mathcal{T}}_{5}\right)+2\left(\widetilde{\mathcal{A}}_{2}+3 \widetilde{\mathcal{V}}_{2}-\widetilde{\mathcal{A}}_{2} \beta+2 \widetilde{\mathcal{T}}_{2}+8 \widetilde{\mathcal{T}}_{4}\right. \\
& \left.\left.\left.+\beta\left(-3 \widetilde{\mathcal{V}}_{2}+\widetilde{\mathcal{T}}_{2}+7 \widetilde{\mathcal{T}}_{4}\right)\right) m_{b}\right]\right\} \\
& +\frac{x m_{N^{*}}^{3}}{2}\left[\widehat{A}_{1}^{M}+3 \widehat{V}_{1}^{M}(-1+\beta)-\widehat{A}_{1}^{M} \beta\right. \\
& \left.-2(5+4 \beta) \widehat{T}_{1}^{M}\right] \text {. } \\
& \mathcal{D}_{11}^{I}(x)=\frac{m_{N^{*}}}{2}(-1+\beta)\left(\widetilde{\mathcal{A}}_{2}-\widetilde{\mathcal{V}}_{2}-\widetilde{\mathcal{T}}_{2}-\widetilde{\mathcal{T}}_{4}\right) \\
& +\frac{x m_{N^{*}}}{2}\left\{-3 \widehat{\mathcal{A}}_{3}+3 \widehat{\mathcal{V}}_{1}-5 \widehat{\mathcal{V}}_{3}+\widehat{\mathcal{A}}_{1}(-1+\beta)\right. \\
& +\beta\left(3 \widehat{\mathcal{A}}_{3}-3 \widehat{\mathcal{V}}_{1}+5 \widehat{\mathcal{V}}_{3}+4 \widehat{\mathcal{P}}_{1}+2 \widehat{\mathcal{S}}_{1}\right.
\end{aligned}
$$




$$
\begin{aligned}
& \left.\left.+8 \widehat{\mathcal{T}}_{1}-18 \widehat{\mathcal{T}}_{3}\right)+2\left(\widehat{\mathcal{P}}_{1}+2 \widehat{\mathcal{S}}_{1}+5 \widehat{\mathcal{T}}_{1}-9 \widehat{\mathcal{T}}_{3}\right)\right\} \\
& +\left(\widehat{\mathcal{V}}_{1}(3+\beta)-\widehat{\mathcal{A}}_{1}(1+3 \beta)+2(-1+\beta) \widehat{\mathcal{T}}_{1}\right) m_{b} .
\end{aligned}
$$

Invariant function $\Pi_{2}^{I}(p, q)$ for the structure $p_{\mu} q \gamma_{5}$

$$
\begin{aligned}
& \mathcal{D}_{22}^{I}(x)=-m_{N^{*}}^{2}\left\{\left(x^{2} m_{N^{*}}^{2}+Q^{2}\right)(2+\beta) \widetilde{\widetilde{T}}_{6}\right. \\
& +x m_{N^{*}}\left[\widetilde{\widetilde{\mathcal{A}}}_{6}+3 \widetilde{\widetilde{\mathcal{A}}}_{6} \beta+\widetilde{\widetilde{V}}_{6}(3+\beta)\right. \\
& \left.-(-1+\beta)\left(\widetilde{\widetilde{\mathcal{T}}}_{6}-2 \widetilde{\widetilde{\mathcal{T}}}_{8}\right)\right] \\
& \left.m_{b}+\left(\widetilde{\widetilde{\mathcal{T}}}_{6}+2 \beta \widetilde{\widetilde{\mathcal{T}}}_{6}\right) m_{b}^{2}\right\} \\
& +m_{N^{*}}^{2}(-1+\beta)\left(\widehat{A}_{1}^{M}+\widehat{V}_{1}^{M}+2 \widehat{T}_{1}^{M}\right) m_{b}^{2} \text {. } \\
& \mathcal{D}_{22}^{I}(x)=\frac{3 m_{N^{*}}^{2}}{2} \widetilde{\widetilde{\mathcal{T}}}_{6} \\
& -\frac{m_{N^{*}}}{4}\left\{x m _ { N ^ { * } } \left[-\widetilde{\mathcal{A}}_{4}+5 \widetilde{\mathcal{A}}_{5}+4 \widetilde{\mathcal{V}}_{2}\right.\right. \\
& +3 \widetilde{\mathcal{V}}_{4}-7 \widetilde{\mathcal{V}}_{5}+4 \widetilde{\mathcal{P}}_{2} \\
& -8 \widetilde{\mathcal{S}}_{2}-4 \widetilde{\mathcal{A}}_{2}(-1+\beta)+6 \widetilde{\mathcal{T}}_{2}+10 \widetilde{\mathcal{T}}_{4} \\
& +20 \widetilde{\mathcal{T}}_{5}+36 \widetilde{\mathcal{T}}_{7}+\beta\left(\widetilde{\mathcal{A}}_{4}-5 \widetilde{\mathcal{A}}_{5}-4 \widetilde{\mathcal{V}}_{2}-3 \widetilde{\mathcal{V}}_{4}\right. \\
& +7 \widetilde{\mathcal{V}}_{5}+8 \widetilde{\mathcal{P}}_{2}-4 \widetilde{\mathcal{S}}_{2}+6 \widetilde{\mathcal{I}}_{2}+14 \widetilde{\mathcal{T}}_{4} \\
& \left.\left.+16 \widetilde{\mathcal{T}}_{5}+36 \widetilde{\mathcal{T}}_{7}\right)\right]-2\left(\widetilde{\mathcal{A}}_{2}+3 \tilde{\mathcal{A}}_{2} \beta+\tilde{\mathcal{V}}_{2}(3+\beta)\right. \\
& \left.\left.+(-1+\beta)\left(\widetilde{\mathcal{T}}_{2}+\widetilde{\mathcal{T}}_{4}\right)\right) m_{b}\right\} \\
& +\frac{m_{N^{*}}^{2}}{2}(-1+\beta)\left(\widehat{A}_{1}^{M}+\widehat{V}_{1}^{M}+2 \widehat{T}_{1}^{M}\right) \text {. }
\end{aligned}
$$

Invariant function $\Pi_{3}^{I}(p, q)$ for the structure $\gamma_{\mu} \gamma_{5}$

$$
\begin{aligned}
\mathcal{D}_{33}^{I}(x)= & m_{N^{*}}^{2} 4 x m_{b}\left\{-3 x m_{N^{*}}\left(x^{2} m_{N^{*}}^{2}\right.\right. \\
& \left.+Q^{2}\right)(-1+\beta) \widetilde{\widetilde{\mathcal{T}}}_{6}+m_{b}\left[2 \widetilde{\widetilde{\mathcal{A}}}_{6} x^{2} m_{N^{*}}^{2}(-1+\beta)\right. \\
& -3 Q^{2}(1+\beta) \widetilde{\widetilde{\mathcal{T}}}_{6}+k x^{2} m_{N^{*}}^{2}\left(6 \widetilde{\widetilde{\mathcal{V}}}_{6}(-1+\beta)\right. \\
& \left.+3(1+\beta) \widetilde{\widetilde{\mathcal{T}}}_{6}-2(11+7 \beta) \widetilde{\widetilde{\mathcal{T}}}_{8}\right) \\
& +m_{b}\left(x m _ { N ^ { * } } \left[2 \widetilde{\widetilde{\mathcal{A}}}_{6}(1+\beta)\right.\right. \\
& \left.+2 \widetilde{\widetilde{V}}_{6}(1+\beta)+(-1+\beta)\left(3 \widetilde{\widetilde{\mathcal{T}}}_{6}-14 \widetilde{\widetilde{\mathcal{T}}}_{8}\right)\right] \\
& \left.\left.\left.-3(1+\beta) \widetilde{\widetilde{\mathcal{T}}}_{6} m_{b}\right)\right]\right\} \\
& +m_{N^{*}}^{2} m_{b}^{2}\left\{-\left[\left(2 x^{2} m_{N^{*}}^{2}\left(\widehat{A}_{1}^{M}-\widehat{V}_{1}^{M}\right)\right.\right.\right. \\
& \left.\left.-\left(\widehat{A}_{1}^{M}+\widehat{V}_{1}^{M}\right) Q^{2}\right)(-1+\beta)\right] \\
& -\left[-2 Q^{2}(-1+\beta)+9 x^{2} m_{N^{*}}^{2}(1+\beta)\right] \widehat{T}_{1}^{M} \\
& +x m_{N^{*}}\left[\left(-\widehat{A}_{1}^{M}+\widehat{V}_{1}^{M}\right)(1+\beta)\right. \\
& \left.-7(-1+\beta) \widehat{T}_{1}^{M}\right] m_{b}
\end{aligned}
$$

$$
\begin{aligned}
& \left.+(-1+\beta)\left(\widehat{A}_{1}^{M}+\widehat{V}_{1}^{M}+2 \widehat{T}_{1}^{M}\right) m_{b}^{2}\right\} . \\
& \mathcal{D}_{32}^{I}(x)=\frac{m_{N^{*}}^{2}}{8 x}\left\{2 \widetilde{\widetilde{\mathcal{A}}}_{6} x^{2} m_{N^{*}}^{2}(-1+\beta)-Q^{2}(-1+\beta) \widetilde{\widetilde{\mathcal{T}}}_{6}\right. \\
& +x^{2} m_{N^{*}}^{2}\left[6 \widetilde{\widetilde{V}}_{6}(-1+\beta)+(7+5 \beta) \widetilde{\widetilde{\mathcal{T}}}_{6}\right. \\
& \left.-2(11+7 \beta) \widetilde{\widetilde{T}}_{8}\right] \\
& +m_{b}\left[x m _ { N ^ { * } } \left(2 \widetilde{\widetilde{V}}_{6}(3+\beta)+\widetilde{\widetilde{\mathcal{A}}}_{6}(2+6 \beta)\right.\right. \\
& \left.\left.\left.+(-1+\beta)\left(\widetilde{\widetilde{\mathcal{T}}}_{6}+4 \widetilde{\widetilde{\mathcal{T}}}_{8}\right)\right)+(7+5 \beta) \widetilde{\widetilde{\mathcal{T}}}_{6} m_{b}\right]\right\} \\
& +\frac{m_{N^{*}}}{8 x}\left\{-\left[x m _ { N ^ { * } } ( x ^ { 2 } m _ { N ^ { * } } ^ { 2 } + Q ^ { 2 } ) \left(4 \tilde{\mathcal{A}}_{2}(-1+\beta)\right.\right.\right. \\
& \left.\left.+4 \widetilde{\mathcal{V}}_{2}(-1+\beta)-3(1+\beta)\left(\widetilde{\mathcal{T}}_{2}+5 \widetilde{\mathcal{T}}_{4}\right)\right)\right] \\
& +m_{b}\left[x^{2} m_{N^{*}}^{2} \tilde{\mathcal{V}}_{4}-9 x^{2} m_{N^{*}}^{2} \tilde{\mathcal{V}}_{5}+6 x^{2} m_{N^{*}}^{2} \widetilde{\mathcal{P}}_{2}\right. \\
& -2\left(\widetilde{\mathcal{A}}_{2}+\widetilde{\mathcal{V}}_{2}\right) Q^{2}-2 x^{2} m_{N^{*}}^{2} \widetilde{\mathcal{S}}_{2}+x^{2} m_{N^{*}}^{2} \widetilde{\mathcal{V}}_{4} \beta \\
& -5 x^{2} m_{N^{*}}^{2} \widetilde{\mathcal{V}}_{5} \beta-6 x^{2} m_{N^{*}}^{2} \widetilde{\mathcal{P}}_{2} \beta \\
& -2 \widetilde{\mathcal{A}}_{2} Q^{2} \beta-2 \widetilde{\mathcal{V}}_{2} Q^{2} \beta+2 x^{2} m_{N^{*}}^{2} \widetilde{\mathcal{S}}_{2} \beta \\
& -\widetilde{\mathcal{A}}_{4} x^{2} m_{N^{*}}^{2}(1+\beta) \\
& +\widetilde{\mathcal{A}}_{5} x^{2} m_{N^{*}}^{2}(5+9 \beta)+3 x^{2} m_{N^{*}}^{2} \widetilde{\mathcal{T}}_{2} \\
& -3 Q^{2} \widetilde{\mathcal{T}}_{2}-3 x^{2} m_{N^{*}}^{2} \beta \widetilde{\mathcal{T}}_{2}+3 Q^{2} \beta \widetilde{\mathcal{T}}_{2}-3 x^{2} m_{N^{*}}^{2} \widetilde{\mathcal{T}}_{4} \\
& -11 Q^{2} \widetilde{\mathcal{T}}_{4}+3 x^{2} m_{N^{*}}^{2} \beta \widetilde{\mathcal{T}}_{4}+11 Q^{2} \beta \widetilde{\mathcal{T}}_{4} \\
& +14 x^{2} m_{N^{*}}^{2} \widetilde{\mathcal{T}}_{5} \\
& -14 x^{2} m_{N^{*}}^{2} \beta \widetilde{\mathcal{T}}_{5}-24 x^{2} m_{N^{*}}^{2}(-1+\beta) \widetilde{\mathcal{T}}_{7} \\
& +m_{b}\left(x m _ { N ^ { * } } \left[\tilde{\mathcal{A}}_{4}-7 \tilde{\mathcal{A}}_{5}+2 \tilde{\mathcal{V}}_{2}-3 \tilde{\mathcal{V}}_{4}+5 \tilde{\mathcal{V}}_{5}\right.\right. \\
& -6 \widetilde{\mathcal{P}}_{2}+6 \widetilde{\mathcal{S}}_{2}+2 \tilde{\mathcal{A}}_{2}(-1+\beta)-3 \widetilde{\mathcal{T}}_{2}+7 \widetilde{\mathcal{T}}_{4} \\
& -22 \widetilde{\mathcal{T}}_{5}-36 \widetilde{\mathcal{T}}_{7}-\beta\left(\widetilde{\mathcal{A}}_{4}-7 \tilde{\mathcal{A}}_{5}+2 \widetilde{\mathcal{V}}_{2}-3 \widetilde{\mathcal{V}}_{4}\right. \\
& \left.\left.+5 \widetilde{\mathcal{V}}_{5}+6 \widetilde{\mathcal{P}}_{2}-6 \widetilde{\mathcal{S}}_{2}+3 \widetilde{\mathcal{T}}_{2}+\widetilde{\mathcal{T}}_{4}+14 \widetilde{\mathcal{T}}_{5}+36 \widetilde{\mathcal{T}}_{7}\right)\right] \\
& -\left(2 \widetilde{\mathcal{A}}_{2}(1+\beta)+2 \widetilde{\mathcal{V}}_{2}(1+\beta)\right. \\
& \left.\left.\left.\left.-(-1+\beta)\left(3 \widetilde{\mathcal{T}}_{2}+11 \widetilde{\mathcal{T}}_{4}\right)\right) m_{b}\right)\right]\right\} \\
& +\frac{m_{N^{*}}^{2}}{4 x}\left\{-\left[\left(2 x^{2} m_{N^{*}}^{2}\left(\widehat{A}_{1}^{M}-\widehat{V}_{1}^{M}\right)\right.\right.\right. \\
& \left.\left.-\left(\widehat{A}_{1}^{M}+\widehat{V}_{1}^{M}\right) Q^{2}\right)(-1+\beta)\right] \\
& -\left[-2 Q^{2}(-1+\beta)+9 x^{2} m_{N^{*}}^{2}(1+\beta)\right] \widehat{T}_{1}^{M} \\
& \left.-(-1+\beta)\left(\widehat{A}_{1}^{M}+\widehat{V}_{1}^{M}+2 \widehat{T}_{1}^{M}\right) m_{b}^{2}\right\} . \\
& \mathcal{D}_{31}^{I}(x)=\frac{m_{N^{*}}^{2}}{8 x}(-1+\beta) \widetilde{\widetilde{T}}_{6}+\frac{m_{N^{*}}}{8 x}\left\{2 x m_{N^{*}}(-1+\beta)\right. \\
& {\left[\tilde{\mathcal{A}}_{2}+\tilde{\mathcal{A}}_{5}-\tilde{\mathcal{V}}_{2}+\tilde{\mathcal{V}}_{5}+\widetilde{\mathcal{P}}_{2}+\tilde{\mathcal{S}}_{2}-\widetilde{\mathcal{T}}_{4}+\widetilde{\mathcal{T}}_{5}\right]} \\
& +\left[2 \widetilde{\mathcal{A}}_{2}(1+\beta)+2 \widetilde{\mathcal{V}}_{2}(1+\beta)\right. \\
& \left.\left.-(-1+\beta)\left(3 \widetilde{\mathcal{T}}_{2}+11 \widetilde{\mathcal{T}}_{4}\right)\right] m_{b}\right\} \\
& +\frac{1}{4 x}\left\{2 x^{2} m_{N^{*}}^{2} \widehat{\mathcal{V}}_{1}-4 x^{2} m_{N^{*}}^{2} \widehat{\mathcal{V}}_{3}\right.
\end{aligned}
$$




$$
\begin{aligned}
& +3 x^{2} m_{N^{*}}^{2} \widehat{\mathcal{P}}_{1}+\widehat{\mathcal{V}}_{1} Q^{2}+3 x^{2} m_{N^{*}}^{2} \widehat{\mathcal{S}}_{1} \\
& +4 \widehat{\mathcal{A}}_{3} x^{2} m_{N^{*}}^{2}(-1+\beta) \\
& +\widehat{\mathcal{A}}_{1}\left(2 x^{2} m_{N^{*}}^{2}-Q^{2}\right)(-1+\beta)-2 x^{2} m_{N^{*}}^{2} \widehat{\mathcal{V}}_{1} \beta \\
& +4 x^{2} m_{N^{*}}^{2} \widehat{\mathcal{V}}_{3} \beta+3 x^{2} m_{N^{*}}^{2} \widehat{\mathcal{P}}_{1} \beta-\widehat{\mathcal{V}}_{1} Q^{2} \beta \\
& +3 x^{2} m_{N^{*}}^{2} \widehat{\mathcal{S}}_{1} \beta+9 x^{2} m_{N^{*}}^{2} \widehat{\mathcal{T}}_{1}+2 Q^{2} \widehat{\mathcal{T}}_{1} \\
& +9 x^{2} m_{N^{*}}^{2} \beta \widehat{\mathcal{T}}_{1}-2 Q^{2} \beta \widehat{\mathcal{T}}_{1}-18 x^{2} m_{N^{*}}^{2}(1+\beta) \widehat{\mathcal{T}}_{3} \\
& +m_{b}\left[x m _ { N ^ { * } } \left(3 \widehat{\mathcal{A}}_{3}-\widehat{\mathcal{V}}_{1}+5 \widehat{\mathcal{V}}_{3}-3 \widehat{\mathcal{P}}_{1}\right.\right. \\
& -\widehat{\mathcal{S}}_{1}+\widehat{\mathcal{A}}_{1}(1+\beta)-7 \widehat{\mathcal{T}}_{1}+\beta\left(5 \widehat{\mathcal{A}}_{3}-\widehat{\mathcal{V}}_{1}+3 \widehat{\mathcal{V}}_{3}\right. \\
& \left.\left.+3 \widehat{\mathcal{P}}_{1}+\widehat{\mathcal{S}}_{1}+7 \widehat{\mathcal{T}}_{1}-12 \widehat{\mathcal{T}}_{3}\right)+12 \widehat{\mathcal{T}}_{3}\right) \\
& \left.\left.-(-1+\beta)\left(\widehat{\mathcal{A}}_{1}+\widehat{\mathcal{V}}_{1}+2 \widehat{\mathcal{T}}_{1}\right) m_{b}\right]\right\} \\
& -\frac{m_{N^{*}}^{2}}{4 x}(-1+\beta)\left(\widehat{\mathcal{A}}_{1}^{M}+\widehat{V}_{1}^{M}+2 \widehat{\mathcal{T}}_{1}^{M}\right) .
\end{aligned}
$$

\section{Invariant function $\Pi_{4}^{I}(p, q)$ for the structure $\gamma_{\mu} q \gamma_{5}$}

$$
\begin{aligned}
& \mathcal{D}_{43}^{I}(x)=\frac{m_{N^{*}}^{2}}{4 x} m_{b}\left\{-3\left(x^{2} m_{N^{*}}^{2}+Q^{2}\right)(-1+\beta) \widetilde{\widetilde{\mathcal{T}}}_{6}\right. \\
& +2 x m_{N^{*}}\left[\widetilde{\mathcal{A}}_{6}(-1+\beta)\right. \\
& +3\left(\widetilde{\widetilde{V}}_{6}(-1+\beta)+\widetilde{\widetilde{\mathcal{T}}}_{6}+t \widetilde{\widetilde{\mathcal{T}}}_{6}\right) \\
& \left.\left.-(11+7 \beta) \widetilde{\widetilde{\mathcal{T}}}_{8}\right] m_{b}-3(-1+\beta) \widetilde{\widetilde{\mathcal{T}}}_{6} m_{b}^{2}\right\} \\
& -\frac{1}{2} m_{N^{*}}^{3}\left\{\widehat{V}_{1}^{M}+3 \widehat{A}_{1}^{M}(-1+\beta)\right. \\
& \left.-\widehat{V}_{1}^{M} \beta+(7+11 \beta) \widehat{T}_{1}^{M}\right\} m_{b}^{2} \text {. } \\
& \mathcal{D}_{42}^{I}(x)=\frac{m_{N^{*}}^{2}}{4 x}\left\{x m _ { N ^ { * } } \left[\widetilde{\widetilde{\mathcal{A}}}_{6}(-1+\beta)\right.\right. \\
& +3\left(\widetilde{\widetilde{V}}_{6}(-1+\beta)+\widetilde{\widetilde{T}}_{6}+\beta \widetilde{\widetilde{\mathcal{T}}}_{6}\right) \\
& \left.\left.-(11+7 \beta) \widetilde{\widetilde{\mathcal{T}}}_{8}\right]+3(-1+\beta) \widetilde{\widetilde{\mathcal{T}}}_{6} m_{b}\right\} \\
& +\frac{m_{N^{*}}}{8 x}\left\{-\left[( x ^ { 2 } m _ { N ^ { * } } ^ { 2 } + Q ^ { 2 } ) \left(4 \tilde{\mathcal{A}}_{2}(-1+\beta)\right.\right.\right. \\
& \left.\left.+4 \widetilde{\mathcal{V}}_{2}(-1+\beta)-3(1+\beta)\left(\widetilde{\mathcal{T}}_{2}+5 \widetilde{\mathcal{T}}_{4}\right)\right)\right] \\
& +x m_{N^{*}}\left[5 \widetilde{\mathcal{A}}_{5}+2 \widetilde{\mathcal{V}}_{2}+\widetilde{\mathcal{V}}_{4}-9 \tilde{\mathcal{V}}_{5}+6 \widetilde{\mathcal{P}}_{2}-2 \widetilde{\mathcal{S}}_{2}\right. \\
& +2 \widetilde{\mathcal{A}}_{2}(1+\beta)-\widetilde{\mathcal{A}}_{4}(1+\beta)+6 \widetilde{\mathcal{T}}_{2} \\
& +8 \widetilde{\mathcal{T}}_{4}+14 \widetilde{\mathcal{T}}_{5}+24 \widetilde{\mathcal{T}}_{7}+\beta\left(9 \tilde{\mathcal{A}}_{5}+2 \widetilde{\mathcal{V}}_{2}+\widetilde{\mathcal{V}}_{4}\right. \\
& -5 \widetilde{\mathcal{V}}_{5}-2\left[3 \widetilde{\mathcal{P}}_{2}-\widetilde{\mathcal{S}}_{2}+3 \widetilde{\mathcal{T}}_{2}+4 \widetilde{\mathcal{T}}_{4}\right. \\
& \left.\left.\left.+7 \tilde{\mathcal{T}}_{5}+12 \tilde{\mathcal{T}}_{7}\right]\right)\right] m_{b}+\left(-4 \tilde{\mathcal{A}}_{2}(-1+\beta)\right. \\
& \left.\left.-4 \widetilde{\mathcal{V}}_{2}(-1+\beta)+3(1+\beta)\left(\widetilde{\mathcal{T}}_{2}+5 \widetilde{\mathcal{T}}_{4}\right)\right) m_{b}^{2}\right\} \\
& -\frac{m_{N^{*}}^{3}}{4}\left[\widehat{V}_{1}^{M}+3 \widehat{A}_{1}^{M}(-1+\beta)\right. \\
& \left.-\widehat{V}_{1}^{M} \beta+(7+11 \beta) \widehat{T}_{1}^{M}\right] \text {. }
\end{aligned}
$$

$$
\begin{aligned}
\mathcal{D}_{41}^{I}(x)= & \frac{m_{N^{*}}}{8 x}\left\{4 \widetilde{\mathcal{A}}_{2}(-1+\beta)+4 \widetilde{\mathcal{V}}_{2}(-1+\beta)\right. \\
& \left.-3(1+\beta)\left(\widetilde{\mathcal{T}}_{2}+5 \widetilde{\mathcal{T}}_{4}\right)\right\} \\
& +\frac{m_{N^{*}}}{4}\left\{\widehat{\mathcal{V}}_{1}-4 \widehat{\mathcal{V}}_{3}+3 \widehat{\mathcal{P}}_{1}+3 \widehat{\mathcal{S}}_{1}\right. \\
& +3 \widehat{\mathcal{A}}_{1}(-1+\beta)+4 \widehat{\mathcal{A}}_{3}(-1+\beta) \\
& -\widehat{\mathcal{V}}_{1} \beta+4 \widehat{\mathcal{V}}_{3} \beta+3 \widehat{\mathcal{P}}_{1} \beta \\
& \left.+3 \widehat{\mathcal{S}}_{1} \beta+7 \widehat{\mathcal{T}}_{1}+11 \beta \widehat{\mathcal{T}}_{1}-18(1+\beta) \widehat{\mathcal{T}}_{3}\right\} .
\end{aligned}
$$

Invariant function $\Pi_{5}^{I}(p, q)$ for the structure $q_{\mu} \gamma_{5}$

$$
\begin{aligned}
& \mathcal{D}_{53}^{I}(x)=\frac{m_{N^{*}}^{2}}{x}\left\{x m_{N^{*}}\left(x^{2} m_{N^{*}}^{2}+Q^{2}\right)(2+\beta) \widetilde{\widetilde{\mathcal{T}}}_{6}\right. \\
& +m_{b}\left[\widetilde{\widetilde{\mathcal{A}}}_{6} x^{2} m_{N^{*}}^{2}(1+3 \beta)+Q^{2}(-1+\beta) \widetilde{\widetilde{\mathcal{T}}}_{6}\right. \\
& +x^{2} m_{N^{*}}^{2}\left(\widetilde{\widetilde{V}}_{6}(3+\beta)+2(-1+\beta) \widetilde{\widetilde{\mathcal{T}}}_{8}\right) \\
& +m_{b}\left(x m _ { N ^ { * } } \left[\widetilde{\widetilde{\mathcal{A}}}_{6}+3 \widetilde{\widetilde{\mathcal{V}}}_{6}-\widetilde{\widetilde{\mathcal{A}}}_{6} \beta-3 \widetilde{\widetilde{\mathcal{V}}}_{6} \beta-\widetilde{\widetilde{\mathcal{T}}}_{6}\right.\right. \\
& \left.\left.\left.\left.-2 \beta \widetilde{\widetilde{\mathcal{T}}}_{6}+10 \widetilde{\widetilde{\mathcal{T}}}_{8}+8 \beta \widetilde{\widetilde{\mathcal{T}}}_{8}\right]+(-1+\beta) \widetilde{\widetilde{\mathcal{T}}}_{6} m_{b}\right)\right]\right\} \\
& +2 m_{N^{*}}^{3}\left\{\widehat{V}_{1}^{M}+\widehat{A}_{1}^{M}(-1+\beta)\right. \\
& \left.-\widehat{V}_{1}^{M} \beta+(4+5 \beta) \widehat{T}_{1}^{M}\right\} m_{b}^{2} \text {. } \\
& \mathcal{D}_{52}^{I}(x)=-\frac{m_{N^{*}}^{2}}{2 x}\left\{x m _ { N ^ { * } } \left[\widetilde{\widetilde{\mathcal{A}}}_{6}(-1+\beta)+3 \widetilde{\widetilde{\mathcal{V}}}_{6}(-1+\beta)\right.\right. \\
& \left.\left.+(5+4 \beta)\left(\widetilde{\widetilde{\mathcal{T}}}_{6}-2 \widetilde{\widetilde{\mathcal{T}}}_{8}\right)\right]+2(-1+\beta) \widetilde{\widetilde{\mathcal{T}}}_{6} m_{b}\right\} \\
& +\frac{m_{N^{*}}}{4 x}\left\{3 x^{2} m_{N^{*}}^{2} \tilde{\mathcal{V}}_{4}-7 x^{2} m_{N^{*}}^{2} \tilde{\mathcal{V}}_{5}\right. \\
& +4 x^{2} m_{N^{*}}^{2} \widetilde{\mathcal{P}}_{2}-4\left(\tilde{\mathcal{A}}_{2}+\widetilde{\mathcal{V}}_{2}\right) Q^{2}-8 x^{2} m_{N^{*}}^{2} \widetilde{\mathcal{S}}_{2} \\
& +\widetilde{\mathcal{A}}_{4} x^{2} m_{N^{*}}^{2}(-1+\beta)-5 \widetilde{\mathcal{A}}_{5} x^{2} m_{N^{*}}^{2}(-1+\beta) \\
& -3 x^{2} m_{N^{*}}^{2} \widetilde{\mathcal{V}}_{4} \beta+7 x^{2} m_{N^{*}}^{2} \widetilde{\mathcal{V}}_{5} \beta \\
& +8 x^{2} m_{N^{*}}^{2} \widetilde{\mathcal{P}}_{2} \beta+4 \widetilde{\mathcal{A}}_{2} Q^{2} \beta+4 \widetilde{\mathcal{V}}_{2} Q^{2} \beta \\
& -4 x^{2} m_{N^{*}}^{2} \widetilde{\mathcal{S}}_{2} \beta \\
& +4 x^{2} m_{N^{*}}^{2} \widetilde{\mathcal{T}}_{2}-2 Q^{2} \widetilde{\mathcal{T}}_{2} \\
& +2 x^{2} m_{N^{*}}^{2} \beta \widetilde{\mathcal{T}}_{2}-4 Q^{2} \beta \widetilde{\mathcal{T}}_{2}-4 x^{2} m_{N^{*}}^{2} \widetilde{\mathcal{T}}_{4} \\
& -14 Q^{2} \widetilde{\mathcal{T}}_{4}-2 x^{2} m_{N^{*}}^{2} \beta \widetilde{\mathcal{T}}_{4}-16 Q^{2} \beta \widetilde{\mathcal{T}}_{4} \\
& +20 x^{2} m_{N^{*}}^{2} \widetilde{\mathcal{T}}_{5}+16 x^{2} m_{N^{*}}^{2} \beta \widetilde{\mathcal{T}}_{5} \\
& +36 x^{2} m_{N^{*}}^{2}(1+\beta) \widetilde{\mathcal{T}}_{7}+m_{b}\left[-\left(x m _ { N ^ { * } } \left[\tilde{\mathcal{A}}_{4}+\widetilde{\mathcal{A}}_{5}\right.\right.\right. \\
& -3 \widetilde{\mathcal{V}}_{4}-3 \widetilde{\mathcal{V}}_{5}+3 \widetilde{\mathcal{A}}_{4} \beta+3 \tilde{\mathcal{A}}_{5} \beta \\
& +2 \widetilde{\mathcal{T}}_{2}-2 \widetilde{\mathcal{T}}_{4}+4 \widetilde{\mathcal{T}}_{5}-\beta\left(\tilde{\mathcal{V}}_{4}+\tilde{\mathcal{V}}_{5}\right. \\
& \left.\left.\left.+2 \widetilde{\mathcal{T}}_{2}-2 \widetilde{\mathcal{T}}_{4}+4 \widetilde{\mathcal{T}}_{5}\right)\right]\right) \\
& -2\left(2 \tilde{\mathcal{V}}_{2}+\widetilde{\mathcal{T}}_{2}+7 \tilde{\mathcal{T}}_{4}+2\left[\tilde{\mathcal{A}}_{2}-\tilde{\mathcal{A}}_{2} \beta\right.\right. \\
& \left.\left.\left.\left.+\beta\left(-\widetilde{\mathcal{V}}_{2}+\widetilde{\mathcal{T}}_{2}+4 \widetilde{\mathcal{T}}_{4}\right)\right]\right) m_{b}\right]\right\} \\
& +m_{N^{*}}^{3}\left\{\widehat{V}_{1}^{M}+\widehat{A}_{1}^{M}(-1+\beta)-\widehat{V}_{1}^{M} \beta+(4+5 \beta) \widehat{T}_{1}^{M}\right\} .
\end{aligned}
$$




$$
\begin{aligned}
\mathcal{D}_{51}^{I}(x)= & \frac{m_{N^{*}}}{2 x}\left\{2 \widetilde{\mathcal{V}}_{2}+\widetilde{\mathcal{T}}_{2}+7 \widetilde{\mathcal{T}}_{4}\right. \\
& \left.+2\left[\widetilde{\mathcal{A}}_{2}-\widetilde{\mathcal{A}}_{2} \beta+\beta\left(-\widetilde{\mathcal{V}}_{2}+\widetilde{\mathcal{T}}_{2}+4 \widetilde{\mathcal{T}}_{4}\right)\right]\right\} \\
& +\frac{1}{2}\left\{-\left[m _ { N ^ { * } } \left(2 \widehat{\mathcal{V}}_{1}-5 \widehat{\mathcal{V}}_{3}\right.\right.\right. \\
& +2 \widehat{\mathcal{P}}_{1}+4 \widehat{\mathcal{S}}_{1}+2 \widehat{\mathcal{A}}_{1}(-1+\beta)+3 \widehat{\mathcal{A}}_{3}(-1+\beta) \\
& +8 \widehat{\mathcal{T}}_{1}+\beta\left[-2 \widehat{\mathcal{V}}_{1}+5 \widehat{\mathcal{V}}_{3}+4 \widehat{\mathcal{P}}_{1}\right. \\
& \left.\left.\left.\left.+2 \widehat{\mathcal{S}}_{1}+10 \widehat{\mathcal{T}}_{1}\right]\right)\right]+18 m_{N^{*}}(1+\beta) \widehat{\mathcal{T}}_{3}\right\} .
\end{aligned}
$$

Invariant function $\Pi_{6}^{I}(p, q)$ for the structure $q_{\mu} q \gamma_{5}$

$$
\begin{aligned}
\mathcal{D}_{63}^{I}(x)= & \frac{m_{N^{*}}^{2}}{x}\left\{\left(x^{2} m_{N^{*}}^{2}+Q^{2}\right)(2+\beta) \widetilde{\widetilde{\mathcal{T}}}_{6}\right. \\
& +x m_{N^{*}}\left(\widetilde{\widetilde{\mathcal{A}}}_{6}+3 \widetilde{\widetilde{\mathcal{A}}}_{6} \beta+\widetilde{\widetilde{\mathcal{V}}}_{6}(3+\beta)\right. \\
& \left.\left.-(-1+\beta)\left(\widetilde{\widetilde{\mathcal{T}}}_{6}-2 \widetilde{\widetilde{\mathcal{T}}}_{8}\right)\right) m_{b}+(2+\beta) \widetilde{\widetilde{\mathcal{T}}}_{6} m_{b}^{2}\right\} . \\
\mathcal{D}_{62}^{I}(x)= & -\frac{m_{N^{*}}^{2}}{x}(2+\beta) \widetilde{\widetilde{\mathcal{T}}}_{6}+\frac{m_{N^{*}}^{2}}{x}\left\{-\widetilde{\mathcal{A}}_{4}+5 \widetilde{\mathcal{A}}_{5}+4 \widetilde{\mathcal{V}}_{2}\right. \\
& +3 \widetilde{\mathcal{V}}_{4}-7 \widetilde{\mathcal{V}}_{5}+4 \widetilde{\mathcal{P}}_{2}-8 \widetilde{\mathcal{S}}_{2} \\
& -4 \widetilde{\mathcal{A}}_{2}(-1+\beta)+6 \widetilde{\mathcal{T}}_{2}+10 \widetilde{\mathcal{T}}_{4}+20 \widetilde{\mathcal{T}}_{5} \\
& +36 \widetilde{\mathcal{T}}_{7}+\beta\left[\widetilde{\mathcal{A}}_{4}-5 \widetilde{\mathcal{A}}_{5}-4 \widetilde{\mathcal{V}}_{2}-3 \widetilde{\mathcal{V}}_{4}\right. \\
& +7 \widetilde{\mathcal{V}}_{5}+8 \widetilde{\mathcal{P}}_{2}-4 \widetilde{\mathcal{S}}_{2} \\
& \left.\left.+6 \widetilde{\mathcal{T}}_{2}+14 \widetilde{\mathcal{T}}_{4}+16 \widetilde{\mathcal{T}}_{5}+36 \widetilde{\mathcal{T}}_{7}\right]\right\} . \\
\mathcal{D}_{61}^{I}(x)= & 0 .
\end{aligned}
$$

\section{Invariant functions of the transition current $\left(\bar{b} i \sigma_{\mu \nu} q^{v} d\right)$}

In this section we present the expressions for the invariant functions $\Pi_{1}^{I I}, \Pi_{2}^{I I}, \Pi_{3}^{I I}$, and $\Pi_{4}^{I I}$ for coefficients of the structures $p_{\mu} q \gamma_{5}, q_{\mu} q \gamma_{5}, q_{\mu} \gamma_{5}$, and $\gamma_{\mu} \gamma_{5}$, respectively, for the transition current $\left(\bar{b} i \sigma_{\mu \nu} q^{\nu} d\right)$ which appear in the sum rules.

Invariant function $\Pi_{1}^{I I}(p, q)$ for the structure $p_{\mu} q \gamma_{5}$

$$
\begin{aligned}
\mathcal{D}_{13}^{I I}(x)= & m_{N^{*}}^{2}\left\{x m_{N^{*}}\left(x^{2} m_{N^{*}}^{2}+Q^{2}\right)(-1+\beta) \widetilde{\widetilde{\mathcal{T}}}_{6}\right. \\
& +m_{b}\left[-\left(\widetilde{\widetilde{\mathcal{A}}}_{6} x^{2} m_{N^{*}}^{2}(-1+\beta)\right)\right. \\
& +Q^{2}(3+\beta) \widetilde{\widetilde{\mathcal{T}}}_{6}+x^{2} m_{N^{*}}^{2}(-1+\beta)\left(\widetilde{\widetilde{\mathcal{V}}}_{6}-2 \widetilde{\widetilde{\mathcal{T}}}_{8}\right) \\
& -x m_{N^{*}}\left(3 \widetilde{\widetilde{\mathcal{A}}}_{6}(1+\beta)+3 \widetilde{\widetilde{V}}_{6}(1+\beta)\right. \\
& \left.\left.\left.+(-1+\beta)\left(\widetilde{\widetilde{\mathcal{T}}}_{6}-6 \widetilde{\widetilde{\mathcal{T}}}_{8}\right)\right) m_{b}\right]\right\} \\
& +m_{N^{*}}^{2} m_{b}^{2}\left\{3 x m _ { N ^ { * } } \left(\left(\widehat{A}_{1}^{M}-\widehat{V}_{1}^{M}\right)(1+\beta)\right.\right. \\
& \left.+2(-1+\beta) \widehat{T}_{1}^{M}\right) \\
& \left.+(-1+\beta)\left(\widehat{A}_{1}^{M}+\widehat{V}_{1}^{M}-2 \widehat{T}_{1}^{M}\right) m_{b}\right\} .
\end{aligned}
$$

$$
\begin{aligned}
& \mathcal{D}_{12}^{I I}(x)=\frac{m_{N^{*}}^{2}}{2}\left\{-\left[x m _ { N ^ { * } } \left(3 \widetilde{\widetilde{\mathcal{A}}}_{6}(1+\beta)+3 \widetilde{\widetilde{\mathcal{V}}}_{6}(1+\beta)\right.\right.\right. \\
& \left.\left.+2(-1+\beta)\left(\widetilde{\widetilde{\mathcal{T}}}_{6}-3 \widetilde{\widetilde{\mathcal{T}}}_{8}\right)\right)\right] \\
& \left.+(-1+\beta) \widetilde{\widetilde{\mathcal{T}}}_{6} m_{b}\right\}+\frac{m_{N^{*}}}{4}\left\{-3 x^{2} m_{N^{*}}^{2} \widetilde{\mathcal{V}}_{4}\right. \\
& +9 x^{2} m_{N^{*}}^{2} \widetilde{\mathcal{V}}_{5}-4 x^{2} m_{N^{*}}^{2} \widetilde{\mathcal{P}}_{2}+4 \widetilde{\mathcal{A}}_{2} Q^{2} \\
& +8 \widetilde{\mathcal{V}}_{2} Q^{2}+4 x^{2} m_{N^{*}}^{2} \widetilde{\mathcal{S}}_{2}-3 x^{2} m_{N^{*}}^{2} \widetilde{\mathcal{V}}_{4} \beta \\
& +9 x^{2} m_{N^{*}}^{2} \tilde{\mathcal{V}}_{5} \beta+4 x^{2} m_{N^{*}}^{2} \widetilde{\mathcal{P}}_{2} \beta \\
& +8 \widetilde{\mathcal{A}}_{2} Q^{2} \beta+4 \widetilde{\mathcal{V}}_{2} Q^{2} \beta \\
& -4 x^{2} m_{N^{*}}^{2} \widetilde{\mathcal{S}}_{2} \beta+3 \widetilde{\mathcal{A}}_{4} x^{2} m_{N^{*}}^{2}(1+\beta) \\
& -9 \widetilde{\mathcal{A}}_{5} x^{2} m_{N^{*}}^{2}(1+\beta)-2 x^{2} m_{N^{*}}^{2} \widetilde{\mathcal{T}}_{2}+4 Q^{2} \widetilde{\mathcal{T}}_{2} \\
& +2 x^{2} m_{N^{*}}^{2} \beta \widetilde{\mathcal{T}}_{2}-4 Q^{2} \beta \widetilde{\mathcal{T}}_{2}+2 x^{2} m_{N^{*}}^{2} \widetilde{\mathcal{T}}_{4} \\
& +12 Q^{2} \widetilde{\mathcal{T}}_{4}-2 x^{2} m_{N^{*}}^{2} \beta \widetilde{\mathcal{T}}_{4}-12 Q^{2} \beta \widetilde{\mathcal{T}}_{4} \\
& -12 x^{2} m_{N^{*}}^{2} \widetilde{\mathcal{T}}_{5}+12 x^{2} m_{N^{*}}^{2} \beta \widetilde{\mathcal{T}}_{5} \\
& +24 x^{2} m_{N^{*}}^{2}(-1+\beta) \widetilde{\mathcal{T}}_{7} \\
& +m_{b}\left[x m _ { N ^ { * } } ( - 1 + \beta ) \left(2 \widetilde{\mathcal{A}}_{2}+\widetilde{\mathcal{A}}_{4}+\widetilde{\mathcal{A}}_{5}\right.\right. \\
& \left.-2 \widetilde{\mathcal{V}}_{2}+\widetilde{\mathcal{V}}_{4}+\widetilde{\mathcal{V}}_{5}+4 \widetilde{\mathcal{T}}_{4}-4 \widetilde{\mathcal{T}}_{5}\right) \\
& +2\left(3 \widetilde{\mathcal{A}}_{2}(1+\beta)+3 \widetilde{\mathcal{V}}_{2}(1+\beta)\right. \\
& \left.\left.\left.-(-1+\beta)\left(\widetilde{\mathcal{T}}_{2}+5 \widetilde{\mathcal{T}}_{4}\right)\right) m_{b}\right]\right\} \\
& +\frac{3 x m_{N^{*}}^{3}}{2}\left\{\left(\widehat{A}_{1}^{M}-\widehat{V}_{1}^{M}\right)(1+\beta)\right. \\
& \left.+2(-1+\beta) \widehat{T}_{1}^{M}\right\} \text {. } \\
& \mathcal{D}_{11}^{I I}(x)=-\frac{x m_{N^{*}}}{2}\left\{6 \widehat{\mathcal{A}}_{3}-3 \widehat{\mathcal{V}}_{1}+6 \widehat{\mathcal{V}}_{3}+3 \widehat{\mathcal{A}}_{1}(1+\beta)\right. \\
& +\beta\left[6 \widehat{\mathcal{A}}_{3}-3 \widehat{\mathcal{V}}_{1}+2\left(3 \widehat{\mathcal{V}}_{3}\right.\right. \\
& \left.\left.+\widehat{\mathcal{P}}_{1}+\widehat{\mathcal{S}}_{1}+3 \widehat{\mathcal{T}}_{1}-6 \widehat{\mathcal{T}}_{3}\right)\right] \\
& \left.-2\left(\widehat{\mathcal{P}}_{1}+\widehat{\mathcal{S}}_{1}+3 \widehat{\mathcal{T}}_{1}-6 \widehat{\mathcal{T}}_{3}\right)\right\} \\
& \left.-(-1+\beta)\left[\widehat{\mathcal{A}}_{1}+\widehat{\mathcal{V}}_{1}-2 \widehat{\mathcal{T}}_{1}\right) m_{b}\right] \text {. }
\end{aligned}
$$

Invariant function $\Pi_{2}^{I I}(p, q)$ for the structure $q_{\mu} q \gamma_{5}$

$$
\begin{aligned}
\mathcal{D}_{23}^{I I}(x)= & \frac{m_{N^{*}}^{2}}{4 x}\left\{-4 x m_{N^{*}}\left(x^{2} m_{N^{*}}^{2}+Q^{2}\right)(-1+\beta) \widetilde{\widetilde{\mathcal{T}}}_{6}\right. \\
& +m_{b}\left[4 \widetilde{\widetilde{\mathcal{A}}}_{6} x^{2} m_{N^{*}}^{2}(-1+\beta)\right. \\
& -Q^{2}(7+3 \beta) \widetilde{\widetilde{\mathcal{T}}}_{6}+x^{2} m_{N^{*}}^{2}\left(-4 \widetilde{\widetilde{\mathcal{V}}}_{6}(-1+\beta)\right. \\
& \left.+(5+\beta) \widetilde{\widetilde{\mathcal{T}}}_{6}+8(-1+\beta) \widetilde{\widetilde{\mathcal{T}}}_{8}\right) \\
& +m_{b}\left(2 x m _ { N ^ { * } } \left[3 \widetilde{\widetilde{\mathcal{A}}}_{6}(1+\beta)+3 \widetilde{\widetilde{\mathcal{V}}}_{6}(1+\beta)\right.\right. \\
& \left.\left.\left.\left.-(-1+\beta)\left(\widetilde{\widetilde{\mathcal{T}}}_{6}+5 \widetilde{\widetilde{\mathcal{T}}}_{8}\right)\right]-(7+3 \beta) \widetilde{\widetilde{\mathcal{T}}}_{6} m_{b}\right)\right]\right\} \\
& +\frac{m_{N^{*}}^{3}}{2}\left\{-3\left(\widehat{A}_{1}^{M}-\widehat{V}_{1}^{M}\right)(1+\beta)-5(-1+\beta) \widehat{T}_{1}^{M}\right\} m_{b}^{2} .
\end{aligned}
$$




$$
\begin{aligned}
& \mathcal{D}_{22}^{I I}(x)=\frac{m_{N^{*}}^{2}}{4 x}\left\{x m _ { N ^ { * } } \left[3\left(\widetilde{\widetilde{\mathcal{A}}}_{6}+\widetilde{\widetilde{\mathcal{V}}}_{6}\right)(1+\beta)+3(-1+\beta) \widetilde{\widetilde{\mathcal{T}}}_{6}\right.\right. \\
& \left.\left.-5(-1+\beta) \widetilde{\widetilde{\mathcal{T}}}_{8}\right]+(7+3 \beta) \widetilde{\widetilde{\mathcal{T}}}_{6} m_{b}\right\} \\
& +\frac{m_{N^{*}}}{8 x}\left\{18 \tilde{\mathcal{A}}_{5} x^{2} m_{N^{*}}^{2}+8 x^{2} m_{N^{*}}^{2} \tilde{\mathcal{V}}_{2}+6 x^{2} m_{N^{*}}^{2} \tilde{\mathcal{V}}_{4}\right. \\
& -18 x^{2} m_{N^{*}}^{2} \widetilde{\mathcal{V}}_{5}+8 x^{2} m_{N^{*}}^{2} \widetilde{\mathcal{P}}_{2}-8 \widetilde{\mathcal{V}}_{2} Q^{2} \\
& -8 x^{2} m_{N^{*}}^{2} \widetilde{\mathcal{S}}_{2}+18 \widetilde{\mathcal{A}}_{5} x^{2} m_{N^{*}}^{2} \beta+4 x^{2} m_{N^{*}}^{2} \widetilde{\mathcal{V}}_{2} \beta \\
& +6 x^{2} m_{N^{*}}^{2} \tilde{\mathcal{V}}_{4} \beta \\
& -18 x^{2} m_{N^{*}}^{2} \widetilde{\mathcal{V}}_{5} \beta-8 x^{2} m_{N^{*}}^{2} \widetilde{\mathcal{P}}_{2} \beta \\
& -4 \widetilde{\mathcal{V}}_{2} Q^{2} \beta+8 x^{2} m_{N^{*}}^{2} \widetilde{\mathcal{S}}_{2} \beta-6 \widetilde{\mathcal{A}}_{4} x^{2} m_{N^{*}}^{2}(1+\beta) \\
& +4 \widetilde{\mathcal{A}}_{2}\left(x^{2} m_{N^{*}}^{2}-Q^{2}\right)(1+2 \beta)+9 x^{2} m_{N^{*}}^{2} \widetilde{\mathcal{I}}_{2} \\
& -3 Q^{2} \widetilde{\mathcal{T}}_{2}-9 x^{2} m_{N^{*}}^{2} \beta \widetilde{\mathcal{T}}_{2}+3 Q^{2} \beta \widetilde{\mathcal{T}}_{2}+9 x^{2} m_{N^{*}}^{2} \widetilde{\mathcal{T}}_{4} \\
& -11 Q^{2} \widetilde{\mathcal{T}}_{4}-9 x^{2} m_{N^{*}}^{2} \beta \widetilde{\mathcal{T}}_{4}+11 Q^{2} \beta \widetilde{\mathcal{T}}_{4} \\
& +24 x^{2} m_{N^{*}}^{2} \widetilde{\mathcal{T}}_{5}-24 x^{2} m_{N^{*}}^{2} \beta \widetilde{\mathcal{T}}_{5} \\
& -48 x^{2} m_{N^{*}}^{2}(-1+\beta) \widetilde{\mathcal{T}}_{7} \\
& +m_{b}\left\{x m _ { N ^ { * } } \left[3 \widetilde{\mathcal{A}}_{4}-5 \widetilde{\mathcal{A}}_{5}-6 \tilde{\mathcal{V}}_{2}\right.\right. \\
& -\widetilde{\mathcal{V}}_{4}+7 \widetilde{\mathcal{V}}_{5}+2 \widetilde{\mathcal{A}}_{2}(-1+\beta) \\
& -2\left(\widetilde{\mathcal{P}}_{2}-\widetilde{\mathcal{S}}_{2}+\widetilde{\mathcal{T}}_{2}+4 \widetilde{\mathcal{T}}_{4}+9 \widetilde{\mathcal{T}}_{5}+14 \widetilde{\mathcal{T}}_{7}\right) \\
& +\beta\left(-3 \tilde{\mathcal{A}}_{4}+5 \tilde{\mathcal{A}}_{5}\right. \\
& +6 \widetilde{\mathcal{V}}_{2}+\widetilde{\mathcal{V}}_{4}-7 \widetilde{\mathcal{V}}_{5}-2\left[\widetilde{\mathcal{P}}_{2}-\widetilde{\mathcal{S}}_{2}\right. \\
& \left.\left.\left.+\widetilde{\mathcal{T}}_{2}+8 \widetilde{\mathcal{T}}_{4}+5 \widetilde{\mathcal{T}}_{5}+14 \widetilde{\mathcal{T}}_{7}\right]\right)\right] \\
& -\left[4 \widetilde{\mathcal{V}}_{2}(2+\beta)+\widetilde{\mathcal{A}}_{2}(4+8 \beta)\right. \\
& \left.\left.\left.-(-1+\beta)\left(3 \widetilde{\mathcal{T}}_{2}+11 \widetilde{\mathcal{T}}_{4}\right)\right] m_{b}\right\}\right\} \\
& +\frac{m_{N^{*}}^{3}}{4}\left\{-3\left(\widehat{A}_{1}^{M}-\widehat{V}_{1}^{M}\right)(1+\beta)\right. \\
& \left.-5(-1+\beta) \widehat{T}_{1}^{M}\right\} \text {. } \\
& \mathcal{D}_{21}^{I I}(x)=\frac{m_{N^{*}}}{8 x}\left\{4 \widetilde{\mathcal{V}}_{2}(2+\beta)+\widetilde{\mathcal{A}}_{2}(4+8 \beta)\right. \\
& \left.-(-1+\beta)\left(3 \widetilde{\mathcal{T}}_{2}+11 \widetilde{\mathcal{T}}_{4}\right)\right\}+\frac{m_{N^{*}}}{4}\left\{5 \widehat{\mathcal{A}}_{3}\right. \\
& -3 \widehat{\mathcal{V}}_{1}+7 \widehat{\mathcal{V}}_{3}-\widehat{\mathcal{P}}_{1}-3 \widehat{\mathcal{S}}_{1}+3 \widehat{\mathcal{A}}_{1}(1+\beta)-5 \widehat{\mathcal{T}}_{1} \\
& +\beta\left[7 \widehat{\mathcal{A}}_{3}-3 \widehat{\mathcal{V}}_{1}+5 \widehat{\mathcal{V}}_{3}+\widehat{\mathcal{P}}_{1}\right. \\
& \left.\left.+3 \widehat{\mathcal{S}}_{1}+5 \widehat{\mathcal{T}}_{1}-12 \widehat{\mathcal{T}}_{3}\right]+12 \widehat{\mathcal{T}}_{3}\right\} \text {. }
\end{aligned}
$$

Invariant function $\Pi_{3}^{I I}(p, q)$ for the structure $q_{\mu} \gamma_{5}$

$$
\begin{aligned}
\mathcal{D}_{33}^{I I}(x)= & \frac{m_{N^{*}}^{2}}{4 x}\left\{4 Q^{2}\left(x^{2} m_{N^{*}}^{2}+Q^{2}\right)(-1+\beta) \widetilde{\widetilde{\mathcal{T}}}_{6}\right. \\
& +m_{b}\left[x m _ { N ^ { * } } \left(-4 \widetilde{\widetilde{\mathcal{A}}}_{6} Q^{2}(-1+\beta)\right.\right. \\
& +4 \widetilde{\widetilde{\mathcal{V}}}_{6} Q^{2}(-1+\beta)+\left(x^{2} m_{N^{*}}^{2}(5+\beta)\right. \\
& \left.\left.-Q^{2}(7+3 \beta)\right) \widetilde{\widetilde{\mathcal{T}}}_{6}-8 Q^{2}(-1+\beta) \widetilde{\widetilde{\mathcal{T}}}_{8}\right) \\
& +m_{b}\left(-6 \widetilde{\widetilde{\mathcal{A}}}_{6} x^{2} m_{N^{*}}^{2}(1+\beta)\right.
\end{aligned}
$$

$$
\begin{aligned}
& +9 Q^{2}(-1+\beta) \widetilde{\widetilde{\mathcal{T}}}_{6}-x^{2} m_{N^{*}}^{2}\left(6 \widetilde{\widetilde{\mathcal{V}}}_{6}(1+\beta)\right. \\
& \left.+(-1+\beta)\left(5 \widetilde{\widetilde{\mathcal{T}}}_{6}-14 \widetilde{\widetilde{\mathcal{T}}}_{8}\right)\right) \\
& +m_{b}\left(x m _ { N ^ { * } } \left(2 \widetilde{\widetilde{\mathcal{A}}}_{6}(-1+\beta)+6 \widetilde{\widetilde{\mathcal{V}}}_{6}(-1+\beta)\right.\right. \\
& \left.-(5+\beta) \widetilde{\widetilde{\mathcal{T}}}_{6}-2(5+9 \beta) \widetilde{\widetilde{\mathcal{T}}}_{8}\right) \\
& \left.\left.\left.\left.+5(-1+\beta) \widetilde{\widetilde{T}}_{6} m_{b}\right)\right)\right]\right\} \\
& +\frac{m_{N^{*}}^{2}}{2 x} m_{b}^{2}\left\{\widehat{A}_{1}^{M}\left(Q^{2}-Q^{2} \beta+2 x^{2} m_{N^{*}}^{2}(1+2 \beta)\right)\right. \\
& -Q^{2}(-1+\beta)\left(\widehat{V}_{1}^{M}+2 \widehat{T}_{1}^{M}\right) \\
& -x^{2} m_{N^{*}}^{2}\left(2 \widehat{V}_{1}^{M}(2+\beta)-9(-1+\beta) \widehat{T}_{1}^{M}\right) \\
& +m_{b}\left[x m _ { N ^ { * } } \left(\widehat{A}_{1}^{M}+3 \widehat{V}_{1}^{M}(-1+\beta)\right.\right. \\
& \left.-\widehat{A}_{1}^{M} \beta-(5+9 \beta) \widehat{T}_{1}^{M}\right) \\
& \left.\left.-(-1+\beta)\left(\widehat{A}_{1}^{M}+\widehat{V}_{1}^{M}+2 \widehat{T}_{1}^{M}\right) m_{b}\right]\right\} . \\
& \mathcal{D}_{32}^{I I}(x)=\frac{m_{N^{*}}^{2}}{8 x}\left\{-6 \widetilde{\widetilde{\mathcal{A}}}_{6} x^{2} m_{N^{*}}^{2}(1+\beta)\right. \\
& -7 Q^{2}(-1+\beta) \widetilde{\widetilde{\mathcal{T}}}_{6}-x^{2} m_{N^{*}}^{2}\left(6 \widetilde{\widetilde{V}}_{6}(1+\beta)\right. \\
& \left.+(-1+\beta)\left(9 \widetilde{\widetilde{T}}_{6}-14 \widetilde{\widetilde{\mathcal{T}}}_{8}\right)\right) \\
& +m_{b}\left[x m _ { N ^ { * } } \left(4 \widetilde{\widetilde{V}}_{6}+4 \widetilde{\widetilde{\mathcal{A}}}_{6}(-1+\beta)-4 \widetilde{\widetilde{V}}_{6} \beta\right.\right. \\
& \left.+15 \widetilde{\widetilde{T}}_{6}+11 \beta \widetilde{\widetilde{\mathcal{T}}}_{6}+8(-1+\beta) \widetilde{\widetilde{\mathcal{T}}}_{8}\right) \\
& \left.\left.-9(-1+\beta) \widetilde{\widetilde{T}}_{6} m_{b}\right]\right\} \\
& +\frac{m_{N^{*}}}{8 x}\left\{x m _ { N ^ { * } } \left[4 \widetilde{\mathcal{A}}_{2}\left(x^{2} m_{N^{*}}^{2}-Q^{2}\right)(1+2 \beta)\right.\right. \\
& +x^{2} m_{N^{*}}^{2}\left[4 \widetilde{\mathcal{V}}_{2}(2+\beta)-(-1+\beta)\left(5 \widetilde{\mathcal{T}}_{2}+13 \widetilde{\mathcal{T}}_{4}\right)\right] \\
& +Q^{2}\left(-8 \widetilde{\mathcal{V}}_{2}-6 \widetilde{\mathcal{V}}_{4}+18 \tilde{\mathcal{V}}_{5}-8 \widetilde{\mathcal{P}}_{2}+8 \widetilde{\mathcal{S}}_{2}\right. \\
& +6 \widetilde{\mathcal{A}}_{4}(1+\beta)-18 \tilde{\mathcal{A}}_{5}(1+\beta)-7 \widetilde{\mathcal{T}}_{2}-7 \widetilde{\mathcal{T}}_{4} \\
& -24\left(\widetilde{\mathcal{T}}_{5}+2 \widetilde{\mathcal{T}}_{7}\right)+t\left[-4 \tilde{\mathcal{V}}_{2}-6 \tilde{\mathcal{V}}_{4}+18 \tilde{\mathcal{V}}_{5}\right. \\
& \left.\left.\left.+8 \widetilde{\mathcal{P}}_{2}-8 \widetilde{\mathcal{S}}_{2}+7 \widetilde{\mathcal{T}}_{2}+7 \widetilde{\mathcal{T}}_{4}+24 \widetilde{\mathcal{T}}_{5}+48 \widetilde{\mathcal{T}}_{7}\right]\right)\right] \\
& -\left[3 x^{2} m_{N^{*}}^{2} \widetilde{\mathcal{V}}_{4}-5 x^{2} m_{N^{*}}^{2} \widetilde{\mathcal{V}}_{5}+2 x^{2} m_{N^{*}}^{2} \widetilde{\mathcal{P}}_{2}\right. \\
& -2\left(\tilde{\mathcal{A}}_{2}+3 \tilde{\mathcal{V}}_{2}\right) Q^{2}-2 x^{2} m_{N^{*}}^{2} \widetilde{\mathcal{S}}_{2} \\
& +\widetilde{\mathcal{A}}_{4} x^{2} m_{N^{*}}^{2}(-1+\beta)-7 \widetilde{\mathcal{A}}_{5} x^{2} m_{N^{*}}^{2}(-1+\beta) \\
& -3 x^{2} m_{N^{*}}^{2} \tilde{\mathcal{V}}_{4} \beta \\
& +5 x^{2} m_{N^{*}}^{2} \widetilde{\mathcal{V}}_{5} \beta+2 x^{2} m_{N^{*}}^{2} \widetilde{\mathcal{P}}_{2} \beta \\
& +2 \widetilde{\mathcal{A}}_{2} Q^{2} \beta+6 \widetilde{\mathcal{V}}_{2} Q^{2} \beta-2 x^{2} m_{N^{*}}^{2} \widetilde{\mathcal{S}}_{2} \beta \\
& -5 x^{2} m_{N^{*}}^{2} \widetilde{\mathcal{T}}_{2}+5 Q^{2} \widetilde{\mathcal{T}}_{2}-x^{2} m_{N^{*}}^{2} \beta \widetilde{\mathcal{T}}_{2}+Q^{2} \beta \widetilde{\mathcal{T}}_{2} \\
& +5 x^{2} m_{N^{*}}^{2} \widetilde{\mathcal{T}}_{4}-15 Q^{2} \widetilde{\mathcal{T}}_{4}+x^{2} m_{N^{*}}^{2} \beta \widetilde{\mathcal{T}}_{4} \\
& -19 Q^{2} \beta \widetilde{\mathcal{T}}_{4}+10 x^{2} m_{N^{*}}^{2} \widetilde{\mathcal{T}}_{5}+18 x^{2} m_{N^{*}}^{2} \beta \widetilde{\mathcal{T}}_{5} \\
& \left.+28 x^{2} m_{N^{*}}^{2}(1+\beta) \tilde{\mathcal{T}}_{7}\right] m_{b} \\
& +x m_{N^{*}}\left[-11 \widetilde{\mathcal{A}}_{5}-2 \widetilde{\mathcal{V}}_{2}-3 \widetilde{\mathcal{V}}_{4}+7 \widetilde{\mathcal{V}}_{5}-6 \widetilde{\mathcal{P}}_{2}+2 \widetilde{\mathcal{S}}_{2}\right. \\
& -2 \widetilde{\mathcal{A}}_{2}(-1+\beta)+3 \widetilde{\mathcal{A}}_{4}(1+\beta)-5 \widetilde{\mathcal{T}}_{2}+\widetilde{\mathcal{T}}_{4}-14 \widetilde{\mathcal{T}}_{5}
\end{aligned}
$$




$$
\begin{aligned}
& -24 \widetilde{\mathcal{T}}_{7}+\beta\left(-7 \widetilde{\mathcal{A}}_{5}+2 \widetilde{\mathcal{V}}_{2}-3 \tilde{\mathcal{V}}_{4}+11 \tilde{\mathcal{V}}_{5}+6 \widetilde{\mathcal{P}}_{2}\right. \\
& \left.\left.-2 \widetilde{\mathcal{S}}_{2}+5 \widetilde{\mathcal{T}}_{2}-\widetilde{\mathcal{T}}_{4}+14 \widetilde{\mathcal{T}}_{5}+24 \widetilde{\mathcal{T}}_{7}\right)\right] m_{b}^{2} \\
& +\left[-2 \tilde{\mathcal{A}}_{2}(-1+\beta)-6 \tilde{\mathcal{V}}_{2}(-1+\beta)\right. \\
& \left.\left.-(5+\beta) \widetilde{\mathcal{T}}_{2}+(15+19 \beta) \widetilde{\mathcal{T}}_{4}\right] m_{b}^{3}\right\} \\
& +\frac{m_{N^{*}}^{2}}{4 x}\left\{\widehat{A}_{1}^{M}\left[Q^{2}-Q^{2} \beta+2 x^{2} m_{N^{*}}^{2}(1+2 \beta)\right]\right. \\
& -Q^{2}(-1+\beta)\left(\widehat{V}_{1}^{M}+2 \widehat{T}_{1}^{M}\right) \\
& -x^{2} m_{N^{*}}^{2}\left[2 \widehat{V}_{1}^{M}(2+\beta)-9(-1+\beta) \widehat{T}_{1}^{M}\right] \\
& \left.+(-1+\beta)\left(\widehat{A}_{1}^{M}+\widehat{V}_{1}^{M}+2 \widehat{T}_{1}^{M}\right) m_{b}^{2}\right\} \text {. } \\
& \mathcal{D}_{31}^{I I}(x)=-\frac{m_{N^{*}}^{2}}{8 x}(-1+\beta) \widetilde{\widetilde{\mathcal{T}}}_{6}+\frac{m_{N^{*}}}{8 x} \\
& \left\{x m _ { N ^ { * } } \left[7 \tilde{\mathcal{A}}_{5}+10 \tilde{\mathcal{V}}_{2}+3 \tilde{\mathcal{V}}_{4}-11 \tilde{\mathcal{V}}_{5}-3 \widetilde{\mathcal{A}}_{4}(1+\beta)\right.\right. \\
& +2 \widetilde{\mathcal{A}}_{2}(1+5 \beta)+2\left(\widetilde{\mathcal{P}}_{2}-3 \widetilde{\mathcal{S}}_{2}+3 \widetilde{\mathcal{T}}_{2}+6 \widetilde{\mathcal{T}}_{4}\right. \\
& \left.+5 \widetilde{\mathcal{T}}_{5}+12 \widetilde{\mathcal{T}}_{7}\right)+\beta\left(11 \tilde{\mathcal{A}}_{5}+2 \tilde{\mathcal{V}}_{2}+3 \tilde{\mathcal{V}}_{4}\right. \\
& -7 \widetilde{\mathcal{V}}_{5}-2\left[\widetilde{\mathcal{P}}_{2}-3 \widetilde{\mathcal{S}}_{2}+3 \widetilde{\mathcal{T}}_{2}\right. \\
& \left.\left.\left.+6 \widetilde{\mathcal{T}}_{4}+5 \widetilde{\mathcal{T}}_{5}+12 \widetilde{\mathcal{T}}_{7}\right]\right)\right] \\
& +\left[2 \widetilde{\mathcal{A}}_{2}(-1+\beta)+6 \widetilde{\mathcal{V}}_{2}(-1+\beta)\right. \\
& \left.\left.+(5+\beta) \widetilde{\mathcal{T}}_{2}-(15+19 \beta) \widetilde{\mathcal{T}}_{4}\right] m_{b}\right\} \\
& +\frac{1}{4 x}\left\{4 x^{2} m_{N^{*}}^{2} \widehat{\mathcal{V}}_{1}-5 x^{2} m_{N^{*}}^{2} \widehat{\mathcal{V}}_{3}\right. \\
& +3 x^{2} m_{N^{*}}^{2} \widehat{\mathcal{P}}_{1}-\widehat{\mathcal{V}}_{1} Q^{2}+x^{2} m_{N^{*}}^{2} \widehat{\mathcal{S}}_{1} \\
& +2 x^{2} m_{N^{*}}^{2} \widehat{\mathcal{V}}_{1} \beta-7 x^{2} m_{N^{*}}^{2} \widehat{\mathcal{V}}_{3} \beta-3 x^{2} m_{N^{*}}^{2} \widehat{\mathcal{P}}_{1} \beta \\
& +\widehat{\mathcal{V}}_{1} Q^{2} \beta-x^{2} m_{N^{*}}^{2} \widehat{\mathcal{S}}_{1} \beta-\widehat{\mathcal{A}}_{3} x^{2} m_{N^{*}}^{2}(7+5 \beta) \\
& -\widehat{\mathcal{A}}_{1}\left[Q^{2}-Q^{2} \beta+2 x^{2} m_{N^{*}}^{2}(1+2 \beta)\right] \\
& +9 x^{2} m_{N^{*}}^{2} \widehat{\mathcal{T}}_{1}-2 Q^{2} \widehat{\mathcal{T}}_{1}-9 x^{2} m_{N^{*}}^{2} \beta \widehat{\mathcal{T}}_{1} \\
& +2 Q^{2} \beta \widehat{T}_{1}+12 x^{2} m_{N^{*}}^{2}(-1+\beta) \widehat{T}_{3} \beta \\
& +m_{b}\left[x m _ { N ^ { * } } \left(-4 \widehat{\mathcal{A}}_{3}+3 \widehat{\mathcal{V}}_{1}\right.\right. \\
& -4 \widehat{\mathcal{V}}_{3}+\widehat{\mathcal{P}}_{1}+\widehat{\mathcal{S}}_{1}+\widehat{\mathcal{A}}_{1}(-1+\beta) \\
& +5 \widehat{\mathcal{T}}_{1}+\beta\left(4 \widehat{\mathcal{A}}_{3}-3 \widehat{\mathcal{V}}_{1}+4 \widehat{\mathcal{V}}_{3}\right. \\
& \left.\left.+\widehat{\mathcal{P}}_{1}+\widehat{\mathcal{S}}_{1}+9 \widehat{\mathcal{T}}_{1}-14 \widehat{\mathcal{T}}_{3}\right)-14 \widehat{\mathcal{T}}_{3}\right) \\
& \left.\left.+(-1+\beta)\left(\widehat{\mathcal{A}}_{1}+\widehat{\mathcal{V}}_{1}+2 \widehat{\mathcal{T}}_{1}\right) m_{b}\right]\right\} \\
& +\frac{m_{N^{*}}^{2}}{4 x}(-1+\beta)\left[\widehat{A}_{1}^{M}+\widehat{V}_{1}^{M}+2 \widehat{T}_{1}^{M}\right] \text {. } \\
& \begin{aligned}
\mathcal{D}_{42}^{I I}(x)= & \frac{m_{N^{*}}^{2}}{4 x}\left\{x m _ { N ^ { * } } \left(3 \widetilde{\widetilde{\mathcal{A}}}_{6} x^{2} m_{N^{*}}^{2}(1+\beta)\right.\right. \\
& -Q^{2}(-1+\beta)\left(\widetilde{\widetilde{\mathcal{T}}}_{6}-\widetilde{\widetilde{\mathcal{T}}}_{8}\right)+3 x^{2} m_{N^{*}}^{2}
\end{aligned} \\
& \left.\left(\widetilde{\widetilde{\mathcal{V}}}_{6}+\widetilde{\widetilde{\mathcal{V}}}_{6} \beta+2 \widetilde{\widetilde{\mathcal{T}}}_{8}-2 \beta \widetilde{\widetilde{\mathcal{T}}}_{8}\right)\right)+m_{b}\left[2 \widetilde{\widetilde{\mathcal{A}}}_{6} x^{2} m_{N^{*}}^{2}\right. \\
& (-1+\beta)+2 Q^{2} \beta \widetilde{\widetilde{\mathcal{T}}}_{6}-x^{2} m_{N^{*}}^{2}(-1+\beta)\left(2 \widetilde{\widetilde{\mathcal{V}}}_{6}\right. \\
& \left.+\widetilde{\widetilde{\mathcal{T}}}_{6}-4 \widetilde{\widetilde{\mathcal{T}}}_{8}\right)+m_{b}\left(3 x m _ { N ^ { * } } \left[\left(\widetilde{\widetilde{\mathcal{A}}}_{6}+\widetilde{\widetilde{V}}_{6}\right)(1+\beta)\right.\right. \\
& \left.\left.\left.\left.-2(-1+\beta) \widetilde{\widetilde{\mathcal{T}}}_{8}\right]+(-1+\beta) \widetilde{\widetilde{\mathcal{T}}}_{6} m_{b}\right)\right]\right\}+\frac{m_{N^{*}}}{8 x} \\
& \left\{-\left[( x ^ { 2 } m _ { N ^ { * } } ^ { 2 } + Q ^ { 2 } ) \left(-3 x^{2} m_{N^{*}}^{2} \tilde{\mathcal{V}}_{4}+9 x^{2} m_{N^{*}}^{2} \tilde{\mathcal{V}}_{5}\right.\right.\right. \\
& -4 x^{2} m_{N^{*}}^{2} \widetilde{\mathcal{P}}_{2}+4 x^{2} m_{N^{*}}^{2} \widetilde{\mathcal{S}}_{2}-3 x^{2} m_{N^{*}}^{2} \widetilde{\mathcal{V}}_{4} \beta \\
& +9 x^{2} m_{N^{*}}^{2} \widetilde{\mathcal{V}}_{5} \beta+4 x^{2} m_{N^{*}}^{2} \widetilde{\mathcal{P}}_{2} \beta-4 x^{2} m_{N^{*}}^{2} \widetilde{\mathcal{S}}_{2} \beta \\
& +3 \widetilde{\mathcal{A}}_{4} x^{2} m_{N^{*}}^{2}(1+\beta)-9 \widetilde{\mathcal{A}}_{5} x^{2} m_{N^{*}}^{2}(1+\beta) \\
& -2 x^{2} m_{N^{*}}^{2} \widetilde{\mathcal{T}}_{2}-Q^{2} \widetilde{\mathcal{T}}_{2}+2 x^{2} m_{N^{*}}^{2} \beta \widetilde{\mathcal{T}}_{2}+Q^{2} \beta \widetilde{\mathcal{T}}_{2} \\
& +2 x^{2} m_{N^{*}}^{2} \widetilde{\mathcal{T}}_{4}-Q^{2} \widetilde{\mathcal{T}}_{4}-2 x^{2} m_{N^{*}}^{2} \beta \widetilde{\mathcal{T}}_{4}+Q^{2} \beta \widetilde{\mathcal{T}}_{4} \\
& -12 x^{2} m_{N^{*}}^{2} \widetilde{\mathcal{T}}_{5}+12 x^{2} m_{N^{*}}^{2} \beta \widetilde{\mathcal{T}}_{5} \\
& \left.\left.+24 x^{2} m_{N^{*}}^{2}(-1+\beta) \widetilde{\mathcal{T}}_{7}\right)\right] \\
& +m_{b}\left[-\left(x m _ { N ^ { * } } \left\{-\left(\tilde{\mathcal{A}}_{5} x^{2} m_{N^{*}}^{2}\right)\right.\right.\right. \\
& +2 x^{2} m_{N^{*}}^{2} \tilde{V}_{2}-x^{2} m_{N^{*}}^{2} \tilde{V}_{4} \\
& -x^{2} m_{N^{*}}^{2} \widetilde{\mathcal{V}}_{5}+6 \widetilde{\mathcal{A}}_{5} Q^{2}+4 \widetilde{\mathcal{V}}_{2} Q^{2}+2 \widetilde{\mathcal{V}}_{4} Q^{2} \\
& -6 \widetilde{\mathcal{V}}_{5} Q^{2}+2 \widetilde{\mathcal{P}}_{2} Q^{2}-2 Q^{2} \widetilde{\mathcal{S}}_{2} \\
& \mathcal{D}_{43}^{I I}(x)=-\frac{m_{N^{*}}^{2}}{4 x}\left\{2 x m_{N^{*}}\left(x^{2} m_{N^{*}}^{2}+Q^{2}\right)^{2}(-1+\beta) \widetilde{\widetilde{\mathcal{T}}}_{6}\right. \\
& +m_{b}\left[-\left(\left(x^{2} m_{N^{*}}^{2}+Q^{2}\right)\right.\right. \\
& +2 \widetilde{\mathcal{A}}_{2}\left(x^{2} m_{N^{*}}^{2}-2 Q^{2}\right)(-1+\beta) \\
& +\widetilde{\mathcal{A}}_{4}\left(x^{2} m_{N^{*}}^{2}+2 Q^{2}\right)(-1+\beta)+\widetilde{\mathcal{A}}_{5} x^{2} m_{N^{*}}^{2} \beta \\
& -2 x^{2} m_{N^{*}}^{2} \widetilde{\mathcal{V}}_{2} \beta+x^{2} m_{N^{*}}^{2} \widetilde{\mathcal{V}}_{4} \beta+x^{2} m_{N^{*}}^{2} \widetilde{\mathcal{V}}_{5} \beta \\
& -6 \widetilde{\mathcal{A}}_{5} Q^{2} \beta-4 \widetilde{\mathcal{V}}_{2} Q^{2} \beta-2 \widetilde{\mathcal{V}}_{4} Q^{2} \beta+6 \widetilde{\mathcal{V}}_{5} Q^{2} \beta
\end{aligned}
$$




$$
\begin{aligned}
& +2 \widetilde{\mathcal{P}}_{2} Q^{2} \beta-2 Q^{2} \widetilde{\mathcal{S}}_{2} \beta+2 Q^{2} \widetilde{\mathcal{T}}_{2}+2 Q^{2} \beta \widetilde{\mathcal{T}}_{2} \\
& -4 x^{2} m_{N^{*}}^{2} \widetilde{\mathcal{T}}_{4}+12 Q^{2} \widetilde{\mathcal{T}}_{4}+4 x^{2} m_{N^{*}}^{2} \beta \widetilde{\mathcal{T}}_{4} \\
& +12 Q^{2} \beta \widetilde{\mathcal{T}}_{4}+4 x^{2} m_{N^{*}}^{2} \widetilde{\mathcal{T}}_{5}+14 Q^{2} \widetilde{\mathcal{T}}_{5} \\
& \left.\left.-4 x^{2} m_{N^{*}}^{2} \beta \widetilde{\mathcal{T}}_{5}+14 Q^{2} \beta \widetilde{\mathcal{T}}_{5}+28 Q^{2}(1+\beta) \widetilde{\mathcal{T}}_{7}\right\}\right) \\
& +m_{b}\left(-9 \widetilde{\mathcal{A}}_{5} x^{2} m_{N^{*}}^{2}-6 x^{2} m_{N^{*}}^{2} \tilde{\mathcal{V}}_{2}\right. \\
& -3 x^{2} m_{N^{*}}^{2} \widetilde{\mathcal{V}}_{4}+9 x^{2} m_{N^{*}}^{2} \tilde{\mathcal{V}}_{5}-4 x^{2} m_{N^{*}}^{2} \widetilde{\mathcal{P}}_{2}+6 \tilde{\mathcal{V}}_{2} Q^{2} \\
& +4 x^{2} m_{N^{*}}^{2} \widetilde{\mathcal{S}}_{2}-9 \widetilde{\mathcal{A}}_{5} x^{2} m_{N^{*}}^{2} \beta-6 x^{2} m_{N^{*}}^{2} \widetilde{\mathcal{V}}_{2} \beta \\
& -3 x^{2} m_{N^{*}}^{2} \widetilde{\mathcal{V}}_{4} \beta+9 x^{2} m_{N^{*}}^{2} \widetilde{\mathcal{V}}_{5} \beta \\
& +4 x^{2} m_{N^{*}}^{2} \widetilde{\mathcal{P}}_{2} \beta+6 \widetilde{\mathcal{V}}_{2} Q^{2} \beta-4 x^{2} m_{N^{*}}^{2} \widetilde{\mathcal{S}}_{2} \beta \\
& +3 \widetilde{\mathcal{A}}_{4} x^{2} m_{N^{*}}^{2}(1+\beta) \\
& -6 \widetilde{\mathcal{A}}_{2}\left(x^{2} m_{N^{*}}^{2}-Q^{2}\right)(1+\beta)-4 x^{2} m_{N^{*}}^{2} \widetilde{\mathcal{T}}_{2} \\
& +3 Q^{2} \widetilde{\mathcal{T}}_{2}+4 x^{2} m_{N^{*}}^{2} \beta \widetilde{\mathcal{T}}_{2}-3 Q^{2} \beta \widetilde{\mathcal{T}}_{2} \\
& -8 x^{2} m_{N^{*}}^{2} \widetilde{\mathcal{T}}_{4} \\
& +11 Q^{2} \widetilde{\mathcal{T}}_{4}+8 x^{2} m_{N^{*}}^{2} \beta \widetilde{\mathcal{T}}_{4}-11 Q^{2} \beta \widetilde{\mathcal{T}}_{4} \\
& -12 x^{2} m_{N^{*}}^{2} \widetilde{\mathcal{T}}_{5}+12 x^{2} m_{N^{*}}^{2} \beta \widetilde{\mathcal{T}}_{5} \\
& +24 x^{2} m_{N^{*}}^{2}(-1+\beta) \widetilde{\mathcal{T}}_{7} \\
& +m_{b}\left\{x m _ { N ^ { * } } ( - 1 + \beta ) \left(2 \widetilde{\mathcal{A}}_{2}+\widetilde{\mathcal{A}}_{4}\right.\right. \\
& +\widetilde{\mathcal{A}}_{5}-2 \tilde{\mathcal{V}}_{2}+\tilde{\mathcal{V}}_{4}+\tilde{\mathcal{V}}_{5} \\
& \left.+4 \widetilde{\mathcal{T}}_{4}-4 \widetilde{\mathcal{T}}_{5}\right)+2\left[3 \widetilde{\mathcal{A}}_{2}(1+\beta)\right. \\
& \left.\left.\left.\left.\left.+3 \widetilde{\mathcal{V}}_{2}(1+\beta)-(-1+\beta)\left(\widetilde{\mathcal{T}}_{2}+5 \widetilde{\mathcal{T}}_{4}\right]\right) m_{b}\right\}\right)\right]\right\} \\
& +\frac{m_{N^{*}}^{2}}{4 x}\left\{-3 x^{3} m_{N^{*}}^{3}\left(\widehat{A}_{1}^{M}-\widehat{V}_{1}^{M}\right)(1+\beta)\right. \\
& -x m_{N^{*}}\left(6 x^{2} m_{N^{*}}^{2}-Q^{2}\right)(-1+\beta) \widehat{T}_{1}^{M} \\
& +3 x m_{N^{*}}\left[\left(-\widehat{A}_{1}^{M}+\widehat{V}_{1}^{M}\right)(1+\beta)\right. \\
& \left.-2(-1+\beta) \widehat{T}_{1}^{M}\right] m_{b}^{2} \\
& \left.-2(-1+\beta)\left(\widehat{A}_{1}^{M}+\widehat{V}_{1}^{M}-2 \widehat{T}_{1}^{M}\right) m_{b}^{3}\right\} . \\
& \mathcal{D}_{41}^{I I}(x)=\frac{m_{N^{*}}^{2}}{4 x}\left\{x m _ { N ^ { * } } \left[3 \widetilde{\widetilde{\mathcal{A}}}_{6}(1+\beta)+3 \widetilde{\widetilde{\mathcal{V}}}_{6}(1+\beta)\right.\right. \\
& \left.+2(-1+\beta)\left(\widetilde{\widetilde{\mathcal{T}}}_{6}-3 \widetilde{\widetilde{\mathcal{T}}}_{8}\right)\right] \\
& \left.+\left(\widetilde{\widetilde{\mathcal{T}}}_{6}-t \widetilde{\widetilde{\mathcal{T}}}_{6}\right) m_{b}\right\}+\frac{m_{N^{*}}}{8 x}\left\{3 x^{2} m_{N^{*}}^{2} \tilde{\mathcal{V}}_{4}\right. \\
& -9 x^{2} m_{N^{*}}^{2} \widetilde{\mathcal{V}}_{5}+4 x^{2} m_{N^{*}}^{2} \widetilde{\mathcal{P}}_{2}-4 x^{2} m_{N^{*}}^{2} \widetilde{\mathcal{S}}_{2} \\
& +3 x^{2} m_{N^{*}}^{2} \widetilde{\mathcal{V}}_{4} \beta-9 x^{2} m_{N^{*}}^{2} \widetilde{\mathcal{V}}_{5} \beta \\
& -4 x^{2} m_{N^{*}}^{2} \widetilde{\mathcal{P}}_{2} \beta+4 x^{2} m_{N^{*}}^{2} \widetilde{\mathcal{S}}_{2} \beta \\
& -3 \widetilde{\mathcal{A}}_{4} x^{2} m_{N^{*}}^{2}(1+\beta) \\
& +9 \widetilde{\mathcal{A}}_{5} x^{2} m_{N^{*}}^{2}(1+\beta) \\
& +2 x^{2} m_{N^{*}}^{2} \widetilde{\mathcal{T}}_{2}-Q^{2} \widetilde{\mathcal{T}}_{2}-2 x^{2} m_{N^{*}}^{2} \beta \widetilde{\mathcal{T}}_{2} \\
& +Q^{2} \beta \widetilde{\mathcal{T}}_{2}-2 x^{2} m_{N^{*}}^{2} \widetilde{\mathcal{T}}_{4} \\
& -Q^{2} \widetilde{\mathcal{T}}_{4}+2 x^{2} m_{N^{*}}^{2} \beta \widetilde{\mathcal{T}}_{4}+Q^{2} \beta \widetilde{\mathcal{T}}_{4}+12 x^{2} m_{N^{*}}^{2} \widetilde{\mathcal{T}}_{5} \\
& -12 x^{2} m_{N^{*}}^{2} \beta \widetilde{\mathcal{T}}_{5}-24 x^{2} m_{N^{*}}^{2}(-1+\beta) \widetilde{\mathcal{T}}_{7}
\end{aligned}
$$

$$
\begin{aligned}
& +m_{b}\left[-\left(x m _ { N ^ { * } } ( - 1 + \beta ) \left[2 \widetilde{\mathcal{A}}_{2}+\widetilde{\mathcal{A}}_{4}+\widetilde{\mathcal{A}}_{5}\right.\right.\right. \\
& \left.\left.-2 \tilde{\mathcal{V}}_{2}+\tilde{\mathcal{V}}_{4}+\tilde{\mathcal{V}}_{5}+4 \widetilde{\mathcal{T}}_{4}-4 \widetilde{\mathcal{T}}_{5}\right]\right) \\
& -2\left(3 \widetilde{\mathcal{A}}_{2}(1+\beta)+3 \widetilde{\mathcal{V}}_{2}(1+\beta)\right. \\
& \left.\left.\left.-(-1+\beta)\left(\widetilde{\mathcal{T}}_{2}+5 \widetilde{\mathcal{T}}_{4}\right)\right) m_{b}\right]\right\} \\
& +\frac{1}{4 x}\left\{x m _ { N ^ { * } } \left[-3 x^{2} m_{N^{*}}^{2} \widehat{\mathcal{V}}_{1}+6 x^{2} m_{N^{*}}^{2} \widehat{\mathcal{V}}_{3}\right.\right. \\
& -2 x^{2} m_{N^{*}}^{2} \widehat{\mathcal{P}}_{1}+\widehat{\mathcal{V}}_{3} Q^{2}+\widehat{\mathcal{P}}_{1} Q^{2}-2 x^{2} m_{N^{*}}^{2} \widehat{\mathcal{S}}_{1} \\
& -Q^{2} \widehat{\mathcal{S}}_{1}-3 x^{2} m_{N^{*}}^{2} \widehat{\mathcal{V}}_{1} \beta+6 x^{2} m_{N^{*}}^{2} \widehat{\mathcal{V}}_{3} \beta \\
& +2 x^{2} m_{N^{*}}^{2} \widehat{\mathcal{P}}_{1} \beta-\widehat{\mathcal{V}}_{3} Q^{2} \beta \\
& -\widehat{\mathcal{P}}_{1} Q^{2} \beta+2 x^{2} m_{N^{*}}^{2} \widehat{\mathcal{S}}_{1} \beta \\
& +Q^{2} \widehat{\mathcal{S}}_{1} \beta+3 \widehat{\mathcal{A}}_{1} x^{2} m_{N^{*}}^{2}(1+\beta) \\
& +\widehat{\mathcal{A}}_{3}\left[Q^{2}(-1+\beta)+6 x^{2} m_{N^{*}}^{2}(1+\beta)\right] \\
& -6 x^{2} m_{N^{*}}^{2} \widehat{\mathcal{T}}_{1} \\
& +Q^{2} \widehat{\mathcal{T}}_{1}+6 x^{2} m_{N^{*}}^{2} \beta \widehat{\mathcal{T}}_{1}-Q^{2} \beta \widehat{\mathcal{T}}_{1} \\
& \left.-12 x^{2} m_{N^{*}}^{2}(-1+\beta) \widehat{\mathcal{T}}_{3}\right] \\
& +\left(x^{2} m_{N^{*}}^{2}-Q^{2}\right)(-1+\beta)\left(\widehat{\mathcal{A}}_{1}+\widehat{\mathcal{V}}_{1}\right. \\
& \left.-2 \widehat{\mathcal{T}}_{1}\right) m_{b}-x m_{N^{*}}\left[-3 \widehat{\mathcal{V}}_{1}+6 \widehat{\mathcal{V}}_{3}+3 \widehat{\mathcal{A}}_{1}(1+\beta)\right. \\
& +6 \widehat{\mathcal{A}}_{3}(1+\beta)+\beta\left(-3 \widehat{\mathcal{V}}_{1}+2\left[3 \widehat{\mathcal{V}}_{3}\right.\right. \\
& \left.\left.+\widehat{\mathcal{P}}_{1}+\widehat{\mathcal{S}}_{1}+3 \widehat{\mathcal{T}}_{1}-6 \widehat{\mathcal{T}}_{3}\right]\right) \\
& \left.-2\left(\widehat{\mathcal{P}}_{1}+\widehat{\mathcal{S}}_{1}+3 \widehat{\mathcal{T}}_{1}-6 \widehat{\mathcal{T}}_{3}\right)\right] m_{b}^{2} \\
& \left.-(-1+\beta)\left(\widehat{\mathcal{A}}_{1}+\widehat{\mathcal{V}}_{1}-2 \widehat{\mathcal{T}}_{1}\right) m_{b}^{3}\right\} \\
& +\frac{3 m_{N^{*}}^{3}}{4}\left[\left(-\widehat{A}_{1}^{M}+\widehat{V}_{1}^{M}\right)(1+\beta)-2(-1+\beta) \widehat{T}_{1}^{M}\right] .
\end{aligned}
$$

\section{Appendix C: Differential widths for the $\Lambda_{b} \rightarrow N^{*} \ell^{+} \ell^{-}$ decays}

In this Appendix we present the differential widths for the $\Lambda_{b} \rightarrow N^{*} \ell^{+} \ell^{-},(\ell=e, \mu, \tau)$ decays. After lengthy, but straightforward calculations, for the differential rate of the $\Lambda_{b} \rightarrow N^{*} \ell^{+} \ell^{-}$we get,

$$
\frac{d \Gamma(s)}{d s}=\frac{G^{2} \alpha_{e m}^{2} m_{\Lambda_{b}}}{4096 \pi^{5}}\left|V_{t b} V_{t d}^{*}\right|^{2} v \sqrt{\lambda(1, r, s)}\left[T_{1}(s)+\frac{1}{3} T_{2}(s)\right],
$$

where $s=q^{2} / m_{\Lambda_{b}}^{2}, r=m_{N^{*}}^{2} / m_{\Lambda_{b}}^{2}, G_{F}=1.17 \times 10^{-5}$ $\mathrm{GeV}^{-2}$ is the Fermi coupling constant, $v=\sqrt{1-4 m_{\ell}^{2} / q^{2}}$ is the lepton velocity, and $\lambda(a, b, c)=a^{2}+b^{2}+c^{2}-2 a b-$ $2 a c-2 b c$ is the usual triangle function. For the element of the CKM matrix $\left|V_{t b} V_{t d}^{*}\right|=(8.2 \pm 0.6) \times 10^{-3}$ has been used [13]. The functions $T_{1}(s)$ and $T_{2}(s)$ are given as: 


$$
\begin{aligned}
& T_{1}(s)=8 m_{\Lambda_{b}}^{2}\{(1+2 \sqrt{r}+r-s) \\
& {\left[4 m_{\ell}^{2}+m_{\Lambda_{b}}^{2}(1-2 \sqrt{r}+r+s)\right]\left|F_{1}\right|^{2}} \\
& -\left[4 m_{\ell}^{2}(1+6 \sqrt{r}+r-s)-m_{\Lambda_{b}}^{2}\left((1-r)^{2}\right.\right. \\
& \left.\left.+4 \sqrt{r} s-s^{2}\right)\right]\left|F_{4}\right|^{2} \\
& +(1+2 \sqrt{r}+r-s)\left[4 m_{\ell}^{2}(1-\sqrt{r})^{2}\right. \\
& \left.+m_{\Lambda_{b}}^{2} s(1-2 \sqrt{r}+r+s)\right]\left|F_{2}\right|^{2} \\
& +m_{\Lambda_{b}}^{2} s\left[(1-r)^{2}+4 \sqrt{r} s-s^{2}\right] v^{2}\left|F_{4}\right|^{2} \\
& +4 m_{\ell}^{2}(1-2 \sqrt{r}+r-s) s\left|F_{6}\right|^{2} \\
& +(1-2 \sqrt{r}+r-s) \\
& {\left[4 m_{\ell}^{2}+m_{\Lambda_{b}}^{2}(1+2 \sqrt{r}+r+s)\right]\left|G_{1}\right|^{2}} \\
& -\left[4 m_{\ell}^{2}(1-6 \sqrt{r}+r-s)-m_{\Lambda_{b}}^{2}\left((1-r)^{2}\right.\right. \\
& \left.\left.-4 \sqrt{r} s-s^{2}\right)\right]\left|G_{4}\right|^{2} \\
& +(1-2 \sqrt{r}+r-s)\left[4 m_{\ell}^{2}(1+\sqrt{r})^{2}\right. \\
& \left.+m_{\Lambda_{b}}^{2} s(1+2 \sqrt{r}+r+s)\right]\left|G_{2}\right|^{2} \\
& +m_{\Lambda_{b}}^{2} s\left[(1-r)^{2}-4 \sqrt{r} s-s^{2}\right] v^{2}\left|G_{5}\right|^{2} \\
& +4 m_{\ell}^{2}(1+2 \sqrt{r}+r-s) s\left|G_{6}\right|^{2} \\
& -4(1-\sqrt{r})(1+2 \sqrt{r}+r-s)\left(2 m_{\ell}^{2}\right. \\
& \left.+m_{\Lambda_{b}}^{2} s\right) \operatorname{Re}\left[F_{1}^{*} F_{2}\right] \\
& -4 m_{\Lambda_{b}}^{2}(1-\sqrt{r})(1+2 \sqrt{r}+r-s) s v^{2} \operatorname{Re}\left[F_{4}^{*} F_{5}\right] \\
& -8 m_{\ell}^{2}(1+\sqrt{r})(1-2 \sqrt{r}+r-s) \operatorname{Re}\left[F_{4}^{*} F_{6}\right] \\
& -4(1+\sqrt{r})(1-2 \sqrt{r}+r-s)\left(2 m_{\ell}^{2}\right. \\
& \left.+m_{\Lambda_{b}}^{2} s\right) \operatorname{Re}\left[G_{1}^{*} G_{2}\right] \\
& \left.-4 m_{\Lambda_{b}}^{2}(1+\sqrt{r})(1-2 \sqrt{r}+r-s) s v^{2} \operatorname{Re}\left[G_{4}^{*} G_{5}\right]\right] \\
& \left.-8 m_{\ell}^{2}(1-\sqrt{r})(1+2 \sqrt{r}+r-s) \operatorname{Re}\left[G_{4}^{*} G_{6}\right]\right\}, \\
& T_{2}(s)=-8 m_{\Lambda_{b}}^{4} v^{2} \lambda(1, r, s) \\
& {\left[\left|F_{1}\right|^{2}+\left|F_{4}\right|^{2}+\left|G_{1}\right|^{2}+\left|G_{4}\right|^{2}\right.} \\
& \left.-s\left(\left|F_{2}\right|^{2}+\left|F_{5}\right|^{2}+\left|G_{2}\right|^{2}+\left|G_{5}\right|^{2}\right)\right] \text {. }
\end{aligned}
$$

The differential decay width for the $\Lambda_{b}^{*} \rightarrow N^{*} \ell^{+} \ell^{-}$transition cam be obtained from the differential decay width for the $\Lambda_{b} \rightarrow N^{*} \ell^{+} \ell^{-}$by making the following replacements: $F_{i} \rightarrow \widetilde{G}_{i}, G_{i} \rightarrow \widetilde{F}_{i}, m_{N} \rightarrow-m_{N}$, and by changing the sign in front of the terms $\operatorname{Re}\left[F_{4}^{*} F_{5}\right], \operatorname{Re}\left[F_{4}^{*} F_{6}\right]$, and $\operatorname{Re}\left[G_{4}^{*} G_{5}\right]$, as well as $m_{\Lambda_{b}} \rightarrow m_{\Lambda_{b}^{*}}, s \rightarrow s^{\prime}=q^{2} / m_{\Lambda_{b}^{*}}^{2}$, and $r \rightarrow r^{\prime}=m_{N^{*}}^{2} / m_{\Lambda_{b}^{*}}^{2}$. where

$$
\begin{aligned}
& F_{1}\left(q^{2}\right)=c_{9} g_{1}\left(q^{2}\right)-\frac{2 m_{b}}{m_{\Lambda_{b}^{*}}} c_{7} g_{1}^{T}\left(q^{2}\right) \\
& F_{2}\left(q^{2}\right)=-c_{9} g_{2}\left(q^{2}\right)-\frac{2 m_{b}}{q^{2}} m_{\Lambda_{b}^{*}} c_{7} g_{2}^{T}\left(q^{2}\right), \\
& F_{3}\left(q^{2}\right)=-c_{9} g_{3}\left(q^{2}\right)-\frac{m_{b}}{q^{2}}\left(m_{\Lambda_{b}^{*}}-m_{N}\right) c_{7} g_{1}^{T}\left(q^{2}\right), \\
& G_{1}\left(q^{2}\right)=c_{9} f_{1}\left(q^{2}\right)-\frac{2 m_{b}}{m_{\Lambda_{b}^{*}}} c_{7} f_{1}^{T}\left(q^{2}\right), \\
& G_{2}\left(q^{2}\right)=-c_{9} f_{2}\left(q^{2}\right)-\frac{2 m_{b}}{q^{2}} m_{\Lambda_{b}^{*}} c_{7} f_{2}^{T}\left(q^{2}\right), \\
& G_{3}\left(q^{2}\right)=-c_{9} f_{3}\left(q^{2}\right)-\frac{2 m_{b}}{q^{2}}\left(m_{\Lambda_{b}^{*}}+m_{N}\right) c_{7} f_{1}^{T}\left(q^{2}\right), \\
& F_{4}\left(q^{2}\right)=c_{10} g_{1}\left(q^{2}\right), \\
& F_{5}\left(q^{2}\right)=-c_{10} g_{2}\left(q^{2}\right), \\
& F_{6}\left(q^{2}\right)=-c_{10} g_{3}\left(q^{2}\right), \\
& G_{4}\left(q^{2}\right)=c_{10} f_{1}\left(q^{2}\right), \\
& G_{5}\left(q^{2}\right)=-c_{10} f_{2}\left(q^{2}\right), \\
& G_{6}\left(q^{2}\right)=-c_{10} f_{3}\left(q^{2}\right) .
\end{aligned}
$$

\section{References}

1. T. Aaltonen et al., CDF Collaboration, Phys. Rev. Lett. 107, 201802 (2011)

2. R. Aaij et al., LHCb Collaboration, Phys. Lett. B 725, 25 (2013)

3. R. Aaij et al., LHCb Collaboration, JHEP 1506, 115 (2015)

4. R. Aaij et al., LHCb Collaboration, JHEP 1704, 029 (2017)

5. T.M. Aliev, T. Barakat, M. Savc1, Phys. Rev. D 98, 035333 (2018)

6. I.I. Balitsky, V.M. Braun, A.V. Kolesnichenko, Nucl. Phys. B 312 , 509 (1989)

7. V.L. Chernyak, I.R. Zhitnitsky, Nucl. Phys. B 345, 137 (1990)

8. M.A. Shifman, A.I. Vainshtein, V.I. Zakharov, Nucl. Phys. B 147, 385 (1979)

9. I.G. Aznauryan et al., Int. J. Mod. Phys. E 22, 1330015 (2015)

10. M. Emmerich, N. Offen, A. Schafer, J. Phys. G 43, 115003 (2016)

11. C.H. Chen, C.Q. Geng, Phys. Rev. D 64, 074001 (2001)

12. M. Emmerich, $\mathrm{PhD}$ thesis, Regensburg (2017)

13. T. Mannel, Y.-M. Wang, JHEP 1112, 067 (2011)

14. T. Feldmann, M.W.Y. Yip, Phys. Rev. D 85, 014035 (2012). Erratum: Phys. Rev. D 86, 079901 (2012)

15. Y.-M. Wang, Y.-L. Shen, JHEP 1602, 179 (2016)

16. Y.M. Wang, J Conf Ser 556, 012050 (2014)

17. M. Tanabashi et al., Particle Data Group, Phys. Rev. D 98, 030001 (2018)

18. V.M. Braun, A. Lenz, N. Mahnke, E. Stein, Phys. Rev. D 65, 074011 (2002)

19. V.M. Braun, R.J. Fries, N. Mahnke, E. Stein, Nucl. Phys. B 589, $381(2000)$

20. V.M. Braun, R.J. Fries, N. Mahnke, E. Stein, Nucl. Phys. B 607, $433(2001)$

21. I.V. Anikin, V.M. Braun, N. Offen, Phys. Rev. D 88, 114021 (2013)

22. I.V. Anikin, V.M. Braun, N. Offen, Phys. Rev. D 92, 014018 (2015)

23. V.M. Braun et al., Phys. Rev. D 89, 094511 (2014)

24. C. Bourrely, I. Caprini, L. Lellouch, Phys. Rev. D 79, 013008 (2009) 